Research Article

\title{
Solutions of a Class of Degenerate Kinetic Equations Using Steepest Descent in Wasserstein Space
}

\author{
Aboubacar Marcos $(\mathbb{D}$ and Ambroise Soglo \\ Institut de Mathématiques et de Sciences Physiques, Université d'Abomey-Calavi, Abomey-Calavi, Benin \\ Correspondence should be addressed to Aboubacar Marcos; abmarcos@imsp-uac.org \\ Received 16 January 2020; Accepted 9 April 2020; Published 9 June 2020 \\ Academic Editor: Yongqiang Fu \\ Copyright ( 2020 Aboubacar Marcos and Ambroise Soglo. This is an open access article distributed under the Creative Commons \\ Attribution License, which permits unrestricted use, distribution, and reproduction in any medium, provided the original work is \\ properly cited. \\ We use the steepest descent method in an Orlicz-Wasserstein space to study the existence of solutions for a very broad class of \\ kinetic equations, which include the Boltzmann equation, the Vlasov-Poisson equation, the porous medium equation, and the \\ parabolic $p$-Laplacian equation, among others. We combine a splitting technique along with an iterative variational scheme to \\ build a discrete solution which converges to a weak solution of our problem.
}

\section{Introduction}

The general model describing the kinetic equations is about an evolution equation of unknown function $f$, representing a time-depending density of probability distribution of a material in a given domain of the space. In the present work, $f$ may measure the density distribution of a system of identical particles of a bulk material. The density $f$ depends on the time $t$ and the position $x$ and the velocity $v$ of some particles at $t$. Roughly speaking, the equation is considered as the evolution of the density function in the phase space $\Omega \times \mathbb{R}^{d}$, with $\Omega$ as an open bounded domain with periodic boundary. As a probability density, $f$ remains positive in the court of time and satisfies the mass conservation principle: $\int_{\Omega \times \mathbb{R}^{d}} f(t, x, v) \mathrm{d} x \mathrm{~d} v=1$, for all $t \geq 0$ :

$$
\left\{\begin{array}{l}
\frac{\partial f(t, x, v)}{\partial t}+v \cdot \nabla_{x} f(t, x, v)=\operatorname{div}_{v}\left\{f(t, x, v) \nabla c^{*}\left[\nabla_{v}\left(G^{\prime}(f(t, x, v))\right)\right]\right\}, \quad \text { in }[0, \infty) \times \Omega \times \mathbb{R}^{d}, \\
f(0, x, v)=f_{0}(x, v), \quad \text { in } \Omega \times \mathbb{R}^{d},
\end{array}\right.
$$

$$
\begin{aligned}
& \frac{\partial f(t, x, v)}{\partial t}+v \cdot \nabla_{x} f(t, x, v)=0, \\
& \frac{\partial f(t, x, v)}{\partial t}-\operatorname{div}_{v}\left\{f(t, x, v) \nabla c^{*}\left[\nabla_{v}\left(G^{\prime}(f(t, x, v))\right)\right]=0 .\right.
\end{aligned}
$$

$\Omega \times \mathbb{R}^{d}$. Here, $\Omega$ is an open, bounded, convex, and smooth domain of $\mathbb{R}^{d},(d \geq 1)$ with $\partial \Omega$ periodic, $c^{*}$ is the Legendre transform of a cost function $c$, and $G:[0, \infty) \longrightarrow \mathbb{R}$ is a convex function.

Equation (1) can be viewed as a balance result of a streaming phenomenon with a general nonlinear interaction phenomenon between the particles described, respectively, as
Accordingly, the transport equation (2) can somewhat be interpreted as a relaxation of (1) at the absence of the 
interaction phenomena, whereas it reduced to (3) in absence of streaming.

One of the interests in considering (1) under a general nonlinearity is that it covers a very broad range of problems which occurred in physics and it is a purely mathematical challenge. Of course, it has been motivated by some previous works in the literature, namely, the works in [1-7], where (1) is investigated in some particular cases. Indeed, in [3], the authors dealt with the heat equation:

$$
\frac{\partial \rho(t, v)}{\partial t}=\Delta_{v} \rho(t, v), \quad \text { in }[0, \infty) \times \mathbb{R}^{d} .
$$

By fixing an probability density $\rho_{0}$ with $\int_{\mathbb{R}^{d}}|v|^{2} \rho_{0}(v) \mathrm{d} v$ finite and a time step $h>0$, they define the mass density $\rho_{k}^{h}$ as a discrete solution of (4) at time $t_{k}=h k$, which minimizes the functional

$$
I(\rho):=\int_{\mathbb{R}^{d}} \rho \ln \rho d v+\frac{1}{h} W_{2}^{2}\left(\rho, \rho_{k-1}^{h}\right),
$$

on $P_{2}\left(\mathbb{R}^{d}\right)$, where $P_{2}\left(\mathbb{R}^{d}\right)$ is the set of all probability density on $\mathbb{R}^{d}$ having finite second moments and $W_{2}$ is the 2Wasserstein metric defined as

$$
\begin{aligned}
& W_{2}: P_{2}\left(\mathbb{R}^{d}\right) \times P_{2}\left(\mathbb{R}^{d}\right) \longrightarrow \mathbb{R}, \\
& \left(\rho_{1}, \rho_{2}\right) \longmapsto \inf _{T_{\#} \rho_{1}=\rho_{2}}\left(\int_{\mathbb{R}^{d}}|v-T(v)|^{2} \rho_{1}(v) \mathrm{d} v\right)^{1 / 2} .
\end{aligned}
$$

By defining $\rho^{h}$ as follows: $\left\{\rho^{h}(t, v)=\rho_{k}^{h}(v)\right.$, if $t$ $\left.\in[h k, h(k+1)), \rho^{h}(0, v)=\rho_{0}(v)\right\}$, they tend $h$ to 0 and then show that the sequence $\left(\rho^{h}\right)_{h}$ converges to a nonnegative function $\rho$, which solves (4) in a weak sense.

In [1], the existence of solutions for the spatially homogeneous equations associated with (1), that is, the equation for fixed $x$

$$
\frac{\partial f}{\partial t}=\operatorname{div}_{v}\left\{f \nabla c^{*}\left(\nabla_{v}\left(G^{\prime}(f)\right)\right)\right\}, \quad \text { in }[0, \infty) \times \Omega^{\prime}, f=f(t, v),
$$

has been proved by M. Agueh, using a similar variational scheme as in [6]. Here, $\Omega^{\prime}$ is an bounded and convex domain (see [6] for more details).

A particular case of (1), namely, the kinetic equation

$$
\frac{\partial f(t, x, v)}{\partial t}+v \cdot \nabla_{x} f(t, x, v)=\Delta_{v} f(t, x, v)
$$

obtained by choosing $c^{*}(x)=|x|^{2} / 2$ and $G(x)=x \ln x$, has been studied in [5] by using a discretization scheme basing on the "splitting method." This enables the authors to decompose a discrete solution $f_{k}$ of the kinetic equation (8) in the form $f_{k}=\rho_{k}^{h} F_{k}^{x h}$, where $\rho_{k}^{h}$ stands for a discrete solution of the free transport equation

$$
\frac{\partial f(t, x, v)}{\partial t}+v \cdot \nabla_{x} f(t, x, v)=0
$$

when $v$ is fixed and $F_{k}^{x h}$ a discrete solution of the diffusion equation in (8) when $x$ is fixed.

Defining $f^{h}$ as

$$
\begin{aligned}
& f^{h}(t, x, v)=f_{k}\left(\left(x-\left(t-t_{k}\right) v, v\right), \quad \text { if } t \in[k h,(k+1) h),\right. \\
& f^{h}(0, x, v)=f_{0}(x, v), \quad \text { with } t_{k}=k h,
\end{aligned}
$$

they show that $\left(f^{h}\right)_{h}$ converges to a nonnegative function $f$ which solves the kinetic equation (8) in a weak sense.

Such a decomposition is not suitable in the case of problem (1) because of its nonlinear structure. To deal with the more general class of kinetic equation (1), we combine some ideas from the splitting method in [5] along with some techniques developed in [1] for the spatially homogeneous equations:

$$
\frac{\partial \rho(t, v)}{\partial t}=\operatorname{div}_{v}\left(\rho \nabla c^{*}\left(\nabla_{v} G^{\prime}(\rho)\right)\right), \quad \text { in }[0, \infty) \times \mathbb{R}^{d}
$$

For the best of our knowledge, our technique is new and is stated in a more general setting. It is worth mentioning that the class of the kinetic equation (1) also includes the Vlasov-Poisson equation

$$
\frac{\partial f(t, x, v)}{\partial t}+v \cdot \nabla_{x} f(t, x, v)=\operatorname{div}_{v}(\omega f(t, x, v)),
$$

obtained when

$$
c^{*}(x)=\sum_{i=1}^{d} x_{i}, \quad \omega=(1,1, \ldots, 1) \in \mathbb{R}^{d},
$$

and the parabolic $p$-Laplacian equation

$$
\frac{\partial f(t, x, v)}{\partial t}+v \cdot \nabla_{x} f(t, x, v)=\operatorname{div}_{v}\left\{|\nabla f|^{p-2} \nabla f\right\},
$$

in the case $c^{*}(x)=|x|^{p} / p$ and $G(x)=x^{\gamma} / \gamma(\gamma-1)$ with $\gamma=(2 p-3 / p-1)$.

In order to facilitate the reading of the paper, we summarize below the main steps and technical schemes according to which ours results will be carried out:

(1) First of all, we fix a time step $h>0$ and define $f_{k}$ as a discrete solution of the kinetic equation (1) at time $t_{k}=h k$, for $k \in \mathbb{N}$ (see Section 2.1).

(2) Next, we prove that the solution $T_{k}$ of the Monge problem

$$
\text { (M): } \inf _{S_{\#} F_{k}^{x h}=G_{k}^{x h}}\left\{\int_{\mathbb{R}^{d}} c\left(\frac{v-S(v)}{h}\right) F_{k}^{x h} \mathrm{~d} v\right\},
$$

is defined by

$$
T_{k}(v):=v+h \nabla c^{*}\left(\nabla_{v} G^{\prime}\left(\rho_{k} F_{k}^{x h}\right)\right)
$$

where $F_{k}^{x h}$ and $G_{k}^{x h}$ are as in Section 2.1. We use (16) to show that the sequence $\left(f_{k}\right)_{k}$ satisfies the time-discretization equation of the kinetic equation (1) 
$\frac{f_{k}-f_{k-1}}{h}+v \cdot \nabla_{x} f_{k-1}=d i v_{v}\left(f_{k} \nabla c^{*}\left(\nabla_{v} G^{\prime}\left(f_{k}\right)\right)\right)+A_{k}^{h}$,

weakly, for $k \in \mathbb{N}$, where $A_{k}^{h}$ tends to 0 and when $h$ tends to 0 .

(3) Then, we define an approximate solution $f^{h}$ of the kinetic equation (1) (see (118)), and we prove that the sequence $\left(f^{h}\right)_{h}$ converges to a nonnegative function $f$ which solves the kinetic equation (1) in a weak sense when $h$ tends to 0 .

The convergence result has been achieved as follows:

(a) The weak convergence of $\left(f^{h}\right)_{h}$ to $f$ in $L^{p}([0, T] \times$ $\left.\Omega \times \mathbb{R}^{d}\right)$ for $0<T<\infty$ follows from the displacement convexity of the functional $H(F)=\int_{\mathbb{R}^{d}}|F|^{p} \mathrm{~d} v$ on the set of all probability density (see Proposition 3 ), and its strong convergence in $L^{p}\left([0, T] \times \Omega \times \mathbb{R}^{d}\right)$ is obtained, thanks to a diagonal method combined with a result obtained in [1].

(b) The convexity of $c^{*}$ and the boundedness of $\left(\nabla_{v}\left(G^{\prime}\left(f^{h}\right)\right)\right)_{h}$ in $L^{p}\left([0, T] \times \Omega \times \mathbb{R}^{d}\right)$ help to prove a weak convergence of the nonlinear term $\left(\operatorname{div}_{v}\left(f^{\nabla} c^{*}\left(\nabla_{v} G^{\prime}\left(f^{h}\right)\right)\right)\right)_{h}$ to $\operatorname{div}_{v}\left(f \nabla c^{*}\left(\nabla_{v} G^{\prime}(f)\right)\right)$ in $\left[C_{c}^{\infty}\left([0, T] \times \Omega \times \mathbb{R}^{d}\right)\right]^{\prime}$.

(c) Finally, the strong convergence of $\left(f^{h}\right)_{h}$ to $f$ in $L^{p}\left([0, T] \times \Omega \times \mathbb{R}^{d}\right)$ and the weak convergence of the nonlinear term $\left(\operatorname{div}_{v}\left(f^{h} \nabla c^{*}\left(\nabla_{v} G^{\prime}\left(f^{h}\right)\right)\right)\right)_{h}$ to $\operatorname{div}_{v}\left(f \nabla c^{*}\left(\nabla_{v} G^{\prime}(f)\right)\right)$ in $\left[C_{c}^{\infty}\left([0, T] \times \Omega \times \mathbb{R}^{d}\right)\right]^{\prime}$ enable us to establish that $f$ is a weak solution of the kinetic equation (1).

The paper is structured as follows. In Section 2, we state the required hypotheses and set some tools relevant for our problem. In Section 3, we set the variational formulation of the discrete problem related to our problem and construct the discrete solution. Section 4 concludes our main result by proving the convergence of the discrete problem to the considered problem. Section 5 ends the paper by giving an illustration example followed by an appendix on some regularity results.

\section{Preliminaries}

Throughout this work, we will assume the following:

$\left(H_{G}\right) G:[0, \infty) \longrightarrow \mathbb{R}$ is a convex function of class $C^{2}((0, \infty))$ such that $t \longrightarrow t^{d} G\left(t^{-d}\right)$ is convex.

$\left(H_{c}\right) c \in C^{1}\left(\mathbb{R}^{d}\right)$ is even and convex function such that $c(0)=0$ and $A_{1}|x|^{q} \leq c(x) \leq A_{2}\left(|x|^{q}+1\right)$, for all $x \in \mathbb{R}^{d}$, with $1<q<\infty$.

$\left(H_{f_{0}}\right) f_{0}$ is a probability density on $\Omega \times \mathbb{R}^{d}$ such that

$$
\begin{aligned}
& \int_{\Omega \times \mathbb{R}^{d}}|v|^{q} f_{0}(x, v) \mathrm{d} x \mathrm{~d} v<\infty, \\
& \int_{\Omega \times \mathbb{R}^{d}} G\left(f_{0}(x, v)\right) \mathrm{d} x \mathrm{~d} v<\infty .
\end{aligned}
$$

$f_{0} \in L^{p}\left(\Omega \times \mathbb{R}^{d}\right)$ with $p=(q / q-1)$ and $N \leq f_{0} \leq M$ with $0<N$.

Remark 1. Typical examples satisfying assumption $\left(H_{G}\right)$ are the functions $G(s)=s \ln s, s>0$ and $G(s)=s^{r}, r>1$.

Proposition 1. Assume that $c$ satisfies $H_{c}$. Let $H:[0, \infty) \longrightarrow \mathbb{R}$ be a convex function such that $x \longmapsto x^{d} H\left(x^{-d}\right)$ is convex and decreasing.

Then, the functional

$$
\begin{aligned}
& E: P_{q}\left(\mathbb{R}^{d}\right) \longrightarrow \mathbb{R}, \\
& F \longmapsto \int_{\mathbb{R}^{d}} H(F) \mathrm{d} v,
\end{aligned}
$$

is displacement convex.

Proof. Let $F_{0}, F_{1} \in P_{q}\left(\mathbb{R}^{d}\right)$ and $T_{\#} F_{1}=F_{0}$ be a $c$-optimal map that pushes $F_{1}$ forward to $F_{0}$. We define $T_{t}=(1-$ $t) \mathrm{id}+t T$ and $F_{t}=T_{t \#} F_{1}$, where $t \in[0,1]$.

Then, we have

$$
E\left(F_{t}\right)=\int_{\mathbb{R}^{d}} H\left(F_{t}\right) \mathrm{d} v=\int_{F_{t}>0} \frac{H\left(F_{t}\right)}{F_{t}} F_{t} \mathrm{~d} v=\int_{\mathbb{R}^{d}} \frac{H\left(F_{t}^{\circ} T_{t}\right)}{F_{t} \circ T_{t}} F_{1} \mathrm{~d} v .
$$

From [1] and Proposition 3, we have that

$$
F_{1}=\operatorname{det}\left(\nabla T_{t}\right) F_{t} \circ T_{t} .
$$

Replacing (21) in (20), we obtain

$$
E\left(F_{t}\right)=\int_{\mathbb{R}^{d}} \frac{H\left(F_{1} / \operatorname{det} \nabla T_{t}\right)}{F_{1} / \operatorname{det} \nabla T_{t}} F_{1} \mathrm{~d} v .
$$

Recalling again [1] and Proposition 3, we get that $\nabla T_{t}$ is diagonalizable with positive eigenvalues. So, using the fact that the map $A \longmapsto(\operatorname{det} A)^{1 / d}$ is concave on the set of $d \times d$ diagonalizable matrices with positive eigenvalues, we get

$$
\left(\operatorname{det} \nabla T_{t}\right)^{1 / d} \geq(1-t)+t(\operatorname{det} \nabla T)^{1 / d} .
$$

Since $x \longmapsto x^{d} H\left(x^{-d}\right)$ is decreasing, then

$$
\begin{gathered}
\frac{H\left(F_{1} / \operatorname{det} \nabla T_{t}\right)}{F_{1} / \operatorname{det} \nabla T_{t}} \leq\left((1-t) F_{1}^{-1 / d}+t F_{1}^{-1 / d}(\operatorname{det} \nabla T)^{1 / d}\right)^{d} \\
H\left(\left((1-t) F_{1}^{-1 / d}+t F_{1}^{-1 / d}(\operatorname{det} \nabla T)^{\frac{1}{d}}\right)^{-d}\right) .
\end{gathered}
$$

From (23) and (24) and the fact that $x \longmapsto x^{d} H\left(x^{-d}\right)$ is convex, we obtain

$$
\begin{aligned}
E\left(F_{t}\right) & \leq(1-t) E\left(F_{1}\right)+t \int_{\mathbb{R}^{d}} \frac{H\left(F_{1} / \operatorname{det} \nabla T\right)}{F_{1} / \operatorname{det} \nabla T} F_{1} \mathrm{~d} v \\
& =(1-t) E\left(F_{1}\right)+t \int_{\mathbb{R}^{d}} H\left(F_{0}\right) \mathrm{d} v \\
& =(1-t) E\left(F_{1}\right)+t E\left(F_{0}\right) .
\end{aligned}
$$


Hence, we conclude that the functional $E$ is displacement convex.

Corollary 1. Since the functional $E$ is displacement convex, we have that

$$
E\left(F_{0}\right)-E\left(F_{1}\right) \geq\left.\frac{\mathrm{d} E\left(F_{t}\right)}{\mathrm{d} t}\right|_{t=0}=\int_{\mathbb{R}^{d}}\left\langle\nabla_{v}\left(H_{\prime}\left(F_{1}\right)\right), T(v)-v\right\rangle F_{1} \mathrm{~d} v .
$$

$F_{1}, F_{0} \in P_{q}\left(\mathbb{R}^{d}\right)$ and $T_{\#} F_{1}=F_{0}$ is the $c$-optimal map that pushes $F_{1}$ forward to $F_{0}$.

Definition 1. Let $f_{0} \in L^{1}\left(\Omega \times \mathbb{R}^{d}\right)$ be nonnegative. We say that a nonnegative function $f(t, x, v)$ on $[0, \infty) \times \Omega \times \mathbb{R}^{d}$ is a weak solution of (1) in the time's interval $[0, T]$ for some $T>0$, if for every test function $\psi \in C_{c}^{\infty}\left(\mathbb{R} \times \Omega \times \mathbb{R}^{d}\right)$ with time support $[-T, T]$, we have

$$
\int_{0}^{T} \int_{\Omega \times \mathbb{R}^{d}}\left[\partial_{t} \psi+v \cdot \nabla_{x} \psi-\nabla_{v} \psi \cdot \nabla c^{*}\left(\nabla_{v} G^{\prime}(f)\right)\right] f(t, x, v) \mathrm{d} t \mathrm{~d} x \mathrm{~d} v=-\int_{\Omega \times \mathbb{R}^{d}} f_{0}(x, v) \psi(0, x, v) \mathrm{d} x \mathrm{~d} v .
$$

2.1. The Flow and Descend Algorithm. Assume that the probability density $f_{0}(x, v)$ satisfies $\left(H_{f_{0}}\right)$ and fix $h>0$ a time step, then we define the following:

(1) $g_{1}^{h}(x, v):=f_{0}^{h}(x-h v, v)=f_{0}(x-h v, v)$

(2) $\rho_{1}^{h}(x):=\int_{\mathbb{R}^{d}} g_{1}^{h}(x, v) \mathrm{d} v$

(3) $G_{1}^{x h}(v):=g_{1}^{h}(x, v) / \rho_{1}^{h}(x)$ for $x \in \Omega$ fixed such that $\rho_{1}^{h}(x)>0$

(4) $f_{1}^{h}(x, v):=\rho_{1}^{h}(x) F_{1}^{x h}(v)$

For each $x$ fixed, $F_{1}^{x h}$ denotes the unique minimizer of the variational problem:

$$
\left(P_{1}^{x h}\right): \inf _{F \in P_{q}\left(\mathbb{R}^{d}\right)}\left\{I(F):=E(F)+h W_{c}^{h}\left(F, G_{1}^{x h}\right)\right\} .
$$

$P_{q}\left(\mathbb{R}^{d}\right)$ is the set of all probability density on $\mathbb{R}^{d}$ having a finite $q$-moment, and $W_{c}^{h}\left(F, G_{1}^{x h}\right)$ stands for the Kantorovich work defined as

$$
\begin{gathered}
W_{c}^{h}\left(F, G_{1}^{x h}\right)=\inf _{\gamma \in \Pi\left(F, G_{1}^{x h}\right)}\left\{\int_{\mathbb{R}^{d} \times \mathbb{R}^{d}} c\left(\frac{y-z}{h}\right) \mathrm{d} \gamma(y, z)\right\}, \\
E(F):=\frac{1}{\rho_{1}^{h}(x)} \int_{\mathbb{R}^{d}} G\left(\rho_{1}^{h}(x) F(v)\right) \mathrm{d} v .
\end{gathered}
$$

We obtain the terms $f_{k}^{h}$, for $k \geq 2$, by induction as follows:

(i) By fixing $v$, we define $g_{k}^{h}(x, v):=f_{k-1}^{h}(x-h v, v)$.

(ii) By fixing $x$, we define

$$
f_{k}^{h}(x, v):=\rho_{k}^{h}(x) F_{k}^{x h}(v)
$$

where

$$
\rho_{k}^{h}(x):=\int_{\mathbb{R}^{d}} g_{k}^{h}(x, v) \mathrm{d} v
$$

and $F_{k}^{x h}$ is the unique minimizer of the variational problem.

$$
\left(P_{k}^{x h}\right): \inf _{F \in P_{q}\left(\mathbb{R}^{d}\right)}\left\{I(F):=E(F)+h W_{c}^{h}\left(F, G_{k}^{x h}\right)\right\}
$$

with

$$
\begin{aligned}
G_{k}^{x}(v) & :=\frac{g_{k}^{h}(x, v)}{\rho_{k}^{h}(x)}, \\
E(F) & :=\frac{1}{\rho_{k}^{h}(x)} \int_{\mathbb{R}^{d}} G\left(\rho_{k}^{h}(x) F(v)\right) \mathrm{d} v .
\end{aligned}
$$

Existence of $F_{1}^{x h}$ and $F_{k}^{x h}$ will be proved farther in Sections 2 and 3, respectively.

2.2. $c$-Wasserstein Metric. In this section, we define a Wasserstein metric corresponding to a cost function $c$, and we study its topology.

Definition 2. Assume that $c: \mathbb{R}^{d} \longrightarrow[0, \infty)$ satisfies $H_{c}$. Let $\rho_{1}, \rho_{2} \in P\left(\mathbb{R}^{d}\right)$ two probability measures on $\mathbb{R}^{d}$. We define the $c$-Wasserstein metric between $\rho_{1}$ and $\rho_{2}$ by

$$
W_{c}\left(\rho_{1}, \rho_{2}\right):=\inf \left\{\lambda>0, \inf _{\gamma \in \Pi\left(\rho_{1}, \rho_{2}\right)} \int_{\mathbb{R}^{d} \times \mathbb{R}^{d}} c\left(\frac{x-y}{\lambda}\right) \mathrm{d} \gamma(x, y) \leq 1\right\} \text {. }
$$

Theorem 1. Assume that $c: \mathbb{R}^{d} \longrightarrow[0, \infty)$ satisfies $H_{c}$. Then, $W_{c}$ is a distance on the probability space $P\left(\mathbb{R}^{d}\right)$. Furthermore, if $\left(\rho_{n}\right)_{n}$ is a sequence in $P\left(\mathbb{R}^{d}\right)$ and $\rho \in P\left(\mathbb{R}^{d}\right)$, then $\left(\rho_{n}\right)_{n}$ converges to $\rho$ in the metric space $\left(P\left(\mathbb{R}^{d}\right), W_{c}\right)$ if and only if $\left(\rho_{n}\right)_{n}$ converges narrowly to $\rho$ in $P\left(\mathbb{R}^{d}\right)$.

Proof. Let $\rho_{1}, \rho_{2} \in P\left(\mathbb{R}^{d}\right)$ be two probability measures on $\mathbb{R}^{d}$ such that $W_{c}\left(\rho_{1}, \rho_{2}\right)=0$. Then, there exists a sequence $\left(\lambda_{n}\right)_{n}$ in $(0, \infty)$ which converges to 0 such that

$$
\inf _{\gamma \in \Pi\left(\rho_{1}, \rho_{2}\right)} \int_{\mathbb{R}^{d} \times \mathbb{R}^{d}} c\left(\frac{x-y}{\lambda_{n}}\right) \mathrm{d} \gamma \leq 1, \quad \text { for all } n \in \mathbb{N} .
$$

Denote by $\gamma_{n}$ the solution of Kantorovich problem: 


$$
\left(K_{n}\right): \inf _{\gamma \in \Pi\left(\rho_{1}, \rho_{2}\right)} \int_{\mathbb{R}^{d} \times \mathbb{R}^{d}} c\left(\frac{x-y}{\lambda_{n}}\right) \mathrm{d} \gamma .
$$

Then, we obtain

$$
\int_{\mathbb{R}^{d} \times \mathbb{R}^{d}} c\left(\frac{x-y}{\lambda_{n}}\right) \mathrm{d} \gamma_{n} \leq 1, \quad \text { for all } n \in \mathbb{N} .
$$

Since $\lambda_{n}$ converges to 0 , then $\left|x-y / \lambda_{n}\right|$ tends to $\infty$ and when $n$ goes to $\infty$, for all $x, y \in \mathbb{R}^{d}$ such that $x \neq y$. Then, using the fact that $c$ is coercive, we deduce that there exists $N_{0} \in \mathbb{N}$ such that

$$
c\left(\frac{x-y}{\lambda_{n}}\right) \geq\left|\frac{x-y}{\lambda_{n}}\right|, \quad \text { for all } n \geq N_{0} .
$$

This with (37) implies that

$$
\inf _{\gamma \in \Pi\left(\rho_{1}, \rho_{2}\right)} \int_{\mathbb{R}^{d} \times \mathbb{R}^{d}}|x-y| \mathrm{d} \gamma \leq \lambda_{n}, \quad \text { for all } n \geq N_{0} .
$$

Thus, we conclude that

$$
\inf _{\gamma \in \Pi\left(\rho_{1}, \rho_{2}\right)} \int_{\mathbb{R}^{d} \times \mathbb{R}^{d}}|x-y| \mathrm{d} \gamma=0 .
$$

Let $\gamma_{0}$ be the solution of the Kantorovich problem:

$$
\left(K_{0}\right): \inf _{\gamma \in \Pi\left(\rho_{1}, \rho_{2}\right)} \int_{\mathbb{R}^{d} \times \mathbb{R}^{d}}|x-y| \mathrm{d} \gamma .
$$

Then, using (40), we obtain

$$
\int_{\mathbb{R}^{d} \times \mathbb{R}^{d}}|x-y| \mathrm{d} \gamma_{0}=0 .
$$

We deduce that $x=y \gamma_{0}$ a.e. So for all $\phi \in C_{b}\left(\mathbb{R}^{d}\right)$, we have

$$
\begin{aligned}
\int_{\mathbb{R}^{d}} \phi(x) \rho_{1} \mathrm{~d} x & =\int_{\mathbb{R}^{d} \times \mathbb{R}^{d}} \phi(x) \mathrm{d} \gamma_{0}(x, y) \\
& =\int_{\mathbb{R}^{d} \times \mathbb{R}^{d}} \phi(y) \mathrm{d} \gamma_{0}(x, y)=\int_{\mathbb{R}^{d}} \phi(y) \rho_{2} \mathrm{~d} y .
\end{aligned}
$$

Consequently, $\rho_{1}=\rho_{2}$.

Let us fix two probability measures $\rho_{1}$ and $\rho_{2}$ on $\mathbb{R}^{d}$. Since $c$ is even, then

$$
\inf _{\gamma \in \Pi\left(\rho_{1}, \rho_{2}\right)} \int_{\mathbb{R}^{d} \times \mathbb{R}^{d}} c\left(\frac{x-y}{\lambda}\right) \mathrm{d} \gamma=\inf _{\bar{\gamma} \in \Pi\left(\rho_{2}, \rho_{1}\right)} \int_{\mathbb{R}^{d} \times \mathbb{R}^{d}} c\left(\frac{x-y}{\lambda}\right) \mathrm{d} \bar{\gamma},
$$

for all $\lambda>0$. We deduce from (44) that

$$
\begin{aligned}
W_{c}\left(\rho_{1}, \rho_{2}\right) & =\inf \left\{\lambda>0, \inf _{\gamma \in \Pi\left(\rho_{1}, \rho_{2}\right)} \int_{\mathbb{R}^{d} \times \mathbb{R}^{d}} c\left(\frac{x-y}{\lambda}\right) \mathrm{d} \gamma(x, y) \leq 1\right\} \\
& =\inf \left\{\lambda>0, \inf _{\bar{\gamma} \in \Pi\left(\rho_{2}, \rho_{1}\right)} \int_{\mathbb{R}^{d} \times \mathbb{R}^{d}} c\left(\frac{x-y}{\lambda}\right) \mathrm{d} \bar{\gamma}(x, y) \leq 1\right\} \\
& =W_{c}\left(\rho_{2}, \rho_{1}\right) .
\end{aligned}
$$

Let $\rho_{1}, \rho_{2}, \rho_{3}$ be three probability measures on $\mathbb{R}^{d}$. Define $\lambda_{1}=W_{c}\left(\rho_{1}, \rho_{2}\right)$ and $\lambda_{2}=W_{c}\left(\rho_{2}, \rho_{3}\right)$. Denote by $\gamma_{1} \in \Pi\left(\rho_{1}, \rho_{2}\right)$ the solution of Kantorovich problem

$$
\left(K_{1}\right): \inf _{\gamma \in \Pi\left(\rho_{1}, \rho_{2}\right)} \int_{\mathbb{R}^{d} \times \mathbb{R}^{d}} c\left(\frac{x-y}{\lambda_{1}}\right) \mathrm{d} \gamma,
$$

and denote by $\gamma_{2} \in \Pi\left(\rho_{2}, \rho_{3}\right)$ the solution of the Kantorovich problem:

$$
\left(K_{2}\right): \inf _{\gamma \in \Pi\left(\rho_{2}, \rho_{3}\right)} \int_{\mathbb{R}^{d} \times \mathbb{R}^{d}} c\left(\frac{x-y}{\lambda_{2}}\right) \mathrm{d} \gamma .
$$

Using the Gluing lemma [8], there exists a probability measure $\sigma$ on $\mathbb{R}^{d} \times \mathbb{R}^{d} \times \mathbb{R}^{d}$ such that

$$
\begin{aligned}
& \sigma\left(A \times B \times \mathbb{R}^{d}\right)=\gamma_{1}(A \times B), \\
& \sigma\left(\mathbb{R}^{d} \times A \times B\right)=\gamma_{2}(A \times B),
\end{aligned}
$$

for some Borel subsets $A$ and $B$ of $\mathbb{R}^{d}$. Let $\gamma_{3}$ be a probability measure on $\mathbb{R}^{d} \times \mathbb{R}^{d}$ defined by $\gamma_{3}(A \times B)=\sigma\left(A \times \mathbb{R}^{d} \times B\right)$. Then, $\gamma_{3} \in \Pi\left(\rho_{1}, \rho_{3}\right)$, and we use the convexity of $c$ to get that

$$
\begin{aligned}
& \int_{\mathbb{R}^{d} \times \mathbb{R}^{d}} c\left(\frac{x-z}{\lambda_{1}+\lambda_{2}}\right) \mathrm{d} \gamma_{3}=\int_{\mathbb{R}^{d} \times \mathbb{R}^{d} \times \mathbb{R}^{d}} c\left(\frac{x-z}{\lambda_{1}+\lambda_{2}}\right) \mathrm{d} \sigma \\
& \leq \frac{\lambda_{1}}{\lambda_{1}+\lambda_{2}} \int_{\mathbb{R}^{d} \times \mathbb{R}^{d}} c\left(\frac{x-y}{\lambda_{1}}\right) \mathrm{d} \gamma_{1}+\frac{\lambda_{2}}{\lambda_{1}+\lambda_{2}} \int_{\mathbb{R}^{d} \times \mathbb{R}^{d}} c\left(\frac{y-z}{\lambda_{2}}\right) \mathrm{d} \gamma_{2} \\
& \leq \frac{\lambda_{1}}{\lambda_{1}+\lambda_{2}}+\frac{\lambda_{2}}{\lambda_{1}+\lambda_{2}}=1 .
\end{aligned}
$$

So, $\quad \inf _{\gamma \in \Pi\left(\rho_{1}, \rho_{3}\right)} \int_{\mathbb{R}^{d} \times \mathbb{R}^{d}} c\left(x-z / \lambda_{1}+\lambda_{2}\right) \mathrm{d} \gamma \leq 1$, and we conclude that

$$
W_{c}\left(\rho_{1}, \rho_{2}\right) \leq \lambda_{1}+\lambda_{2}=W_{c}\left(\rho_{1}, \rho_{2}\right)+W_{c}\left(\rho_{2}, \rho_{3}\right) .
$$

Hence, $W_{c}$ is a distance on $P\left(\mathbb{R}^{d}\right)$.

Let us now study the topology of $W_{c}$.

Let $\left(\rho_{n}\right)_{n}$ be a sequence on $P\left(\mathbb{R}^{d}\right)$ and $\rho \in P\left(\mathbb{R}^{d}\right)$ such that $W_{c}\left(\rho_{n}, \rho\right)$ converges to 0 when $n$ tends to $\infty$. Define $\lambda_{n}=W_{c}\left(\rho_{n}, \rho\right)$, since $\left(\lambda_{n}\right)_{n}$ converges to 0 , then we use the fact that $c$ is coercive to have

$$
\inf _{\gamma \in \Pi\left(\rho_{n}, \rho\right)} \int_{\mathbb{R}^{d} \times \mathbb{R}^{d}}|x-y| \mathrm{d} \gamma \leq \lambda_{n},
$$

when $n \longrightarrow \infty$. We deduce that

$$
\limsup _{n \rightarrow \infty} \inf _{\gamma \in \Pi\left(\rho_{n}, \rho\right)} \int_{\mathbb{R}^{d} \times \mathbb{R}^{d}}|x-y| \mathrm{d} \gamma=0 \text {. }
$$

Note that the 1-Wasserstein metric between $\rho_{n}$ and $\rho$ is

$$
W_{1}\left(\rho_{n}, \rho\right)=\inf _{\gamma \in \Pi\left(\rho_{n}, \rho\right)} \int_{\mathbb{R}^{d} \times \mathbb{R}^{d}}|x-y| \mathrm{d} \gamma .
$$

We deduce that $W_{1}\left(\rho_{n}, \rho\right)$ converges to 0 when $n$ tends to $\infty$. Since the 1 -Wasserstein metric $W_{1}$ induces the narrow topology of $P\left(\mathbb{R}^{d}\right)$, we conclude that the sequence $\left(\rho_{n}\right)_{n}$ converges narrowly to $\rho$ in $P\left(\mathbb{R}^{d}\right)$. 
Assume now that the sequence $\left(\rho_{n}\right)_{n}$ converges narrowly to $\rho$ in $P\left(\mathbb{R}^{d}\right)$. Fix $\lambda>0$ and denote by $\gamma_{n}^{\lambda}$ the solution of Kantorovich problem:

$$
(K): \inf _{\gamma \in \Pi\left(\rho_{n}, \rho\right)} \int_{\mathbb{R}^{d} \times \mathbb{R}^{d}} c\left(\frac{x-y}{\lambda}\right) \mathrm{d} \gamma .
$$

Since $\rho_{n}$ converges narrowly to $\rho$, then $\gamma_{n}^{\lambda}$ converges narrowly to some $\gamma^{\lambda} \in \Pi(\rho, \rho)$ and

$$
\lim _{n \longrightarrow \infty} \int_{\mathbb{R}^{d} \times \mathbb{R}^{d}} c\left(\frac{x-y}{\lambda}\right) \mathrm{d} \gamma_{n}^{\lambda}=\int_{\mathbb{R}^{d} \times \mathbb{R}^{d}} c\left(\frac{x-y}{\lambda}\right) \mathrm{d} \gamma^{\lambda}=0 .
$$

So,

$$
\lim _{n \longrightarrow \infty} \inf _{\gamma \in \Pi\left(\rho_{n}, \rho\right)} \int_{\mathbb{R}^{d} \times \mathbb{R}^{d}} c\left(\frac{x-y}{\lambda}\right) \mathrm{d} \gamma=0,
$$

for all $\lambda>0$. Hence, for all $\lambda>0$, there exists $N_{\lambda}>0$ such that

$$
\inf _{\gamma \in \Pi\left(\rho_{n}, \rho\right)} \int_{\mathbb{R}^{d} \times \mathbb{R}^{d}} c\left(\frac{x-y}{\lambda}\right) \mathrm{d} \gamma \leq 1, \quad \text { for all } n \geq N_{\lambda} .
$$

So, $W_{c}\left(\rho_{n}, \rho\right) \leq \lambda$ for all $n \geq N_{\lambda}$. Then,

$$
\lim _{n \longrightarrow \infty} W_{c}\left(\rho_{n}, \rho\right)=0 \text {. }
$$

Consequently, $\left(\rho_{n}\right)_{n}$ converges narrowly to $\rho$ in the metric space $\left(P(\Omega), W_{c}\right)$.

We establish now the existence of solution for the variational problem $(P)$ defined by

$$
(P): \inf _{\rho \in P_{q}\left(\mathbb{R}^{d}\right)}\left\{\int_{\mathbb{R}^{d}} G(\rho) \mathrm{d} x+h W_{c}^{h}\left(\rho_{0}, \rho\right)\right\},
$$

in the metric space $\left(P\left(\mathbb{R}^{d}\right), W_{c}\right)$, where

$$
W_{c}^{h}\left(\rho_{0}, \rho\right)=\inf _{\gamma \in \Pi\left(\rho_{0}, \rho\right)} \int_{\mathbb{R}^{d} \times \mathbb{R}^{d}} c\left(\frac{x-y}{h}\right) \mathrm{d} \gamma .
$$

$P_{q}\left(\mathbb{R}^{d}\right)$ is the set of all probability measures on $\mathbb{R}^{d}$ having $q$-finite moment, that is,

$$
P_{q}\left(\mathbb{R}^{d}\right)=\left\{\rho \in P\left(\mathbb{R}^{d}\right), \int_{\mathbb{R}^{d}}|x|^{q} \rho \mathrm{d} x<\infty\right\},
$$

and $h>0$ being a time step.

Lemma 1. Assume that $\rho_{0}, G$, and c satisfy, respectively, $H_{f_{0}}$, $H_{G}$, and $H_{c}$. Then, the following is obtained:

(i) The map $\rho \longrightarrow W_{c}^{h}\left(\rho_{0}, \rho\right)$ is lower semicontinuous in $\left(P\left(\mathbb{R}^{d}\right), W_{c}\right)$.

(ii) The functional $\rho \longrightarrow \int_{\mathbb{R}^{d}} G(\rho) d x$ is lower semicontinuous in $L^{1}\left(\mathbb{R}^{d}\right)$.

(iii) The set $\left\{\rho \in P_{M}\left(\mathbb{R}^{d}\right), \int_{\mathbb{R}^{d}} G(\rho) d x \leq \int_{\mathbb{R}^{d}} G\left(\rho_{0}\right) d x\right\}$ is a closed subset of $L^{1}\left(\mathbb{R}^{d}\right)$,

$$
\text { where } P_{M}\left(\mathbb{R}^{d}\right)=\left\{\rho \in P\left(\mathbb{R}^{d}\right), \rho \leq M\right\} \text {. }
$$

Proof

(i) Let $\rho \in P\left(\mathbb{R}^{d}\right)$. Let $\left(\rho_{n}\right)_{n}$ be a sequence in $P\left(\mathbb{R}^{d}\right)$ such that $\left(\rho_{n}\right)_{n}$ converges to $\rho$ in metric space
$\left(P\left(\mathbb{R}^{d}\right), W_{c}\right)$. Denote by $\gamma_{n}^{h}$ the solution of the Kantorovich problem:

$$
\left(K_{n}^{h}\right): \inf _{\gamma \in \Pi\left(\rho_{n}, \rho_{0}\right)} \int_{\mathbb{R}^{d} \times \mathbb{R}^{d}} c\left(\frac{x-y}{h}\right) \mathrm{d} \gamma .
$$

We have

$$
\inf _{\gamma \in \Pi\left(\rho_{n}, \rho_{0}\right)} \int_{\mathbb{R}^{d} \times \mathbb{R}^{d}} c\left(\frac{x-y}{h}\right) \mathrm{d} \gamma=\int_{\mathbb{R}^{d} \times \mathbb{R}^{d}} c\left(\frac{x-y}{h}\right) \mathrm{d} \gamma_{n}^{h}
$$

Since $\left(\rho_{n}\right)_{n}$ converges to $\rho$ narrowly, then $\left(\gamma_{n}^{h}\right)_{n}$ converges to $\gamma^{h} \in \Pi\left(\rho_{0}, \rho\right)$ narrowly and

$$
\liminf _{n \longrightarrow \infty} \int_{\mathbb{R}^{d} \times \mathbb{R}^{d}} c\left(\frac{x-y}{h}\right) \mathrm{d} \gamma_{n}^{h} \geq \int_{\mathbb{R}^{d} \times \mathbb{R}^{d}} c\left(\frac{x-y}{h}\right) \mathrm{d} \gamma^{\widehat{h}} \geq W_{c}^{h}\left(\rho_{0}, \rho\right)
$$

This implies that

$$
\liminf _{n \rightarrow \infty} W_{c}^{h}\left(\rho_{0}, \rho_{n}\right) \geq W_{c}^{h}\left(\rho_{0}, \rho\right)
$$

Thus, we obtain the proof of (i).

(ii) Since $G$ is convex and $G \in C^{1}((0, \infty))$,

$$
\int_{\mathbb{R}^{d}} G\left(\rho_{n}\right) \mathrm{d} x \geq \int_{\mathbb{R}^{d}} G(\rho) \mathrm{d} x+\int_{\mathbb{R}^{d}} G^{\prime}(\rho)\left(\rho_{n}-\rho\right) \mathrm{d} x .
$$

Hence, if $\left(\rho_{n}\right)_{n}$ converges to $\rho$ weakly in $L^{1}\left(\mathbb{R}^{d}\right)$ with $\rho \in L^{\infty}\left(\mathbb{R}^{d}\right)$, we have

$$
\liminf _{n \rightarrow \infty} \int_{\mathbb{R}^{d}} G^{\prime}(\rho)\left(\rho_{n}-\rho\right) \mathrm{d} x=0,
$$

and then

$$
\liminf _{n \longrightarrow \infty} \int_{\mathbb{R}^{d}} G\left(\rho_{n}\right) \mathrm{d} x \geq \int_{\mathbb{R}^{d}} G(\rho) \mathrm{d} x,
$$

which complete the proof of (ii). The proof of (iii) is a consequence of (ii).

2.3. Existence Results for the Discrete Problem $\left(P_{1}^{x h}\right)$. First of all, we introduce here for unbounded domains analogous of the maximum principle stated for bounded domains in [1]. This maximum principle plays a central role in the searching of solution for the discrete problem $\left(P_{1}^{x h}\right)$. It is also used to further establish the convergence of our algorithm towards a weak solution of the kinetic equation (1).

Proposition 2 (maximum principle). Assume that the initial datum $f_{0}$ satisfies $N \leq f_{0} \leq M$ with $0<N$, c satisfies $\left(H_{c}\right)$, and $G$ satisfies $\left(H_{G}\right)$.

Then, any solution $F_{1}^{x h}$ of the variational problem 


$$
\left(P_{1}^{x h}\right): \inf _{F \in P_{q}\left(\mathbb{R}^{d}\right)}\left\{E(F)+h W_{c}^{h}\left(F, G_{1}^{x h}\right)\right\},
$$

satisfies $a(x) \leq F_{1}^{x h} \leq b(x)$, with $a(x)=\left(N / \rho_{1}^{h}(x)\right)$ and $b(x)=\left(M / \rho_{1}^{h}(x)\right)$.

Proof. We define $E_{x}:=\left\{y \in \mathbb{R}^{d}, F_{1}^{x h}>b(x)\right\}$. Assume by contradiction that $E_{x}$ has a positive Lebesgue measure. Then, $\gamma_{x}\left(E_{x}^{c} \times E_{x}\right)>0$, where $\gamma_{x}$ is the minimizer of

$$
(K): \inf _{\gamma \in \Pi\left(G_{1}^{x h}, F_{1}^{x h}\right)}\left\{\int_{\mathbb{R}^{d} \times \mathbb{R}^{d}} c\left(\frac{y-z}{h}\right) \mathrm{d} \gamma(y, z)\right\} .
$$

Otherwise,

$$
\begin{gathered}
b(x)\left|E_{x}\right|<\int_{E_{x}} F_{1}^{x h} \mathrm{~d} v=\gamma_{x}\left(\mathbb{R}^{d} \times E_{x}\right)=\gamma_{x}\left(E_{x} \times E_{x}\right) \\
\leq \gamma_{x}\left(E_{x} \times \mathbb{R}^{d}\right)=\int_{E_{x}} G_{1}^{x h} \mathrm{~d} y \leq\left|E_{x}\right| b(x) .
\end{gathered}
$$

This yields a contradiction.

Define $v_{x}=1_{E_{x}^{c} \times E_{x}} \gamma_{x}$, i.e., $v_{x}(F)=\gamma_{x}\left(F \cap E_{x}^{c} \times E_{x}\right)$, for all $F \subset \mathbb{R}^{d}$, and denote by $\nu_{0, x}$ and $\nu_{1, x}$ the marginals of $\nu_{x}$. We have $v_{x} \ll \gamma_{x}$ with $\gamma_{x} \in \Pi\left(G_{1}^{x h}, F_{1}^{x h}\right)$ and then $\nu_{0, x} \ll G_{1}^{x h} \mathrm{~d} y$ and $\nu_{1, x} \ll F_{1}^{x h} \mathrm{~d} z$.

Let $v_{0, x}$ and $v_{1, x}$ denote, respectively, the density functions of $v_{0, x}$ and $v_{1, x}$. Because

$$
\begin{aligned}
& \int_{E_{x}} v_{0, x} d y=v_{0, x}\left(E_{x}\right)=\gamma_{x}\left(\left(E_{x} \times \mathbb{R}^{d}\right) \cap E_{x}^{c} \times E_{x}\right)=0, \\
& \int_{E_{x}^{c}} v_{1, x} \mathrm{~d} y=v_{1, x}\left(E_{x}^{c}\right)=\gamma_{x}\left(\left(\mathbb{R}^{d} \times E_{x}^{c}\right) \cap E_{x}^{c} \times E_{x}\right)=0,
\end{aligned}
$$

we have $v_{0, x}=0$ on $E_{x}$ and $v_{1, x}=0$ on $E_{x}^{c}$.

For $\varepsilon>0$ is small enough, define $F_{1}^{x h, \varepsilon}=F_{1}^{x h}+\varepsilon\left(v_{0, x}-\right.$ $\left.v_{1, x}\right)$ and $\gamma_{x}^{\epsilon}$ by

$$
\begin{gathered}
\int_{\mathbb{R}^{d} \times \mathbb{R}^{d}} H(x, y) \mathrm{d} \gamma_{x}^{\varepsilon}=\int_{\mathbb{R}^{d} \times \mathbb{R}^{d}} H(x, y) \mathrm{d} \gamma_{x} \\
+\varepsilon \int_{E_{x}^{c} \times E_{x}}[H(x, x)-H(x, y)] \mathrm{d} \gamma_{x},
\end{gathered}
$$

for all $H \in C_{0}\left(\mathbb{R}^{d} \times \mathbb{R}^{d}\right)$.

$F_{1}^{x h, \varepsilon} \in P_{q}\left(\mathbb{R}^{d}\right), \gamma_{x}^{\varepsilon} \in \Pi\left(G_{1}^{x h}, F_{1}^{x h, \varepsilon}\right)$, and we get

$$
\begin{aligned}
& I\left(F_{1}^{x h, \varepsilon}\right)-I\left(F_{1}^{x h}\right)=h\left[W_{c}^{h}\left(F_{1}^{x h, \varepsilon}, G_{1}^{x h}\right)\right. \\
& \left.\quad-W_{c}^{h}\left(F_{1}^{x h}, G_{1}^{x h}\right)\right]+\frac{1}{\rho_{1}^{h}(x)} \int_{\mathbb{R}^{d}}\left[G\left(\rho_{1}^{h}(x) F_{1}^{x h, \varepsilon}\right)-G\left(\rho_{1}^{h}(x) F_{1}^{x h}\right)\right] .
\end{aligned}
$$

Taking into account some ideas from [1], we shall prove that $I\left(F_{1}^{x h, \epsilon}\right)-I\left(F_{1}^{x h}\right)<0$, which leads to a contradiction.

Indeed,

$$
\begin{aligned}
& W_{c}^{h}\left(F_{1}^{x h, \varepsilon}, G_{1}^{x h}\right)-W_{c}^{h}\left(F_{1}^{x h}, G_{1}^{x h}\right) \\
& =\inf _{\gamma \in \Pi\left(F_{1}^{x h,}, F_{1}^{x h}\right)} \int_{\mathbb{R}^{d} \times \mathbb{R}^{d}} c\left(\frac{y-z}{h}\right) \mathrm{d} \gamma-\int_{\mathbb{R}^{d} \times \mathbb{R}^{d}} c\left(\frac{y-z}{h}\right) \mathrm{d} \gamma_{x} \\
& \leq \int_{\mathbb{R}^{d} \times \mathbb{R}^{d}} c\left(\frac{y-z}{h}\right) \mathrm{d} \gamma_{x}^{\varepsilon}-\int_{\mathbb{R}^{d} \times \mathbb{R}^{d}} c\left(\frac{y-z}{h}\right) \mathrm{d} \gamma_{x} \\
& =-\varepsilon \int_{E_{x}^{c} \times E_{x}} c\left(\frac{y-z}{h}\right) \mathrm{d} \gamma_{x} .
\end{aligned}
$$

Since $(y, z) \longmapsto c(y-z / h)$ is nonnegative on $E_{x}^{c} \times E_{x}$ and $\gamma_{x}\left(E_{x}^{c} \times E_{x}\right)>0$, we have

$$
W_{c}^{h}\left(F_{1}^{x h, \varepsilon}, G_{1}^{x h}\right)-W_{c}^{h}\left(F_{1}^{x h}, G_{1}^{x h}\right)=-\varepsilon \int_{E_{x}^{c} \times E_{x}} c\left(\frac{y-z}{h}\right) \mathrm{d} \gamma_{x}<0 .
$$

Also, we have

$$
\begin{aligned}
& \frac{1}{\rho_{1}^{h}(x)} \int_{\mathbb{R}^{d}}\left[G\left(\rho_{1}^{h}(x) F_{1}^{x h, \varepsilon}\right)-G\left(\rho_{1}^{h}(x) F_{1}^{x h}\right)\right] \mathrm{d} v \\
& =\frac{1}{\rho_{1}^{h}(x)} \int_{\mathbb{R}^{d}}\left[G\left(\rho_{1}^{h}(x) F_{1}^{x h}+\varepsilon \rho_{1}^{h}(x)\left(v_{0, x}-v_{1, x}\right)\right)-G\left(\rho_{1}^{h}(x) F_{1}^{x h}\right)\right] \mathrm{d} v \\
& =\frac{1}{\rho_{1}^{h}(x)} \int_{E_{x}}\left[G\left(\left(\rho_{1}^{h}(x) F_{1}^{x h}-\varepsilon \rho_{1}^{h} v_{1, x}\right)-G\left(\rho_{1}^{h}(x) F_{1}^{x h}\right)\right)\right] \mathrm{d} v \\
& +\frac{1}{\rho_{1}^{h}(x)} \int_{E_{x}^{c}}\left[G\left(\rho_{1}^{h}(x) F_{1}^{x h}+\varepsilon \rho_{1}^{h}(x) v_{0, x}\right)-G\left(\rho_{1}^{h}(x) F_{1}^{x h}\right)\right] \mathrm{d} v .
\end{aligned}
$$

$G$ is being convex and of class $C^{1}$, then

$$
G\left(\rho_{1}^{h} F_{1}^{x h}\right)-G\left(\rho_{1}^{h} F_{1}^{x h}-\varepsilon \rho_{1}^{h} v_{1, x}\right) \geq G^{\prime}\left(\rho_{1}^{h} F_{1}^{x h}-\varepsilon \rho_{1}^{h} v_{1, x}\right) \varepsilon \rho_{1}^{h} v_{1, x},
$$

on $E_{x}$ and

$$
\begin{aligned}
& G\left(\rho_{1}^{h}(x) F_{1}^{x h}+\varepsilon \rho_{1}^{h}(x) v_{0, x}\right)-G\left(\rho_{1}^{h}(x) F_{1}^{x h}\right) \\
& \quad \leq \varepsilon \rho_{1}^{h} v_{0, x} G^{\prime}\left(\rho_{1}^{h} F_{1}^{x h}+\varepsilon \rho_{1}^{h} v_{0, x}\right)
\end{aligned}
$$

on $E_{x}^{c}$.

Hence, we have

$$
\begin{aligned}
& \frac{1}{\rho_{1}^{h}(x)} \int_{\mathbb{R}^{d}}\left[G\left(\rho_{1}^{h}(x) F_{1}^{x h, \varepsilon}\right)-G\left(\rho_{1}^{h}(x) F_{1}^{x h}\right)\right] \mathrm{d} v \\
& \leq \varepsilon\left[\int _ { E _ { x } ^ { c } } \left[G^{\prime}\left(\rho_{1}^{h} F_{1}^{x h}+\varepsilon \rho_{1}^{h} v_{0, x}\right) v_{0, x} \mathrm{~d} v\right.\right. \\
& \quad-\int_{E_{x}}\left[G^{\prime}\left(\rho^{h} F_{1}^{x h}-\varepsilon \rho_{1}^{h} v_{1, x}\right) v_{1, x} \mathrm{~d} v\right] \\
& =\varepsilon \int_{E_{x}^{c} \times E_{x}}\left[G^{\prime}\left(\rho_{1}^{h} F_{1}^{x h}+\varepsilon \rho_{1}^{h} v_{0, x}\right)-G^{\prime}\left(\rho_{1}^{h} F_{1}^{x h}-\varepsilon \rho_{1}^{h} v_{1, x}\right)\right] \mathrm{d} \gamma_{x} .
\end{aligned}
$$

Since $G^{\prime}$ is continuous, then 


$$
\lim _{\varepsilon \longrightarrow 0}\left[G^{\prime}\left(\rho_{1}^{h} F_{1}^{x h}+\varepsilon \rho_{1}^{h} v_{0, x}\right)-G^{\prime}\left(\rho_{1}^{h} F_{1}^{x h}-\varepsilon \rho_{1}^{h} v_{1, x}\right)\right]=0 .
$$
then

Consequently, there exists $\delta>0$ such that, for $0<\varepsilon<\delta$,

$$
\begin{aligned}
0 & \leq G^{\prime}\left(\rho_{1}^{h} F_{1}^{x h}+\epsilon \rho_{1}^{h} v_{0, x}\right)-G^{\prime}\left(\rho_{1}^{h} F_{1}^{x h}-\epsilon \rho_{1}^{h} v_{1, x}\right) \\
& \leq \frac{1}{2 \gamma_{x}\left(E_{x}^{c} \times E_{x}\right)} \int_{E_{x}^{c} \times E_{x}} c\left(\frac{y-z}{h}\right) d \gamma_{x}
\end{aligned}
$$

So, we fix $\varepsilon>0$ small, such that $0<\varepsilon<\delta$, and we use (81) and (83) to obtain

$$
\begin{aligned}
& \frac{1}{\rho_{1}^{h}(x)} \int_{\mathbb{R}^{d}}\left[G\left(\rho_{1}^{h}(x) F_{1}^{x h, \varepsilon}\right)-G\left(\rho_{1}^{h}(x) F_{1}^{x h}\right)\right] \mathrm{d} v \\
& \leq \frac{\varepsilon}{2} \int_{E_{x}^{c} \times E_{x}} c\left(\frac{y-z}{h}\right) \mathrm{d} \gamma_{x} .
\end{aligned}
$$

Now, fixing $\epsilon<\delta$ and combining (77) and (84) yield

$$
\begin{aligned}
& I\left(F_{1}^{x h, \varepsilon}\right)-I\left(F^{x h}\right) \\
& <-\varepsilon \int_{E_{x}^{c} \times E_{x}} c\left(\frac{y-z}{h}\right) \mathrm{d} \gamma_{x}+\frac{\varepsilon}{2} \int_{E_{x}^{c} \times E_{x}} c\left(\frac{y-z}{h}\right) \mathrm{d} \gamma_{x} \\
& =-\frac{\varepsilon}{2} \int_{E_{x}^{c} \times E_{x}} c\left(\frac{y-z}{h}\right) \mathrm{d} \gamma_{x}<0 .
\end{aligned}
$$

It is a contradiction since $F_{1}^{x h}$ is a minimizer of $I$ on $P_{q}\left(\mathbb{R}^{d}\right)$. Consequently, $E_{x}$ is negligible and then $F_{1}^{x h} \leq b(x)$.

The proof of $F_{1}^{x h} \geq a(x)$ is analogous to that of $F_{1}^{x} \leq b(x)$. Thus, we conclude that $a(x) \leq F_{1}^{x h} \leq b(x)$.

Lemma 2. Let $f_{0} \in P_{q}\left(\Omega \times \mathbb{R}^{d}\right)$ be a probability density on $\Omega \times \mathbb{R}^{d}$ such that $0<N \leq f_{0} \leq M, \int_{\Omega \times \mathbb{R}^{d}} G\left(f_{0}\right) d x d v<\infty$ and $\int_{\Omega \times \mathbb{R}^{d}}|v|^{q} f_{0}(x, v) d x d v<\infty$.

The variational problem $\left(P_{1}^{x h}\right)$ defined in (32) admits a unique minimizer $F_{1}^{x h}$ in $P_{q}\left(\mathbb{R}^{d}\right)$ and $\left(N / \rho_{1}^{h}(x)\right) \leq F_{1}^{x h} \leq\left(M / \rho_{1}^{h}(x)\right)$.

Proof. Since $0<N \leq f_{0} \leq M$ and $G_{1}^{x h}(v)=f_{0}(x, v) / \rho_{1}^{h}(x)$, then $\left(N / \rho_{1}^{h}(x)\right) \leq G_{1}^{x h} \leq\left(M / \rho_{1}^{h}(x)\right)$. Then, we use Proposition 2, and we obtain that any minimizer $F_{1}^{x h}$ of the variational problem $P_{1}^{x h}$ satisfies $a(x) \leq F_{1}^{x h} \leq b(x)$, with $a(x)=N / \rho_{1}^{h}(x)$ and $b(x)=M / \rho_{1}^{h}(x)$.

By using Proposition 2 and the fact that $G$ is convex, we obtain that

$$
E(F) \geq 1 / \rho_{1}^{h}(x) \int_{\mathbb{R}^{d}} G\left(\rho_{1}^{h}(x) G_{1}^{x h}(v)\right) \mathrm{d} v+G^{\prime}(N)-G^{\prime}(M),
$$

for all probability density $F \in P_{q}\left(\mathbb{R}^{d}\right)$ such that $N \leq F \leq M$. We use now Lemma 1 to get that the functional
$F \longmapsto 1 / \rho^{h}(x) \int_{\mathbb{R}^{d}} G\left(\rho^{h}(x) F(v)\right) \mathrm{d} v$ is lower semicontinuous on the Wasserstein space $\left(P_{q}\left(\mathbb{R}^{d}\right), W_{c}\right)$. We conclude then that the problem $\left(P_{1}^{x h}\right)$ admits a solution $F_{1}^{x h}$. The strict convexity of $G$ and $c$ implies the strict convexity of the map $F \longmapsto W_{c}^{h}\left(G_{1}^{x h}, F\right)$ and that of the maps $F \longmapsto 1 / \rho^{h}(x) \int_{\mathbb{R}^{d}} G\left(\rho^{h}(x) F(v)\right) \mathrm{d} v$ and accordingly the uniqueness of the minimizer $F_{1}^{x h}$ of $\left(P_{1}^{x h}\right)$.

\section{Euler-Lagrange Equation for the Problem $\left(P_{k}^{x h}\right)$}

In this section, we prove that the sequence $\left(f_{k}^{h}\right)_{k}$ is a time discretization of the kinetic equation (1). In order to achieve it, we need the following lemma.

Lemma 3 (explicit expression for optimal maps). Assume that $G$ satisfies $H_{G}$ and $c$ satisfies $H_{c}$. Then, the Monge problem

$$
(M): \inf _{T_{\#} F_{k}^{x h}=G_{k}^{x h}} \int_{\mathbb{R}^{d}} c\left(\frac{v-T(v)}{h}\right) F_{k}^{x h} \mathrm{~d} v,
$$

admits a unique solution $T_{k}$ such that

$$
T_{k}(v)=v+h \nabla c^{*}\left(\nabla_{v} G^{\prime}\left(\rho_{k}^{h} F_{k}^{x h}\right)\right),
$$

where $F_{k}^{x}$ is the unique minimizer of the variational problem.

$$
\left(P_{k}^{x h}\right): \inf _{F \in P_{q}\left(\mathbb{R}^{d}\right)}\left\{I(F):=\frac{1}{\rho_{k}^{h}} \int_{\mathbb{R}^{d}} G\left(\rho_{k}^{h} F\right)+h W_{c}^{h}\left(G_{k}^{x h}, F\right)\right\} .
$$

Proof. Let $\psi \in C_{c}^{\infty}\left(\mathbb{R}^{d}, \mathbb{R}^{d}\right)$ be a test function and consider the diffeomorphism map $\left(T_{\varepsilon}\right)_{\varepsilon \in \mathbb{R}}$ in $C^{\infty}\left(\mathbb{R}^{d}, \mathbb{R}^{d}\right)$ defined by

$$
\left\{\begin{array}{l}
\frac{\partial T_{\varepsilon}}{\partial \varepsilon}=\psi \circ T_{\varepsilon}, \\
T_{0}=\mathrm{id} .
\end{array}\right.
$$

Define the probability density $G_{\varepsilon}^{x}=T_{\varepsilon \#} F_{k}^{x}$ on $\mathbb{R}^{d}$. Since $G$ satisfies $\left(H_{G}\right)$ and $T_{\varepsilon}$ is a diffeomorphism pushing $F_{k}^{x h}$ forward to $G_{\varepsilon}^{x}$, we obtain the following Monge-Kantorovich-type energy inequality:

$$
\begin{aligned}
& \frac{1}{\rho_{k}^{h}(x)}\left[\int_{\mathbb{R}^{d}} G\left(\rho_{k}^{h} G_{\varepsilon}^{x}\right) \mathrm{d} v-\int_{\mathbb{R}^{d}} G\left(\rho_{k}^{h} F_{k}^{x h}\right) \mathrm{d} v\right] \\
& \geq \int_{\mathbb{R}^{d}}\left\langle T_{\varepsilon}(v)-v, \nabla_{v}\left(G^{\prime}\left(\rho_{k}^{h} F_{k}^{x h}\right)\right)\right\rangle F_{k}^{x h} \mathrm{~d} v .
\end{aligned}
$$

Recalling the definition of $T_{\varepsilon}$, we have

$$
\left|\frac{T_{\varepsilon}(v)-v}{\varepsilon}\right| \leq\|\psi\|_{\infty}, \quad \text { for all } v \in \mathbb{R}^{d} \text { and } \varepsilon>0 .
$$

Dividing (91) by $\varepsilon>0$ and using (92) and the dominated convergence theorem, we have 


$$
\begin{aligned}
& \lim _{\varepsilon \longrightarrow 0^{+}} \frac{1}{\varepsilon \rho_{k}^{h}(x)}\left[\int_{\mathbb{R}^{d}} G\left(\rho_{k}^{h} G_{\varepsilon}^{x}\right) \mathrm{d} v-\int_{\mathbb{R}^{d}} G\left(\rho_{k}^{h} F_{k}^{x h}\right) \mathrm{d} v\right] \\
& \geq \lim _{\varepsilon \longrightarrow 0^{+}} \int_{\mathbb{R}^{d}}\left\langle\frac{T_{\varepsilon}(v)-v}{\varepsilon}, \nabla_{v}\left(G^{\prime}\left(\rho_{k}^{h} F_{k}^{x h}\right)\right)\right\rangle F_{k}^{x h} \mathrm{~d} v \\
& \geq \int_{\mathbb{R}^{d}}\left\langle\psi, \nabla_{v}\left(G^{\prime}\left(\rho_{k}^{h} F_{k}^{x h}\right)\right)\right\rangle F_{k}^{x h} \mathrm{~d} v .
\end{aligned}
$$

Furthermore, since $T_{\varepsilon}^{-1} \# G_{\varepsilon}^{x}=F_{k}^{x h}$, then the Monge-Kantorovich-type energy inequality gives

$$
\begin{aligned}
& \lim _{\varepsilon \longrightarrow 0^{+}} \frac{1}{\varepsilon \rho_{k}^{h}(x)}\left[\int_{\mathbb{R}^{d}} G\left(\rho_{k}^{h} F_{k}^{x h}\right) \mathrm{d} v-\int_{\mathbb{R}^{d}} G\left(\rho_{k}^{h} G_{\varepsilon}^{x}\right) \mathrm{d} v\right] \\
& \geq \lim _{\varepsilon \longrightarrow 0^{+}} \int_{\mathbb{R}^{d}}\left\langle\frac{T_{\varepsilon}^{-1}(v)-v}{\varepsilon}, \nabla_{v}\left(G^{\prime}\left(\rho_{k}^{h} G_{\varepsilon}^{x}\right)\right)\right\rangle G_{\varepsilon}^{x} \mathrm{~d} v \\
& =\lim _{\varepsilon \longrightarrow 0^{+}} \int_{\mathbb{R}^{d}}\left\langle\frac{v-T_{\varepsilon}(v)}{\varepsilon}, \nabla_{v} G^{\prime}\left(\left(\rho_{k}^{h} G_{\varepsilon}^{x}\right) \circ T_{\varepsilon}\right)\right\rangle F_{k}^{x} \mathrm{~d} v \\
& =-\int_{\mathbb{R}^{d}}\left\langle\psi, \nabla_{v} G^{\prime}\left(\rho_{k}^{h} F_{k}^{x h}\right)\right\rangle F_{k}^{x h} \mathrm{~d} v .
\end{aligned}
$$

This implies that

$\lim _{\varepsilon \longrightarrow 0^{+}} \frac{1}{\varepsilon \rho_{k}^{h}(x)}\left[\int_{\mathbb{R}^{d}} G\left(\rho_{k}^{h} G_{\varepsilon}^{x}\right) \mathrm{d} v-\int_{\mathbb{R}^{d}} G\left(\rho_{k}^{h} F_{k}^{x h}\right) \mathrm{d} v\right]$

$\leq \int_{\mathbb{R}^{d}}\left\langle\psi, \nabla_{v} G^{\prime}\left(\rho_{k}^{h} F_{k}^{x h}\right)\right\rangle F_{k}^{x h} \mathrm{~d} v$.

We combine now (93) and (95) to derive

$$
\begin{aligned}
& \lim _{\varepsilon \longrightarrow 0^{+}} \frac{1}{\varepsilon \rho_{k}^{h}(x)}\left[\int_{\mathbb{R}^{d}} G\left(\rho_{k}^{h} G_{\varepsilon}^{x}\right) \mathrm{d} v-\int_{\mathbb{R}^{d}} G\left(\rho_{k}^{h} F_{k}^{x h}\right) \mathrm{d} v\right] \\
& =\int_{\mathbb{R}^{d}}\left\langle\psi, \nabla_{\nu} G^{\prime}\left(\rho_{k}^{h} F_{k}^{x h}\right)\right\rangle F_{k}^{x h} \mathrm{~d} v .
\end{aligned}
$$

Since $T_{k} \# F_{k}^{x h}=G_{k}^{x h}$ and $T_{\varepsilon} \# F_{k}^{x h}=G_{\varepsilon}^{x}$, then $T_{\varepsilon} \circ T_{k}^{-1} \# G_{k}^{x h}=G_{\varepsilon}^{x}$. So,

$$
\begin{aligned}
W_{c}^{h}\left(G_{k}^{x h}, G_{\varepsilon}^{x}\right) & =\inf _{S \#_{k}^{x h}=G_{\varepsilon}^{x}} \int_{\mathbb{R}^{d}} c\left(\frac{v-S(v)}{h}\right) G_{k}^{x h} \mathrm{~d} v \\
& \leq \int_{\mathbb{R}^{d}} c\left(\frac{v-T_{\varepsilon} \circ T_{k}^{-1}(v)}{h}\right) G_{k}^{x h} \mathrm{~d} v .
\end{aligned}
$$

Thus, for $\varepsilon>0$, we have

$$
\begin{aligned}
& \frac{W_{c}^{h}\left(G_{k}^{x h}, G_{\varepsilon}^{x}\right)-W_{c}^{h}\left(G_{k}^{x h}, F_{k}^{x h}\right)}{\varepsilon} \\
& \leq \frac{1}{\varepsilon} \int_{\mathbb{R}^{d}}\left[c\left(\frac{v-T_{\varepsilon} \circ T_{k}^{-1}(v)}{h}\right)-c\left(\frac{v-T_{k}(v)}{h}\right)\right] G_{k}^{x h} \mathrm{~d} v .
\end{aligned}
$$

We use the convexity of $c$ and the fact that $T_{k} \# F_{k}^{x h}=G_{k}^{x h}$ to obtain

$$
\begin{aligned}
& \frac{h W_{c}^{h}\left(G_{k}^{x h}, G_{\varepsilon}^{x}\right)-h W_{c}^{h}\left(G_{k}^{x h}, F_{k}^{x h}\right)}{\varepsilon} \\
& \leq-\int_{\mathbb{R}^{d}}\left\langle\nabla c\left(\frac{T_{k}(v)-T_{\varepsilon}(v)}{h}\right), \frac{T_{\varepsilon}(v)-v}{\varepsilon}\right\rangle F_{k}^{x h} \mathrm{~d} v .
\end{aligned}
$$

Now, from (92) and the dominated convergence theorem, we obtained

$$
\begin{aligned}
& \lim _{\varepsilon \longrightarrow 0^{+}} \frac{h W_{c}^{h}\left(G_{k}^{x h}, G_{\varepsilon}^{x}\right)-h W_{c}^{h}\left(G_{k}^{x h}, F_{k}^{x h}\right)}{\varepsilon} \\
& \leq-\int_{\mathbb{R}^{d}}\left\langle\nabla c\left(\frac{T_{k}(v)-v}{h}\right), \psi\right\rangle F_{k}^{x h} \mathrm{~d} v .
\end{aligned}
$$

Since $F_{k}^{x h}$ minimizes the functional

$$
I(F)=\frac{1}{\rho_{k}^{h}(x)} \int_{\mathbb{R}^{d}} G\left(\rho_{k}^{h} F\right) \mathrm{d} v+h W_{c}^{h}\left(G_{k}^{x h}, F\right),
$$

on the probability space, the Euler-Lagrange equation yields

$$
\lim _{\varepsilon \longrightarrow 0^{+}} \frac{I\left(G_{\varepsilon}^{x}\right)-I\left(F_{k}^{x h}\right)}{\varepsilon}=0 .
$$

Consequently, by using (96) and (100), we have

$$
\begin{aligned}
& \int_{\mathbb{R}^{d}}\left\langle\psi, \nabla_{v} G^{\prime}\left(\rho_{k}^{h} F_{k}^{x h}\right)\right\rangle F_{k}^{x h} \mathrm{~d} v \\
& -\int_{\mathbb{R}^{d}}\left\langle\nabla c\left(\frac{T_{k}(v)-v}{h}\right), \psi\right\rangle F_{k}^{x h} \mathrm{~d} v \geq 0 .
\end{aligned}
$$

Next, by replacing $\psi$ by $-\psi$ in (96), we obtain the desired equation:

$$
\int_{\mathbb{R}^{d}}\left\langle\psi, \nabla_{v} G^{\prime}\left(\rho_{k}^{h} F_{k}^{x h}\right)\right\rangle F_{k}^{x h} \mathrm{~d} v-\int_{\mathbb{R}^{d}}\left\langle\nabla c\left(\frac{T_{k}(v)-v}{h}\right), \psi\right\rangle F_{k}^{x h} \mathrm{~d} v=0 .
$$

Thus, we conclude that

$$
\nabla c\left(\frac{T_{k}(v)-v}{h}\right)=\nabla_{v} G^{\prime}\left(\rho_{k}^{h} F_{k}^{x h}\right) a \cdot e-F_{k}^{x h} .
$$

Note that $\nabla c$ is inversible and $(\nabla c)^{-1}=\nabla c^{*}$. Then, we obtain the explicit expression of the optimal map $T_{k}$ :

$$
T_{k}(v)=v+h \nabla c^{*}\left(\nabla_{v}\left(G^{\prime}\left(\rho_{k}^{h} F_{k}^{x h}\right)\right)\right)-a \cdot e F_{k}^{x h} .
$$

The proof of this lemma is complete.

We are now ready to show that the sequence $\left(f_{k}^{h}\right)_{k}$ satisfies the time discretization (17) of the kinetic equation (1).

Let $\psi \in C_{c}^{\infty}\left(\Omega \times \mathbb{R}^{d}\right)$, then

$$
\begin{aligned}
& \int_{\Omega \times \mathbb{R}^{d}}\left(f_{k}^{h}(x, v)-f_{k-1}^{h}(x, v)\right) \psi(x, v) \mathrm{d} x \mathrm{~d} v \\
& =\int_{\Omega \times \mathbb{R}^{d}}\left(f_{k}^{h}(x, v)-g_{k-1}(x, v)\right) \psi(x, v) \mathrm{d} x \mathrm{~d} v \\
& \quad+\int_{\Omega \times \mathbb{R}^{d}}\left(g_{k}^{h}(x, v)-f_{k-1}^{h}(x, v)\right) \psi(x, v) \mathrm{d} x \mathrm{~d} v .
\end{aligned}
$$



have

By using $T_{k} \# F_{k}^{x h}=G_{k}^{x h}, f_{k}^{h}=\rho_{k}^{h} F_{k}^{x h}$, and $g_{k}^{h}=\rho_{k}^{h} G_{k}^{x h}$, we

$$
\begin{gathered}
\int_{\Omega \times \mathbb{R}^{d}}\left(f_{k}^{h}(x, v)-g_{k-1}(x, v)\right) \psi(x, v) \mathrm{d} x \mathrm{~d} v=\int_{\Omega \times \mathbb{R}^{d}}\left(\psi(x, v)-\psi\left(x, T_{k}(x)\right)\right) f_{k}^{h}(x, v) \mathrm{d} v \\
\int_{\Omega \times \mathbb{R}^{d}}\left(g_{k}^{h}(x, v)-f_{k-1}^{h}(x, v)\right) \psi(x, v) \mathrm{d} x \mathrm{~d} v=\int_{\Omega \times \mathbb{R}^{d}}(\psi(x+h v, v)-\psi(x, v)) f_{k-1}^{h} \mathrm{~d} x \mathrm{~d} v .
\end{gathered}
$$

Next, we use the Taylor formula and the expression of the optimal $T_{k}$ to obtain

$$
\begin{aligned}
& \int_{\Omega \times \mathbb{R}^{d}}\left(f_{k}^{h}(x, v)-f_{k-1}^{h}(x, v)\right) \psi(x, v) \mathrm{d} x \mathrm{~d} v \\
& =h \int_{\Omega \times \mathbb{R}^{d}}\left\langle v, \nabla_{x} \psi(x, v)\right\rangle f_{k-1}^{h}(x, v) \mathrm{d} x \mathrm{~d} v \\
& -h \int_{\Omega \times \mathbb{R}^{d}}\left\langle\nabla c^{*} \nabla_{v}\left(G^{\prime}\left(f_{k}^{h}\right)\right)\right\rangle f_{k}^{h} \mathrm{~d} x \mathrm{~d} v \\
& +h^{2} \int_{\Omega \times \mathbb{R}^{d}}\left\langle v^{\tau}, \nabla_{x}^{2} \psi(x+h \theta v, v) v\right\rangle f_{k-1}^{h} \mathrm{~d} x \mathrm{~d} v \\
& +h^{2} \int_{\Omega \times \mathbb{R}^{d}}\left\langle\sigma_{k}^{\tau}, \nabla_{v}^{2} \psi\left(x, v+\alpha h \sigma_{k}\right) \sigma_{k}\right\rangle f_{k}^{h} \mathrm{~d} x \mathrm{~d} v .
\end{aligned}
$$

Here, $\sigma_{k}=\nabla c^{*}\left(\nabla_{v}\left(G^{\prime}\left(f_{k}^{h}\right)\right)\right)$ and $\left.\theta, \alpha \in\right] 0,1[$. Now define

$$
\begin{aligned}
A_{k}^{h}[\psi]:= & \left.\frac{h^{2}}{2} \int_{\Omega \times \mathbb{R}^{d}}\left\langle\left(\sigma_{k}\right)^{\tau}, \nabla^{2} \psi\left(x, v+h \theta \sigma_{k}\right)\right) \cdot\left(\sigma_{k}\right)\right\rangle f_{k}^{h}(x, v) \mathrm{d} x \mathrm{~d} v \\
& +\frac{h^{2}}{2} \int_{\Omega \times \mathbb{R}^{d}}\left\langle v^{\tau}, \nabla_{x}^{2} \psi(x+\alpha h v, v) v\right\rangle f_{k-1}^{h} \mathrm{~d} x \mathrm{~d} v .
\end{aligned}
$$

If we show that $A_{k}^{h}[\psi]$ tends to 0 as $h$ goes to 0 , then we are done. then

Indeed, from the maximum principle, $N \leq f_{k}^{h} \leq M$, and

$$
\begin{aligned}
& \left|A_{k}^{h}[\psi]\right| \leq \frac{h^{2}}{2}\left\|\nabla_{v}^{2} \psi\right\|_{L^{\infty}\left(\Omega \times \mathbb{R}^{d}\right)} \int_{\Omega \times \mathbb{R}^{d}}\left|\nabla c^{*}\left(\nabla_{v}\left(G^{\prime}\left(f_{k}^{h}\right)\right)\right)\right|^{2} f_{k}^{h} \mathrm{~d} x \mathrm{~d} v \\
& +\frac{h^{2}}{2}\left\|\nabla_{x}^{2} \psi\right\|_{L^{\infty}\left(\Omega \times \mathbb{R}^{d}\right)}\left\|f_{0}\right\|_{L^{\infty}\left(\Omega \times \mathbb{R}^{d}\right)} \int_{K_{\psi}}|v|^{2} \mathrm{~d} x \mathrm{~d} v,
\end{aligned}
$$

where $K_{\psi}$ is a compact subset of $\Omega \times \mathbb{R}^{d}$ such that supp $\psi \subset K_{\psi}$.

Since $F_{k}^{x h}$ minimizes the general functional energy $I(F)=1 / \rho_{k}^{h} \int_{\mathbb{B}^{d}}^{k} G\left(\rho_{k}^{h} F\right) \mathrm{d} v+h W_{c}^{h}\left(F, G_{k}^{x h}\right)$ on the metric space $\left(P_{q}\left(\mathbb{R}^{d}\right), W_{c}\right)$, then

$$
\frac{1}{\rho_{k}^{h}} \int_{\mathbb{R}^{d}} G\left(\rho_{k}^{h} F_{k}^{x h}\right) \mathrm{d} v+h W_{c}^{h}\left(F_{k}^{x h}, G_{k}^{x h}\right) \leq \frac{1}{\rho_{k}^{h}} \int_{\mathbb{R}^{d}} G\left(\rho_{k}^{h} G_{k}^{x h}\right) \mathrm{d} v .
$$

We use in (112) the expression of the optimal maps $T_{k}$ and the definition of the $f_{k}^{h}$, and then, we have

$$
\begin{aligned}
& \int_{\Omega \times \mathbb{R}^{d}} G\left(f_{k-1}^{h}\right) \mathrm{d} x \mathrm{~d} v-\int_{\Omega \times \mathbb{R}^{d}} G\left(f_{k}^{h}\right) \mathrm{d} x \mathrm{~d} v \\
& \geq h \int_{\Omega \times \mathbb{R}^{d}} c\left(\nabla c^{*}\left(\nabla_{v}\left(G^{\prime}\left(f_{k}^{h}\right)\right)\right)\right) f_{k}^{h} \mathrm{~d} x \mathrm{~d} v .
\end{aligned}
$$
have

Because $G \in C^{1}((0, \infty))$ is convex and $N \leq f_{k}^{h} \leq M$, we

$$
\int_{\Omega \times \mathbb{R}^{d}} G\left(f_{k-1}^{h}\right) \mathrm{d} x \mathrm{~d} v-\int_{\Omega \times \mathbb{R}^{d}} G\left(f_{k}^{h}\right) \mathrm{d} x \mathrm{~d} v \leq G^{\prime}(M)-G^{\prime}(N) .
$$

By using (114) and the fact that $c(x) \geq A_{1}|x|^{q}$, then (113) becomes

$$
h \int_{\Omega \times \mathbb{R}^{d}}\left|\nabla c^{*}\left(\nabla_{v}\left(G^{\prime}\right)\left(f_{k}^{h}\right)\right)\right|^{q} f_{k}^{h} \mathrm{~d} x \mathrm{~d} v \leq \frac{G^{\prime}(M)-G^{\prime}(N)}{A_{1}} .
$$

Recalling (115) and (111), we obtain

$$
\begin{aligned}
& \left|A_{k}^{h}(\psi)\right| \leq h\left\|\nabla_{x}^{2} \psi\right\|_{\infty}\left|K_{\psi}\right|^{2} M \\
& \quad+h\|\| \nabla_{v}^{2} \psi \|_{\infty} 2^{q-1} \frac{G^{\prime}(M)-G^{\prime}(N)}{A_{1}}+h 2^{q-1}, \quad \text { if, } q \geq 2,
\end{aligned}
$$

$$
\begin{gathered}
\left|A_{k}^{h}(\psi)\right| \leq h\left\|\nabla_{x}^{2} \psi\right\|_{\infty}\left|K_{\psi}\right|^{2} M+h^{q-1}\left\|\nabla_{\nu}^{2} \psi\right\|_{\infty} \\
\cdot \frac{G^{\prime}(M)-G^{\prime}(N)}{A_{1}}, \quad \text { if, } q<2 .
\end{gathered}
$$

We combine (116) and (117) to conclude that $A_{k}^{h}(\psi)$ tends to 0 , when $h$ goes to 0 . Accordingly, we conclude that the sequence $\left(f_{k}^{h}\right)_{k}$ resulted from a time discretization of the kinetic equation (1).

Recalling Section 2.1, we define an approximate solution $f^{h}$ over $\left[0, \infty\left[\times \Omega \times \mathbb{R}^{d}\right.\right.$ of the kinetic equation (1) as follows:

$$
\left\{\begin{array}{l}
f^{h}(t, x, v)=f_{k}^{h}\left(x-\left(t-t_{k}\right) v, v\right), \quad \text { if } t \in\left[t_{k}, t_{k+1}\right), \\
f^{h}(0, x, v)=f_{0}(x, v)
\end{array}\right.
$$

In the next section, we establish the convergence of the sequence $\left(f^{h}\right)_{h}$ to a weak solution $f$ of (1).

\section{Convergence Results}

4.1. Weak Convergence of $\left(f^{h}\right)_{h}$. Let us consider the sequence $\left(f^{h}\right)_{h}$ as defined in (118). 
Lemma 4. Assume that $f_{0}$ satisfies $\left(H_{f_{0}}\right)$. Then, for all $k \geq 1$, we have

$$
\int_{\Omega \times \mathbb{R}^{d}}\left|f_{k}^{h}(x, v)\right|^{p} \mathrm{~d} x \mathrm{~d} v \leq \int_{\Omega \times \mathbb{R}^{d}}\left|f_{0}(x, v)\right|^{p} \mathrm{~d} x \mathrm{~d} v .
$$

Proof. Taking $H(t)=t^{p}, p>1$, in Corollary 1 , we obtain

$$
\int_{\mathbb{R}^{d}}\left|G_{k}^{x h}\right|^{p} \mathrm{~d} v-\int_{\mathbb{R}^{d}}\left|F_{k}^{x h}\right|^{p} \mathrm{~d} v \geq \int_{\mathbb{R}^{d}}\left\langle T_{k}(v)-v, \nabla_{v}\left(H \prime\left(F_{k}^{x h}\right)\right)\right\rangle F_{k}^{x h} \mathrm{~d} v,
$$

where $T_{k}$ is $c$-optimal map that pushes $F_{k}^{x h}$ forward to $G_{k}^{x h}$. We use expression of $T_{k}$ and we obtain

$$
\begin{aligned}
& \int_{\mathbb{R}^{d}}\left|G_{k}^{x h}\right|^{p} \mathrm{~d} v-\int_{\mathbb{R}^{d}}\left|F_{k}^{x h}\right|^{p} \mathrm{~d} v \\
& \geq \int_{\mathbb{R}^{d}}\left\langle T_{k}(v)-v, \nabla_{v}\left(H \prime\left(F_{k}^{x h}\right)\right)\right\rangle F_{k}^{x h} \mathrm{~d} v \\
& =p(p-1) h \int_{\mathbb{R}^{d}}\left\langle\nabla c^{*}\left(\nabla_{v} G^{\prime}\left(f_{k}^{h}\right)\right), \nabla_{v}\left(F_{k}^{x h}\right)\right\rangle\left(F_{k}^{x h}\right)^{p-1} \mathrm{~d} v .
\end{aligned}
$$

Since $\nabla_{\nu} G^{\prime}\left(f_{k}^{h}\right)=G^{\prime \prime}\left(f_{k}^{h}\right) \nabla_{v} f_{k}^{h}$ and $f_{k}^{h}=\rho_{k}^{h} F_{k}^{x h}$, becomes

$$
\begin{aligned}
& \int_{\mathbb{R}^{d}}\left|G_{k}^{x h}\right|^{p} \mathrm{~d} v-\int_{\mathbb{R}^{d}}\left|F_{k}^{x h}\right|^{p} \mathrm{~d} v \\
& \geq \frac{h p(p-1)}{\rho_{k}^{h}(x)} \int_{\mathbb{R}^{d}} \frac{\left(F_{k}^{x h}\right)^{p-1}}{G^{\prime \prime}\left(f_{k}^{h}\right)}\left\langle\nabla c^{*}\left(\nabla_{v} G^{\prime}\left(f_{k}^{h}\right)\right), \nabla_{v} G^{\prime}\left(f_{k}^{h}\right)\right\rangle \mathrm{d} v .
\end{aligned}
$$

From the convexity of $c$ and $c^{*}$, we have

$$
a \cdot b=c(a)+c^{*}(b), \quad \text { if } a, b \in \mathbb{R}^{d} \text {, with } a=\nabla c^{*}(b) .
$$

Thus, using (123) with $a=\nabla c^{*}\left(\nabla_{v}\left(G^{\prime}\left(f_{k}^{h}\right)\right)\right)$ and $b=\nabla_{v} G^{\prime}\left(f_{k}^{h}\right)$, (122) becomes

$$
\int_{\mathbb{R}^{d}}\left|G_{k}^{x h}\right|^{p} \mathrm{~d} v-\int_{\mathbb{R}^{d}}\left|F_{k}^{x h}\right|^{p} \mathrm{~d} v \geq \frac{h p(p-1)}{\rho_{k}^{h}(x)} \int_{\mathbb{R}^{d}} \frac{\left(F_{k}^{x h}\right)^{p-1}}{G^{\prime \prime}\left(f_{k}^{h}\right)} c\left(\nabla c^{*}\left(\nabla_{v} G^{\prime}\left(f_{k}^{h}\right)\right)\right) \mathrm{d} v+\frac{h p(p-1)}{\rho_{k}^{h}(x)} \int_{\mathbb{R}^{d}} \frac{\left(F_{k}^{x h}\right)^{p-1} G^{\prime \prime}\left(f_{k}^{h}\right)}{c^{*}}\left(\nabla_{v} G^{\prime}\left(f_{k}^{h}\right)\right) \mathrm{d} v .
$$

Since $c \geq 0$ and $c(0)=0$, then $c^{*} \geq 0$; hence,

$$
\int_{\mathbb{R}^{d}}\left|G_{k}^{x h}\right|^{p} \mathrm{~d} v-\int_{\mathbb{R}^{d}}\left|F_{k}^{x h}\right|^{p} \mathrm{~d} v \geq 0
$$

Multiplying (125) by $\left|\rho_{k}^{h}\right|^{p}$, we obtain after integration

$$
\int_{\Omega \times \mathbb{R}^{d}}\left|f_{k}^{h}\right|^{p} \mathrm{~d} x \mathrm{~d} v \leq \int_{\Omega \times \mathbb{R}^{d}}\left|g_{k}^{h}\right|^{p} \mathrm{~d} x \mathrm{~d} v=\int_{\Omega \times \mathbb{R}^{d}}\left|f_{k-1}^{h}\right|^{p} \mathrm{~d} x \mathrm{~d} v
$$

and then by an iteration process on $k$, we get the proof of Lemma 4.
Lemma 5. Assume that $f_{0} \in L^{p}\left(\Omega \times \mathbb{R}^{d}\right) \cap P_{q}\left(\Omega \times \mathbb{R}^{d}\right)$ with $\int_{\Omega \times \mathbb{R}^{d}} G\left(f_{0}\right) d x d v<\infty$ and $0<N \leq f_{0} \leq M$. For $0<T<\infty$, we have

$$
\int_{[0, T] \times \Omega \times \mathbb{R}^{d}}\left|f^{h}(t, x, v)\right|^{p} \mathrm{~d} t \mathrm{~d} x \mathrm{~d} v \leq T \int_{\Omega \times \mathbb{R}^{d}}\left|f_{0}(x, v)\right|^{p} \mathrm{~d} x \mathrm{~d} v .
$$

Proof. Let $0<T<\infty$ and let $h>0$ be a step such that $T / h \in \mathbb{N}^{*}$.

We use Lemma 3 and the definition of $f^{h}$ in (118) to obtain

$$
\int_{[0, T] \times \Omega \times \mathbb{R}^{d}}\left|f^{h}(t, x, v)\right|^{p} \mathrm{~d} t \mathrm{~d} x \mathrm{~d} v=\sum_{k=1}^{T / h} h \int_{\Omega \times \mathbb{R}^{d}}\left|f_{k}^{h}\right|^{p} \mathrm{~d} x \mathrm{~d} v \leq T \int_{\Omega \times \mathbb{R}^{d}}\left|f_{0}(x, v)\right|^{p} \mathrm{~d} x \mathrm{~d} v<\infty .
$$

4.2. Weak Convergence of the Linear Term. Here, we study the weak convergence of the linear term.

Proposition 3. Assume that $f_{0}$ satisfy $\left(H_{f_{0}}\right)$. Then, there exists a function $f$ in $L^{p}\left([0,] \times \Omega \times \mathbb{R}^{d}\right) \cap L^{\infty}([0, T] \times \Omega \times$ $\left.\mathbb{R}^{d}\right)$ until a subsequence $\left(f^{h}\right)_{h}$ converges to $f$ weakly in $L^{p}\left([0, T] \times \Omega \times \mathbb{R}^{d}\right)$ and weakly-* in $L^{\infty}\left([0, T] \times \Omega \times \mathbb{R}^{d}\right)$.
Moreover,

$$
\begin{gathered}
\lim _{k \rightarrow \infty} \int_{[0, T] \times \Omega \times \mathbb{R}^{d}}\left[\partial_{t} \psi+v \cdot \nabla_{x} \psi\right] f^{h_{k}} \mathrm{~d} t \mathrm{~d} x \mathrm{~d} v \\
\quad=\int_{[0, T] \times \Omega \times \mathbb{R}^{d}}\left[\partial_{t} \psi+v \cdot \nabla_{x} \psi\right] f \mathrm{~d} t \mathrm{~d} x \mathrm{~d} v,
\end{gathered}
$$


for every test function $\psi \in C_{c}^{\infty}\left(\mathbb{R} \times \Omega \times \mathbb{R}^{d}\right)$, with $\sup p \psi(., x, v) \subset[-T, T]$.

Proof. Since $f_{0} \in L^{p}\left(\Omega \times \mathbb{R}^{d}\right) \cap L^{\infty}\left(\Omega \times \mathbb{R}^{d}\right)$ (see Proposition 2 and Lemma 4), then

$$
\begin{aligned}
\left\|f^{h}\right\|_{L^{\infty}} & \leq\left\|f_{0}\right\|_{L^{\infty}}\left(\Omega \times \mathbb{R}^{d}\right) \\
\left\|f^{h}\right\|_{L^{p}} & \leq T^{1 / p}\left\|f_{0}\right\|_{L^{p}\left(\Omega \times \mathbb{R}^{d}\right)} .
\end{aligned}
$$

Hence, the sequence $\left(f^{h}\right)_{h}$ is bounded in $L^{p}\left([0, T] \times \Omega \times \mathbb{R}^{d}\right) \cap L^{\infty}\left([0, T] \times \Omega \times \mathbb{R}^{d}\right)$, and then, there is subsequence of $\left(f^{h}\right)_{h}$ still denoted $\left(f^{h}\right)_{h}$ that converges to a nonnegative function $f$ weakly in $L^{p}\left([0, T] \times \Omega \times \mathbb{R}^{d}\right)$ and weakly-* in $L^{\infty}[0, T] \times \Omega \times \mathbb{R}^{d}$.

Since $\partial_{t} \psi+v \cdot \nabla_{x} \psi \in L^{q}\left([0, T] \times \Omega \times \mathbb{R}^{d}\right)$, for every $\psi \in C_{c}^{\infty}\left(\mathbb{R} \times \Omega \times \mathbb{R}^{d}\right)$ such that $\sup p \psi(., x, v) \subset[-T, T]$, we finally obtain Proposition 3.

4.3. Weak Convergence of the Nonlinear Term. In this section, we establish the weak convergence of the nonlinear term.

First, we prove that the sequence $\nabla_{\nu} G^{\prime}\left(f^{h}\right)$ is bounded in $L^{p}\left([0, T] \times \Omega \times \mathbb{R}^{d}\right)$, and that $\nabla c^{*}\left(\nabla_{v}\left(G^{\prime}\left(f^{h}\right)\right)\right)$ is bounded in $L^{q}\left([0, T] \times \Omega \times \mathbb{R}^{d}\right)$ where $0<T<\infty$.

Lemma 6. Assume that $f_{0}, c$, and $G$ satisfy, respectively, $\left(H_{f_{0}}\right),\left(H_{c}\right)$, and $\left(H_{G}\right)$. Then, for all $0<T<\infty$ fixed,

$$
\begin{aligned}
& \left.\int_{[0, T] \times \Omega \times \mathbb{R}^{d}}|| \nabla c^{*}\left(\nabla_{v}\left(G^{\prime}\left(f^{h}\right)\right)\right)\right|^{q} \mathrm{~d} t \mathrm{~d} x \mathrm{~d} v \\
& \leq \frac{T}{A_{1} N}\left[G^{\prime}(M)-G^{\prime}(N)\right], \\
& \left.\int_{[0, T] \times \Omega \times \mathbb{R}^{d}}|| \nabla_{v}\left(G^{\prime}\left(f^{h}\right)\right)\right|^{p} \mathrm{~d} t \mathrm{~d} x \mathrm{~d} v \\
& \leq\left[A_{2} T+G^{\prime}(M)-G^{\prime}(N)\right] \frac{p q^{p-1} A_{2}^{p-1}}{N} .
\end{aligned}
$$

Proof. Using (115), we have

$$
\begin{gathered}
\int_{\Omega \times \mathbb{R}^{d}}\left|\nabla c^{*}\left(\nabla_{v}\left(G^{\prime}\left(f_{k}^{h}\right)\right)\right)\right|^{q} f_{k}^{h} \mathrm{~d} x \mathrm{~d} v \\
\leq \frac{G^{\prime}(M)-G^{\prime}(N)}{A_{1}} .
\end{gathered}
$$

But $f^{h} \geq N$, and then

$$
\begin{gathered}
\int_{[0, T] \times \Omega \times \mathbb{R}^{d}}\left|\nabla c^{*}\left(\nabla_{v}\left(G^{\prime}\left(f^{h}\right)\right)\right)\right|^{q} \mathrm{~d} t \mathrm{~d} x \mathrm{~d} v \\
\leq\left[G^{\prime}(M)-G^{\prime}(N)\right]^{\prime} \frac{T}{N A_{1}} .
\end{gathered}
$$

Recalling (123), with $a=\nabla c^{*}\left(\nabla_{v}\left(G^{\prime}\left(f^{h}\right)\right)\right)$ and $b=\nabla_{v} G^{\prime}\left(f^{h}\right)$, we have

$$
\begin{aligned}
& \nabla c^{*}\left(\nabla_{v}\left(G^{\prime}\left(f^{h}\right)\right)\right) \cdot \nabla_{v} G^{\prime}\left(f^{h}\right) \\
& \quad-c\left(\nabla c^{*}\left(\nabla_{v}\left(G^{\prime}\left(f^{h}\right)\right)\right)\right)=c^{*}\left(\nabla_{v} G^{\prime}\left(f^{h}\right)\right),
\end{aligned}
$$

with $c \geq 0$

Then,

$$
\begin{aligned}
& \int_{[0, T] \times \Omega \times \mathbb{R}^{d}} c^{*}\left(\nabla_{v}\left(G^{\prime}\left(f^{h}\right)\right)\right) f^{h} \mathrm{~d} t \mathrm{~d} x \mathrm{~d} v \\
& \leq \int_{[0, T] \times \Omega \times \mathbb{R}^{d}} \nabla c^{*}\left(\nabla_{v}\left(G^{\prime}\left(f^{h}\right)\right)\right) \nabla_{v} G^{\prime}\left(f^{h}\right) \mathrm{d} t \mathrm{~d} x \mathrm{~d} v \\
& =\sum_{k=1}^{T / h} h \int_{\Omega \times \mathbb{R}^{d}} \nabla c^{*}\left(\nabla_{v}\left(G^{\prime}\left(f_{k}^{h}\right)\right)\right) \nabla_{v} G^{\prime}\left(f_{k}^{h}\right) \mathrm{d} x \mathrm{~d} v \\
& \leq \sum_{k=1}^{T / h}\left[\int_{\Omega \times \mathbb{R}^{d}} G\left(f_{k-1}^{h}\right) \mathrm{d} x \mathrm{~d} v-\int_{\Omega \times \mathbb{R}^{d}} G\left(f_{k}^{h}\right) \mathrm{d} x \mathrm{~d} v\right] .
\end{aligned}
$$

Moreover,

$$
\begin{aligned}
& \sum_{k=1}^{T / h}\left[\int_{\Omega \times \mathbb{R}^{d}} G\left(f_{k-1}^{h}\right) \mathrm{d} x \mathrm{~d} v-\int_{\Omega \times \mathbb{R}^{d}} G\left(f_{k}^{h}\right) \mathrm{d} x \mathrm{~d} v\right] \\
& =\int_{\Omega \times \mathbb{R}^{d}} G\left(f_{0}\right) \mathrm{d} x \mathrm{~d} v-\int_{\Omega \times \mathbb{R}^{d}} G\left(f_{T / h}\right) \mathrm{d} x \mathrm{~d} v \\
& \leq G^{\prime}(M)-G^{\prime}(N),
\end{aligned}
$$

and then,

$$
\int_{[0, T] \times \Omega \times \mathbb{R}^{d}} c^{*}\left(\nabla_{v}\left(G^{\prime}\left(f^{h}\right)\right)\right) f^{h} \mathrm{~d} t \mathrm{~d} x \mathrm{~d} v \leq G^{\prime}(M)-G^{\prime}(N) .
$$

Because $\quad c(y) \leq A_{2}|y|^{q}+A_{2}$, we have $x \cdot y-c(y) \geq x \cdot y-A_{2}|y|^{q}-A_{2}$ for all $x, y \in \mathbb{R}^{d}$ and then

$$
c^{*}(x):=\sup _{y \in \mathbb{R}^{d}}\{x \cdot y-c(y)\} \geq-A_{2}+\frac{|x|^{p}}{p q^{p-1} A_{2}^{p-1}} \text {. }
$$

Consequently,

$$
\begin{aligned}
& -A_{2} T+\frac{1}{p q^{p-1} A_{2}^{p-1}} \int_{[0, T] \times \Omega \times \mathbb{R}^{d}}\left|\nabla_{v}\left(G^{\prime}\left(f^{h}\right)\right)\right|^{p} f^{h} \mathrm{~d} t \mathrm{~d} x \mathrm{~d} v \\
& \leq G^{\prime}(M)-G^{\prime}(N) .
\end{aligned}
$$

Using $f^{h} \geq N$, we obtain

$$
\begin{aligned}
& \int_{[0, T] \times \Omega \times \mathbb{R}^{d}}\left|\nabla_{v}\left(G^{\prime}\left(f^{h}\right)\right)\right|^{p} \mathrm{~d} t \mathrm{~d} x \mathrm{~d} v \\
& \leq\left[T A_{2}+G^{\prime}(M)-G^{\prime}(N)\right] \frac{p q^{p-1} A_{2}^{p-1}}{N} .
\end{aligned}
$$

4.4. Strong Convergence of $\left(f^{h}\right)_{h}$. In order to prove the strong convergence result, we need to establish the following compactness results for $\left(f^{h}\right)_{h}$ in $L^{p}\left([0, T] \times \Omega \times \mathbb{R}^{d}\right)$. 
Lemma 7 (velocity-compactness). Assume that $f_{0}$ satisfies $\left(H_{f_{0}}\right)$. Fix $0<T<\infty$ and $0 \neq \eta \in \mathbb{R}^{d}$ such that $|\eta|$ is small. Then,

$$
\int_{[0, T] \times \Omega \times \mathbb{R}^{d}}\left|f^{h}(t, x, v+\eta)-f^{h}(t, x, v)\right|^{p} \mathrm{~d} t \mathrm{~d} x \mathrm{~d} v \leq|\eta|^{p} K,
$$

where $\quad K:=\inf _{t \in[N, M]} 1 /\left|G^{\prime \prime}(t)\right|^{p}\left[T A_{2}+G^{\prime}(M)-G^{\prime}(N)\right]$ $\left(p q^{p-1} A_{2}^{p-1} / N\right)$ is a constant.

Proof. We use $\nabla_{v} G^{\prime}\left(f^{h}\right)=G^{\prime \prime}\left(f^{h}\right) \nabla_{v} f^{h}, N \leq f^{h} \leq M$, and (140) to produce that

$$
\int_{[0, T] \times \Omega \times \mathbb{R}^{d}}\left|\nabla_{v} f^{h}\right|^{p} \mathrm{~d} t \mathrm{~d} x \mathrm{~d} v \leq K .
$$

Then, $f^{h}$ and $\nabla_{v} f^{h} \in L^{p}\left([0, T] \times \Omega \times \mathbb{R}^{d}\right)$ and approximating $f^{h}(t, x,$.$) by C_{c}^{\infty}\left(\mathbb{R}^{d}\right)$ functions in $W^{1, p}\left(\mathbb{R}^{d}\right)$, we obtain

$$
\begin{aligned}
& \int_{[0, T] \times \Omega \times \mathbb{R}^{d}}\left|f^{h}(t, x, v+\eta)-f^{h}(t, x, v)\right|^{p} \mathrm{~d} t \mathrm{~d} x \mathrm{~d} v \\
& \leq|\eta|^{p} \int_{[0, T] \times \Omega \times \mathbb{R}^{d}}\left|\left(\nabla_{v} f^{h}\right)\right|^{p} \mathrm{~d} t \mathrm{~d} x \mathrm{~d} v .
\end{aligned}
$$

This implies that

$$
\int_{[0, T] \times \Omega \times \mathbb{R}^{d}}\left|f^{h}(t, x, v+\eta)-f^{h}(t, x, v)\right|^{p} \mathrm{~d} t \mathrm{~d} x \mathrm{~d} v \leq|\eta|^{p} K .
$$

Lemma 8 (position-compactness). Assume that $f_{0}$ satisfies $\left(H_{f_{0}}\right)$. We fix $0<T<\infty$ and $0 \neq \eta \in \mathbb{R}$ such that $|\eta|$ is small. Then,

$\int_{[0, T] \times \Omega \times \mathbb{R}^{d}}\left|f^{h}(t, x+h \eta v, v)-f^{h}(t, x, v)\right|^{p} \mathrm{~d} t \mathrm{~d} x \mathrm{~d} v \leq|\eta|^{p} T K_{1}$,

where $K_{1}=2^{p}\left\|f_{0}\right\|_{L^{p}\left(\Omega \times \mathbb{R}^{d}\right)}^{p}$.

Proof. By fixing $v$, (17) becomes

$$
f_{k}^{h}-f_{k-1}^{h}+h v \cdot \nabla_{x} f_{k}^{h}=o(h),
$$

where $o(h)$ tends to 0 when $h$ goes to 0 . We use (146), and the fact that $\left(f_{k}^{h}\right)_{k}$ is bounded in $L^{p}\left(\Omega \times \mathbb{R}^{d}\right)$ to deduce that $\left(h v \cdot \nabla_{x} f_{k}^{h}\right)_{k}$ is bounded in $L^{p}\left(\Omega \times \mathbb{R}^{d}\right)$. Then, by using Lemma 4 , we have

$$
\left\|h v \cdot \nabla_{x} f_{k}^{h}\right\|_{L^{p}\left(\Omega \times \mathbb{R}^{d}\right)} \leq 2\left\|f_{0}\right\|_{L^{p}\left(\Omega \times \mathbb{R}^{d}\right)} .
$$

Thus, $f^{h}(t, ., v) \in L^{p}(\Omega)$, and approximating $f^{h}(t, ., v)$ by $C_{c}^{\infty}\left(\mathbb{R}^{d}\right)$ functions, we use the fact that $\left(h v \cdot \nabla_{x} f_{k}^{h}\right)_{k}$ is bounded in $L^{p}\left(\Omega \times \mathbb{R}^{d}\right)$ to deduce that

$$
\begin{aligned}
& \int_{[0, T] \times \Omega \times \mathbb{R}^{d}}\left|f^{h}(t, x+h \eta v, v)-f^{h}(t, x, v)\right|^{p} \mathrm{~d} t \mathrm{~d} x \mathrm{~d} v \\
& \leq|\eta|^{p} \int_{[0, T] \times \Omega \times \mathbb{R}^{d}}\left|h v \cdot \nabla_{x} f^{h}(t, x, v)\right|^{p} \mathrm{~d} t \mathrm{~d} x \mathrm{~d} v \\
& \leq|\eta|^{p} 2^{p} T\left(\int_{\Omega \times \mathbb{R}^{d}}\left|f_{0}(x, v)\right|^{p} \mathrm{~d} x \mathrm{~d} v\right) .
\end{aligned}
$$

Lemma 9. Assume that $\left(H_{f_{0}}\right)$ holds. Let $\tau>0$ be quite small such that $[t, t+\tau] \subset[0, T], \forall t \in[0, T]$, where $0<T<\infty$ is fixed. We have

$$
\begin{aligned}
& \int_{[0, T] \times \Omega \times \mathbb{R}^{d}}\left[G^{\prime}\left(f^{h}(t+\tau)\right)-G^{\prime}\left(f^{h}(t)\right)\right]\left[f^{h}(t+\tau)-f^{h}(t)\right] \mathrm{d} t \mathrm{~d} x \mathrm{~d} v \\
& \leq \tau K_{2}\left(\Omega, T, N, M, f_{0}, G^{\prime}, q, p\right),
\end{aligned}
$$

where $K_{2}\left(\Omega, T, N, M, f_{0}, G^{\prime}, q, p\right)$ is a positive constant depending on $\Omega, T, N, M, f_{0}, G^{\prime}, q, p$.

Proof. Choose $h>0$ such that $T / h \in \mathbb{N}^{*}$ and $\tau / h=N \in \mathbb{N}^{*}$. Define

$$
\begin{aligned}
A= & \int_{[0, T] \times \Omega \times \mathbb{R}^{d}}\left[G^{\prime}\left(f^{h}(t+\tau)\right)-G^{\prime}\left(f^{h}(t)\right)\right] \\
& \cdot\left[f^{h}(t+\tau)-f^{h}(t)\right] \mathrm{d} t \mathrm{~d} x \mathrm{~d} v .
\end{aligned}
$$

Using the definition of $f^{h}$ in (118), we obtain

$$
A=\sum_{k=1}^{T / h} h \int_{\Omega \times \mathbb{R}^{d}}\left[\psi_{h, k}(x, v)\right]\left[f_{k-1+N}^{h}(x, v)-f_{k-1}^{h}(x, v)\right] \mathrm{d} x \mathrm{~d} v,
$$

where $\psi_{h, k}(x, v)=\left[G^{\prime}\left(f_{k-1+N}^{h}(x, v)\right)-G^{\prime}\left(f_{k-1}^{h}(x, v)\right)\right]$.

Note that

$$
f_{k-1+N}^{h}(x, v)-f_{k-1}^{h}(x, v)=\sum_{i=0}^{N-1}\left[f_{k-1+N-i}^{h}(x, v)-f_{k-2+N-i}^{h}(x, v)\right] .
$$

Using (152), we have

$$
\begin{aligned}
A & =\sum_{k=1}^{T / h} \sum_{i=0}^{N-1} h \int_{\Omega \times \mathbb{R}^{d}}\left[\psi_{h, k}(x, v)\right]\left[f_{k-1+N-i}^{h}(x, v)-f_{k-2+N-i}^{h}(x, v)\right] \mathrm{d} x \mathrm{~d} v \\
& =C+D,
\end{aligned}
$$

where

$$
\begin{aligned}
C & =\sum_{k=1}^{T / h} \sum_{i=0}^{N-1} h \int_{\Omega \times \mathbb{R}^{d}}\left[\psi_{h, k}(x, v)\right]\left[f_{k-1+N-i}^{h}(x, v)-g_{k-1+N-i}^{h}(x, v)\right] \mathrm{d} x \mathrm{~d} v, \\
D & =\sum_{k=1}^{T / h} \sum_{i=0}^{N-1} h \int_{\Omega \times \mathbb{R}^{d}}\left[\psi_{h, k}(x, v)\right]\left[g_{k-1+N-i}^{h}(x, v)-f_{k-2+N-i}^{h}(x, v)\right] \mathrm{d} x \mathrm{~d} v .
\end{aligned}
$$

Using $S_{k, N}^{h, i}$ as a $c$-optimal map that pushes $F_{k-1+N-i}^{x h}$ forward to $G_{k-1+N-i}^{x h}$, we have

$$
C=\sum_{k=1}^{T / h} \sum_{i=0}^{N-1} h \int_{\Omega \times \mathbb{R}^{d}}\left[\psi_{h, k}(x, v)-\psi_{h, k}\left(x, S_{k, N}^{h, i}(v)\right)\right] f_{k-1+N-i}^{h}(x, v) \mathrm{d} x \mathrm{~d} v .
$$

On the contrary, $\nabla_{v} G^{\prime}\left(f_{k-1+N-i}^{h}\right) \in L^{p}\left(\Omega \times \mathbb{R}^{d}\right)$ (see Lemma 6), and from assumptions on $G, G^{\prime}(s) / s$ is continuous on $\mathbb{R}^{*}$, and then there exists a constant $K$ such that $G^{\prime}(s) \leq K \cdot s$ for $0<N \leq s \leq M$. Since $0<N \leq f_{k-1+N-i}^{h} \leq M$ (see Lemma 2) and $f_{k-1+N-i}^{h} \in L^{p}\left(\Omega \times \mathbb{R}^{d}\right)$, then $G^{\prime}\left(f_{k-1+N-i}^{h}\right) \in L^{p}\left(\Omega \times \mathbb{R}^{d}\right)$. So $G^{\prime}\left(f_{k-1+N-i}^{h}(x,).\right) \in$ $W^{1, p}\left(\mathbb{R}^{d}\right)$. 
Approximating $G^{\prime}\left(f_{k-1+N-i}^{h}(x,).\right)$ by $C_{c}^{\infty}\left(\mathbb{R}^{d}\right)$ functions and using the dominated convergence theorem, we have

$$
\left.C=\sum_{i=1}^{N-1} \sum_{k=1}^{T / h} h \int_{\Omega \times \mathbb{R}^{d}} f_{k-1+N-i}^{h}(x, v)\left\langle v-S_{k, N}^{h, i}(x, v), \nabla_{v} \psi_{h, k}\left(x, v+\theta\left(v-S_{k, N}^{h, i}(x, v)\right)\right)\right\rangle \mathrm{d} x \mathrm{~d} v, \quad \text { for } \theta \in\right] 0,1[\text {. }
$$

Note

$v-S_{k, N}^{h, i}=-h \nabla c^{*}\left(\nabla_{v} G^{\prime}\left(f_{k-1+N-i}^{h}\right)\right) \in L^{q}\left(\Omega \times \mathbb{R}^{d}\right)$ that and $\nabla_{v} \psi_{h, k}=\nabla_{v} G^{\prime}\left(f_{k-1+N}^{h}\right)-\nabla_{v} G^{\prime}\left(f_{k-1}^{h}\right) \in L^{p}\left(\Omega \times \mathbb{R}^{d}\right)$ Lemma 6).

So, by using Hölder's inequality, we have that

$$
\begin{aligned}
& C \leq \sum_{i=1}^{N-1} \sum_{k=1}^{T / h} h\left(\int_{\Omega \times \mathbb{R}^{d}}\left|v-S_{k, N}^{h, i}(v)\right|^{q} f_{k-1+N-i}^{h}(x, v) \mathrm{d} x \mathrm{~d} v\right)^{1 / q}\left(\int_{\Omega \times \mathbb{R}^{d}}\left|\nabla_{v} \psi_{h, k}\right|^{p}\right)^{1 / p} \\
& \leq \sum_{i=1}^{N-1} \sum_{k=1}^{T / h} \frac{h}{A_{1}^{1 / q}}\left(\int_{\Omega} \rho_{k-1+N-i}^{h}(x) W_{c}^{h}\left(F_{k-1+N-i}^{x h}, G_{k-1+N-i}^{x h}\right) \mathrm{d} x\right)^{1 / q}\left(\int_{\Omega \times \mathbb{R}^{d}}\left|\nabla_{v} \psi_{h, k}\right|^{p}\right)^{1 / p} .
\end{aligned}
$$

Using the algorithm defined in Section 2.1 and Lemma 6,

Thus, we have

$$
\begin{gathered}
\int_{\Omega} \rho_{k-1+N-i}^{h}(x) W_{c}^{h}\left(F_{k-1+N-i}^{x h}, G_{k-1+N-i}^{x h}\right) \mathrm{d} x \\
\leq h^{q-1}\left[\int_{\Omega \times \mathbb{R}^{d}} G\left(f_{k-2+N-i}^{h}(x, v)\right) \mathrm{d} x \mathrm{~d} v\right. \\
\left.\quad-\int_{\Omega \times \mathbb{R}^{d}} G\left(f_{k-1+N-i}^{h}(x, v)\right) \mathrm{d} x \mathrm{~d} v\right] .
\end{gathered}
$$

$$
\begin{aligned}
& C \leq \sum_{i=1}^{N-1} \sum_{k=1}^{T / h} \frac{h}{A_{1}^{1 / q}}\left[\int_{\Omega \times \mathbb{R}^{d}} G\left(f_{k-2+N-i}^{h}(x, v)\right) \mathrm{d} x \mathrm{~d} v-\int_{\Omega \times \mathbb{R}^{d}} G\left(f_{k-1+N-i}^{h}(x, v)\right) \mathrm{d} x \mathrm{~d} v\right]^{1 / q} \times \\
& h^{(q-1) / q}\left(\int_{\Omega \times \mathbb{R}^{d}}\left|\nabla_{v} \psi_{h, k}\right|^{p}\right)^{1 / p} .
\end{aligned}
$$

Applying the Hölder's inequality in the previous relation yields

$$
\begin{aligned}
& C \leq \frac{1}{A_{1}^{1 / q}} h^{1-(1 / p)} h^{(q-1) / q}\left\{\sum_{k=1}^{T / h}\left[\int_{\Omega \times \mathbb{R}^{d}} G\left(f_{k-2+N-i}^{h}(x, v)\right) \mathrm{d} x \mathrm{~d} v-\int_{\Omega \times \mathbb{R}^{d}} G\left(f_{k-1+N-i}^{h}(x, v)\right) \mathrm{d} x \mathrm{~d} v\right]\right\}^{1 / q} \times \\
& \sum_{i=1}^{N-1}\left(\sum_{k=1}^{T / h} h \int_{\Omega \times \mathbb{R}^{d}}\left|\nabla_{v} \psi_{h, k}\right|^{p}\right)^{1 / p} .
\end{aligned}
$$

We notice that 


$$
\begin{aligned}
& \sum_{k=1}^{T / h}\left[\int_{\Omega \times \mathbb{R}^{d}} G\left(f_{k-2+N-i}^{h}(x, v)\right) \mathrm{d} x \mathrm{~d} v\right. \\
& \left.\quad-\int_{\Omega \times \mathbb{R}^{d}} G\left(f_{k-1+N-i}^{h}(x, v)\right) \mathrm{d} x \mathrm{~d} v\right] \\
& =\int_{\Omega \times \mathbb{R}^{d}} G\left(f_{-1+N-i}^{h}(x, v)\right) \mathrm{d} x \mathrm{~d} v \\
& \quad-\int_{\Omega \times \mathbb{R}^{d}} G\left(f_{(T / h)-1+N-i}^{h}(x, v)\right) \mathrm{d} x \mathrm{~d} v \leq\left[G^{\prime}(M)-G^{\prime}(N)\right],
\end{aligned}
$$

Next, we use Lemma 6, and we have

$$
\begin{aligned}
& \left(\sum_{k=1}^{T / h} h \int_{\Omega \times \mathbb{R}^{d}}\left|\nabla_{v} \psi_{h, k}\right|^{p}\right)^{1 / p}=\left(\sum_{k=1}^{T / h} h \int_{\Omega \times \mathbb{R}^{d}}|| \nabla_{v} G^{\prime}\left(f_{k-1+N}^{h}\right)-\left.\nabla_{v} G^{\prime}\left(f_{k-1}^{h}\right)\right|^{p}\right)^{1 / p} \\
& \left.\leq\left. 2^{(p-1) / p}\left(\sum_{k=1}^{T / h} h \int_{\Omega \times \mathbb{R}^{d}} \mid \nabla_{v} G^{\prime}\left(f_{k-1+N}^{h}\right)\right)\right|^{p}+\sum_{k=1}^{T / h} h \int_{\Omega \times \mathbb{R}^{d}}\left|\nabla_{\nu} G^{\prime}\left(f_{k-1}^{h}\right)\right|^{p}\right)^{1 / p} \\
& \leq 2^{(p-1) / p}\left(2 M_{0}(T, \Omega)\right)^{1 / p}=2\left(M_{0}(T, \Omega)\right)^{1 / p} .
\end{aligned}
$$

We combine (160), (161), and (162) to get

$$
\begin{aligned}
C & \leq \sum_{i=1}^{N-1} 2 h^{1-(1 / p)} h^{(q-1) / q}\left[G^{\prime}(M)-G^{\prime}(N)\right]^{1 / q}\left(M_{0}(T, \Omega)\right)^{1 / p} \\
& \leq N h^{2-((1 / p)+(1 / q))}\left[G^{\prime}(M)-G^{\prime}(N)\right]^{1 / q}\left(M_{0}(T, \Omega)\right)^{1 / p} .
\end{aligned}
$$

Writing $N h=\tau$, we then have

$$
C \leq\left[G^{\prime}(M)-G^{\prime}(N)\right]^{1 / q}\left(M_{0}(T, \Omega)\right)^{1 / p} \tau .
$$

On the contrary,

$$
\begin{aligned}
D= & \sum_{k=1}^{T / h} \sum_{i=0}^{N-1} h \int_{\Omega \times \mathbb{R}^{d}}\left[\psi_{h, k}(x, v)\right] \\
& {\left[f_{k-2+N-i}^{h}(x, v)-g_{k-1+N-i}^{h}(x, v)\right] \mathrm{d} x \mathrm{~d} v } \\
= & \sum_{k=1}^{T / h} \sum_{i=0}^{N-1} h \int_{\Omega \times \mathbb{R}^{d}}\left[\psi_{h, k}(x, v)\right] \\
& {\left[f_{k-2+N-i}^{h}(x, v)-f_{k-2+N-i}^{h}(x-h v, v)\right] \mathrm{d} x \mathrm{~d} v . }
\end{aligned}
$$

Since $\quad G^{\prime}\left(f_{k-1+N-i}^{h}(., v)\right) \in W^{1, p}(\Omega) \quad$ and then $\psi_{h, k}(., v) \in W^{1, p}(\Omega)$, we approximate $\psi_{h, k}(., v)$ by $C_{c}^{\infty}(\Omega)$ functions and we get

$$
D=-\sum_{k=1}^{T / h} \sum_{i=0}^{N-1} h^{2} \int_{\Omega \times \mathbb{R}^{d}} v \cdot \nabla_{x} \psi_{h, k}(x, v) f_{k-2+N-i}^{h}(x, v) \mathrm{d} x \mathrm{~d} v .
$$

Note that $v \cdot \nabla_{x}\left(G^{\prime}\left(f_{k}^{h}\right)\right)=G^{\prime \prime}\left(f_{k}^{h}\right) v \cdot \nabla_{x} f_{k}^{h}$, and according to Lemma $8, v \cdot \nabla_{x} f_{k}^{h} \in L^{p}\left(\Omega \times \mathbb{R}^{d}\right)$. So, since $G^{\prime \prime}$ is continuous and $N \leq f_{k}^{h} \leq M$, we have $\left(v \cdot \nabla_{x} G^{\prime}\left(f_{k}^{h}\right)\right)_{h, k} \in L^{p}\left(\Omega \times \mathbb{R}^{d}\right)$.

Consequently,

$$
\begin{aligned}
D= & -\sum_{k=1}^{T / h} \sum_{i=0}^{N-1} h^{2} \int_{\Omega \times \mathbb{R}^{d}} v \cdot \nabla_{x} \psi_{h, k}(x, v) f_{k-2+N-i}^{h}(x, v) \mathrm{d} x \mathrm{~d} v \\
= & -\sum_{k=1}^{T / h} \sum_{i=0}^{N-1} h^{2} \int_{\Omega \times \mathbb{R}^{d}} v \cdot \nabla_{x} G^{\prime}\left(f_{k-1+N}^{h}\right)(x, v) f_{k-2+N-i}^{h} \mathrm{~d} x \mathrm{~d} v \\
& +\sum_{k=1}^{T / h} \sum_{i=0}^{N-1} h^{2} \int_{\Omega \times \mathbb{R}^{d}} v \cdot \nabla_{x} G^{\prime}\left(f_{k-1}^{h}\right)(x, v) f_{k-2+N-i}^{h}(x, v) \mathrm{d} x \mathrm{~d} v \\
\leq & \sum_{k=1}^{T / h} \sum_{i=0}^{N-1} h^{2}\left(\int_{\Omega \times \mathbb{R}^{d}}\left|v \cdot \nabla_{x} G^{\prime}\left(f_{k-1}^{h}\right)(x, v)\right|^{p} \mathrm{~d} x \mathrm{~d} v\right)^{1 / p}\left\|f_{0}\right\|_{L^{\infty}} \\
& +\sum_{k=1}^{T / h} \sum_{i=0}^{N-1} h^{2}\left(\int_{\Omega \times \mathbb{R}^{d}}\left|v \cdot \nabla_{x} G^{\prime}\left(f_{k-1+N}^{h}\right)(x, v)\right|^{p} \mathrm{~d} x \mathrm{~d} v\right)^{1 / p}\left\|f_{0}\right\|_{L^{\infty}} .
\end{aligned}
$$

Next, we use the fact that $v \cdot \nabla_{x} G^{\prime}\left(f_{k}^{h}\right)=G^{\prime \prime}\left(f_{k}^{h}\right) v$. $\nabla_{x} f_{k}^{h}$ and the boundedness in $L^{p}\left(\Omega \times \mathbb{R}^{d}\right)$, of $\left(v \cdot \nabla_{x} f_{k}^{h}\right)_{h, k}$ (see Lemma 8), to get

$$
\begin{aligned}
D \leq 2 & \left\|f_{0}\right\|_{L^{\infty}}\left(\Omega \times \mathbb{R}^{d}\right) \\
& N \frac{T}{h} \sup _{s \in[N, M]} G^{\prime \prime}(s) K_{1}(\Omega) h^{2} \\
& =2\left\|f_{0}\right\|_{L^{\infty}\left(\Omega \times \mathbb{R}^{d}\right)} \sup _{s \in[N, M]} G^{\prime \prime}(s) K_{1}(\Omega) T \tau .
\end{aligned}
$$

Thus,

$$
A=C+D \leq K_{2}\left(A_{1}, \Omega, T, N, M, f_{0}, G^{\prime}, q, p\right) \tau,
$$

where $\quad K_{2}\left(A_{1}, \Omega, T, N, M, f_{0}, G^{\prime}, q, p\right) \quad:=2\left\|f_{0}\right\|_{L^{\infty}\left(\Omega \times \mathbb{R}^{d}\right)}$ $\sup _{s \in[N, M]} G^{\prime \prime}(s) \quad K_{1}(\Omega) T+\left[G^{\prime}(M)-G^{\prime}(N)\right]^{1 / q}$ $\left(M_{0}(T, \Omega)\right)^{1 / p}$.

Let us process now to the following estimate.

Lemma 10. Assume that $f_{0}$ satisfies $\left(H_{f_{0}}\right)$. Then, for every $0<T<\infty$ fixed and for all $\tau>0$ small, we have 


$$
\begin{aligned}
& \int_{[0, T] \times \Omega \times \mathbb{R}^{d}}\left|f^{h}(t+\tau, x, v)-f^{h}(t, x, v)\right|^{p} \mathrm{~d} t \mathrm{~d} x \mathrm{~d} v \\
& \quad \leq C\left(A_{1}, \Omega, T, N, M, f_{0}, G^{\prime}, q, p\right) \tau,
\end{aligned}
$$

where $C\left(A_{1}, \Omega, T, N, M, f_{0}, G^{\prime}, q, p\right)$ is positive constant.

Proof. Since $G \in C^{2}$, we use Taylor's formula to have that

$$
\begin{aligned}
& G^{\prime}\left(f^{h}(t+\tau, x, v)-G^{\prime}\left(f^{h}(t, x, v)\right)\right. \\
& =G^{\prime \prime}\left(f_{\theta}^{h}\right)\left(f^{h}(t+\tau, x, v)-f^{h}(t, x, v)\right),
\end{aligned}
$$

where $\theta \in] 0,1\left[\right.$ and $f_{\theta}^{h}:=\theta f^{h}(t)+(1-\theta) f^{h}(t+\tau)$.

Since $0<N \leq f_{0} \leq M$, we have $0<N \leq f^{h} \leq M$ (see Lemma 2). Thus, using (171) and the fact that $G^{\prime \prime}$ is continuous and nonnegative, we have

$$
\begin{aligned}
& \int_{[0, T] \times \Omega \times \mathbb{R}^{d}}\left[G^{\prime}\left(f^{h}(t+\tau)\right)-G^{\prime}\left(\left(f^{h}\right)\right)\right]\left[f^{h}(t+\tau)-f^{h}(t)\right] \mathrm{d} t \mathrm{~d} x \mathrm{~d} v \\
& \geq \inf _{t \in[N, M]} G^{\prime \prime}(t) \int_{[0, T] \times \Omega \times \mathbb{R}^{d}}\left|f^{h}(t+\tau, x, v)-f^{h}(t, x, v)\right|^{2} \mathrm{~d} t \mathrm{~d} x \mathrm{~d} v .
\end{aligned}
$$

Define $\beta=\inf _{t \in[N, M]} G^{\prime \prime}(t)>0$. We use Lemma 9 and obtain

$$
\begin{aligned}
& \int_{[0, T] \times \Omega \times \mathbb{R}^{d}}\left|f^{h}(t+\tau, x, v)-f^{h}(t, x, v)\right|^{2} \mathrm{~d} t \mathrm{~d} x \mathrm{~d} v \\
& \leq \frac{1}{\beta} K_{2}\left(A_{1}, \Omega, T, N, M, f_{0}, G^{\prime}, q, p\right) \tau .
\end{aligned}
$$

Using $N \leq f^{h} \leq M$, we have

$$
\begin{gathered}
\int_{[0, T] \times \Omega \times \mathbb{R}^{d}}\left|f^{h}(t+\tau, x, v)-f^{h}(t, x, v)\right|^{p} \mathrm{~d} t \mathrm{~d} x \mathrm{~d} v \\
\leq \frac{1}{\beta} C\left(A_{1}, \Omega, T, N, M, f_{0}, G^{\prime}, q, p\right) \tau,
\end{gathered}
$$

where $C\left(\Omega, T, N, M, f_{0}, G^{\prime}, q, p\right)$ is positive constant.

We will use Lemmas 7,8 , and 10 , to prove that the sequence $\left(f^{h}\right)_{h}$ is precompact in $L^{p}\left([0, T] \times \Omega \times \mathbb{R}^{d}\right)$.

Lemma 11. Assume that $f_{0}, c$, and $G$ satisfy, respectively, $\left(H_{f_{0}}\right),\left(H_{c}\right)$, and $\left(H_{G}\right)$. Then, $\left(f^{h}\right)_{h}$ is precompact in $L^{p}\left([0, T] \times \Omega \times B_{R}\right)$, where $B_{R}$ is the open ball centered at origin of radius $R$.

Proof. Define $K=[0, T] \times \Omega \times B_{R}$. Then, $K=\cup_{\delta>0} K^{\delta}$, with $K^{\delta}=\left\{y \in K, \operatorname{dist}\left(y, K^{c}\right)>\delta\right\} . K^{\delta} \subset K, \overline{K^{\delta}} \subset K$, and $\overline{K^{\delta}}$ is compact and $\operatorname{dist}\left(K^{\delta}, K^{c}\right)>\delta / 2$. So, there exists $\delta_{1}, \delta_{2}, \ldots, \delta_{l}>0$ such that $\overline{K^{\delta}} \subset \cup_{i=1}^{l} K^{\delta_{i}}$.

Define $\delta_{0}=\min _{i=1,2, \ldots, d} \delta_{i}$ and we have $K^{\delta_{0}} \Subset K$ and $\operatorname{dist}\left(K^{\delta_{0}}, K^{c}\right)>\delta_{0} / 2$.

Let $\tau>0, \eta_{1}, \eta_{2} \in \mathbb{R}^{d}$, such that $0<\tau<\delta_{0, \varepsilon},\left|\eta_{1}\right|<\delta_{0, \varepsilon}$, and $\left|\eta_{2}\right|<\delta_{0, \varepsilon}$, where $\delta_{0, \varepsilon}=\min \left\{\delta_{0} / 2, \varepsilon\right\}$.

We use Lemmas 7,8 , and 10 to have

$$
\begin{aligned}
& \int_{K^{\delta}}\left|f^{h}(t+\tau, x, v)-f^{h}(t, x, v)\right|^{p} \mathrm{~d} t \mathrm{~d} x \mathrm{~d} v<c_{1} \varepsilon, \\
& \int_{K^{\delta}}\left|f^{h}\left(t, x+\eta_{1}, v\right)-f^{h}(t, x, v)\right|^{p} \mathrm{~d} t \mathrm{~d} x \mathrm{~d} v<\varepsilon^{p} c_{2},
\end{aligned}
$$

$\int_{K^{\delta}}\left|f^{h}\left(t, x, v+\eta_{2}\right)-f^{h}(t, x, v)\right|^{p} \mathrm{~d} t \mathrm{~d} x \mathrm{~d} v<c_{3} \varepsilon^{p}$.

$c_{1}, c_{2}$, and $c_{3}$ are defined in Lemmas 7,8 , and 10 . So, by using the Riesz-Fréchet-Kolmogorov's theorem, we deduce that the sequence $\left(f^{h}\right)_{h}$ is precompact in $L^{P}\left(K^{\delta}\right)$. Therefore, $\lim _{\delta \longrightarrow 0}\left|K \backslash K^{\delta}\right|=0$, and then for all $\varepsilon>0$, there exists $\delta_{\varepsilon}>0$ such that $\left|K \backslash K^{\delta_{\varepsilon}}\right|<\varepsilon\left\|f_{0}\right\|_{L^{\infty}}$, and since $\left(f^{h}\right)_{h}$ is bounded in $L^{\infty}\left([0, T] \times \Omega \times B_{R}\right)$, we have $\left\|f^{h}\right\|_{L^{p}\left(K \backslash K^{\delta_{\varepsilon}}\right)}^{p^{p}} \leq\left\|f_{0}\right\|_{L^{\infty}}$ $\left|K \backslash K^{\delta_{\varepsilon}}\right|<\varepsilon$.

We use corollary $I V .26$ of [9], to conclude that the sequence $\left(f^{h}\right)_{h}$ is precompact in $L^{p}\left([0, T] \times \Omega \times B_{R}\right)$, which implies that $\left(f^{h}\right)_{h}$ converges strongly to some function $f_{R}$ in $L^{p}\left([0, T] \times \Omega \times B_{R}\right)$.

Now, let us prove that the sequence $\left(f^{h}\right)_{h}$ is precompact in $L^{p}\left([0, T] \times \Omega \times \mathbb{R}^{d}\right)$.

Lemma 12. Assume that $f_{0}, c$, and $G$ satisfy, respectively, $\left(H_{f_{0}}\right),\left(H_{c}\right)$, and $\left(H_{G}\right)$. Then, the sequence $\left(f^{h}\right)_{h}$ is precompact in $L^{p}\left([0, T] \times \Omega \times \mathbb{R}^{d}\right)$.

Proof. Since $f_{0} \in L^{p}\left([0, T] \times \Omega \times \mathbb{R}^{d}\right)$, Lemma 3 implies that $\left(f^{h}\right)_{h}$ is bounded in $L^{p}\left([0, T] \times \Omega \times \mathbb{R}^{d}\right)$. Then, $\left(f^{h}\right)_{h}$ converges weakly to some function $f$ in $L^{p}\left([0, T] \times \Omega \times \mathbb{R}^{d}\right)$ (up to a subsequence).

Let $B_{n}$ be an open ball of radius $n$ centered at origin, with $n \in \mathbb{N}^{*}$. Then, $B_{n} \subset B_{n+1}$ and $\cup_{n \in \mathbb{N}^{*}} B_{n}=\mathbb{R}^{d}$.

Using previous results, the sequence $\left(f^{h}\right)_{h}$ is precompact in $L^{p}\left([0, T] \times \Omega \times B_{n}\right)$. A subsequence $\left(f^{h}\right)_{h}$ converges strongly to some function $f_{n}$ in $L^{p}\left([0, T] \times \Omega \times B_{n}\right)$. Let us denote by $\left(f^{h_{n}}\right)$ the subsequence of $\left(f^{h}\right)_{h}$ which converges strongly to $f_{n}$ in $L^{p}\left([0, T] \times \Omega \times B_{n}\right)$. Fix $\phi$ in $L^{q}\left([0, T] \times \Omega \times \mathbb{R}^{d}\right)$. We have

$$
\begin{aligned}
& \int_{[0, T] \times \Omega \times \mathbb{R}^{d}}\left(f^{h_{n}}-f_{n}\right) \phi \mathrm{d} t \mathrm{~d} x \mathrm{~d} v=\int_{[0, T] \times \Omega \times B_{n}}\left(f^{h_{n}}-f_{n}\right) \phi \mathrm{d} t \mathrm{~d} x \mathrm{~d} v \\
& +\int_{[0, T] \times \Omega \times B_{n}^{c}}\left(f^{h_{n}}-f_{n}\right) \phi \mathrm{d} t \mathrm{~d} x \mathrm{~d} v,
\end{aligned}
$$

with

$$
\left|\int_{[0, T] \times \Omega \times B_{n}^{c}}\left(f^{h_{n}}-f_{n}\right) \phi \mathrm{d} t \mathrm{~d} x \mathrm{~d} v\right| \leq\|\phi\|_{L^{q}\left([0, T] \times \Omega \times \mathbb{R}^{d}\right)} 2 M\left|B_{n}^{c}\right|^{1 / p} .
$$

$\left|B_{n}^{c}\right|$ tends to 0 , when $n$ goes to $\infty$; for $\varepsilon>0$, there exists $N_{\varepsilon}>0$ such that

$$
\left|\int_{[0, T] \times \Omega \times B_{n}^{c}}\left(f^{h_{n}}-f_{n}\right) \phi \mathrm{d} t \mathrm{~d} x \mathrm{~d} v\right| \leq \frac{\varepsilon}{2},
$$

for all $n \geq N_{\varepsilon}$. Since $f^{h_{n}}$ converges strongly to $f_{n}$ in $L^{p}\left([0, T] \times \Omega \times B_{n}\right)$, we deduce that 


$$
\left|\int_{[0, T] \times \Omega \times \mathbb{R}^{d}}\left(f^{h_{n}}-f_{n}\right) \phi \mathrm{d} t \mathrm{~d} x \mathrm{~d} v\right| \leq \varepsilon,
$$

when $h_{n}$ goes to 0 and $n \geq N_{\varepsilon}$. We conclude that $\left(f^{h_{n}}\right)$ converges weakly to $f_{n}$ in $L^{p}\left([0, T] \times \Omega \times \mathbb{R}^{d}\right)$, for $n \geq N_{\varepsilon}$. So $f_{n}=f$ for $n \geq N_{\varepsilon}$.

Now, let prove that $\left\|f^{h_{n}}-f\right\|_{L^{p}\left([0, T] \times \Omega \times \mathbb{R}^{d}\right)}$ tends to 0 as $h_{n} \longrightarrow 0$ and $n \geq N_{\varepsilon}$.

Note that

$$
\begin{aligned}
& \left\|f^{h_{n}}-f\right\|_{L^{p}\left([0, T] \times \Omega \times \mathbb{R}^{d}\right)}^{p} \\
& =\int_{[0, T] \times \Omega \times B_{n}}\left|f^{h_{n}}-f\right|^{p} \mathrm{~d} t \mathrm{~d} x \mathrm{~d} v+\int_{[0, T] \times \Omega \times B_{n}^{c}}\left|f^{h_{n}}-f\right|^{p} \mathrm{~d} t \mathrm{~d} x \mathrm{~d} v \\
& \leq \int_{[0, T] \times \Omega \times B_{n}}\left|f^{h_{n}}-f\right|^{p} \mathrm{~d} t \mathrm{~d} x \mathrm{~d} v+2^{p}\left|f_{0}\right|_{L^{\infty}}^{p} T|\Omega \|| B_{n}^{c} \mid .
\end{aligned}
$$

Let us recall that

$$
\lim _{h_{n} \longrightarrow 0} \int_{[0, T] \times \Omega \times B_{n}}\left|f^{h_{n}}-f\right|^{p} \mathrm{~d} t \mathrm{~d} x \mathrm{~d} v=0, \quad \forall n \geq N_{\varepsilon} .
$$

Since $\left|B_{n}^{c}\right|<(\varepsilon / 2)$, for $n \geq N_{\varepsilon}$, as

$$
\lim _{h_{n} \longrightarrow 0} \int_{[0, T] \times \Omega \times B_{N_{\varepsilon}}}\left|f^{h_{n}}-f\right|^{p} \mathrm{~d} t \mathrm{~d} x \mathrm{~d} v=0,
$$

we can find $n_{\varepsilon} \in \mathbb{N}$ such that for every $n \geq N_{\varepsilon}$, we have

$$
\int_{[0, T] \times \Omega \times B_{N_{\varepsilon}}}\left|f^{h_{n}}-f\right|^{p} \mathrm{~d} t \mathrm{~d} x \mathrm{~d} v<\frac{\varepsilon}{2} .
$$

Then, for all $n \geq N_{\varepsilon}$,

$$
\int_{[0, T] \times \Omega \times \mathbb{R}^{d}}\left|f^{h_{n}}-f\right|^{p} \mathrm{~d} t \mathrm{~d} x \mathrm{~d} v<\varepsilon .
$$

Consequently, $\left(f^{h_{n}}\right)$ converges strongly to $f$ in $L^{p}\left([0, T] \times \Omega \times \mathbb{R}^{d}\right)$.

Using the previous lemma, $\left(f^{h}\right)_{h}$ converges strongly to $f$ in $L^{p}\left([0, T] \times \Omega \times \mathbb{R}^{d}\right)$ (up to a subsequence). $G^{\prime}$ is being continuous, then $\left(G^{\prime}\left(f^{h}\right)\right)_{h}$ converges strongly to $G^{\prime}(f)$ in $L^{p}\left([0, T] \times \Omega \times \mathbb{R}^{d}\right)$. We conclude that the sequence $\nabla_{v} G^{\prime}\left(f^{h}\right)_{h}$ converges to $\nabla_{v} G^{\prime}(f)$ in $\left[C_{c}^{\infty}\left(\mathbb{R} \times \Omega \times \mathbb{R}^{d}\right)\right]^{\prime}$. Let us recall that $\left(\nabla_{v} G^{\prime}\left(f^{h}\right)\right)_{h}$ is bounded in $L^{p}\left([0, T] \times \Omega \times \mathbb{R}^{d}\right)$, so $\left(\nabla_{v} G^{\prime}\left(f^{h}\right)\right)_{h}$ converges weakly to $\nabla_{v} G^{\prime}(f)$ in $L^{p}\left([0, T] \times \Omega \times \mathbb{R}^{d}\right)$.

On the contrary, $\left(\nabla c^{*}\left(\nabla_{v} G^{\prime}\left(f^{h}\right)\right)\right)_{h}$ is bounded in $L^{q}\left([0, T] \times \Omega \times \mathbb{R}^{d}\right)$. Then, a subsequence $\left(\nabla c^{*}\left(\nabla_{v} G^{\prime}\left(f^{h}\right)\right)\right)_{h}$ converges weakly to $\sigma$ in $L^{q}\left([0, T] \times \Omega \times \mathbb{R}^{d}\right)$.

We derive from the proof of Theorem 3.10 in [1] that the sequence $\operatorname{div}_{v}\left(f^{h} \nabla c^{*}\left(\nabla_{v} G^{\prime}\left(f^{h}\right)\right)\right)$ converges weakly to $\operatorname{div}_{v}\left(f \nabla c^{*}\left(\nabla_{v} G^{\prime}(f)\right)\right)$ in $\left[C_{c}^{\infty}\left(\mathbb{R} \times \Omega \times \mathbb{R}^{d}\right)\right]^{\prime}$.

Theorem 2. Assume that $f_{0}, c$, and $G$ satisfy, respectively, $\left(H_{f_{0}}\right),\left(H_{c}\right)$, and $\left(H_{G}\right)$. Let $0<T<\infty$ and $u \in C_{c}^{\infty}(\mathbb{R})$ be such that $u \geq 0$ and $\sup p u \subset[-T, T]$. Then,

$$
\begin{aligned}
& \lim _{h \longrightarrow 0} \int_{[0, T] \times \Omega \times \mathbb{R}^{d}}\left\langle f^{h} \nabla c^{*}\left(\nabla_{v} G^{\prime}\left(f^{h}\right)\right), \nabla_{v} G^{\prime}\left(f^{h}\right)\right\rangle u(t) \mathrm{d} t \mathrm{~d} x \mathrm{~d} v \\
& =\int_{[0, T] \times \Omega \times \mathbb{R}^{d}}\left\langle f \sigma, \nabla_{\nu} G^{\prime}(f)\right\rangle u(t) \mathrm{d} t \mathrm{~d} x \mathrm{~d} v .
\end{aligned}
$$

Moreover, $\left(\operatorname{div}_{v}\left(f^{h} \nabla c^{*}\left(\nabla_{v} G^{\prime}\left(f^{h}\right)\right)\right)\right)_{h}$ converges weakly to $\operatorname{div}_{v}(f \sigma)$ in $\left[C_{c}^{\infty}\left(\mathbb{R} \times \Omega \times \mathbb{R}^{d}\right)\right]^{\prime}$ and $\operatorname{div}_{v}(f \sigma)=$ $\operatorname{div}_{v}\left(f \nabla c^{*}\left(\nabla_{v} G^{\prime}(f)\right)\right)$ in the weak sense.

Proof. Let us recall the following:

(i) $\left(f^{h}\right)_{h} \quad$ converges strongly to $f$ in $L^{p}\left([0, T] \times \Omega \times \mathbb{R}^{d}\right)$

(ii) $\left(\nabla_{v} G^{\prime}\left(f^{h}\right)\right)_{h}$ converges weakly to $\nabla_{v} G^{\prime}(f)$ in $L^{p}\left([0, T] \times \Omega \times \mathbb{R}^{d}\right)$

(iii) $\left(\sigma^{h}=\nabla c^{*}\left(\nabla_{v} G^{\prime}\left(f^{h}\right)\right)\right)_{h}$ converges weakly to $\sigma$ in $L^{q}\left([0, T] \times \Omega \times \mathbb{R}^{d}\right)$

Then, we have

$$
\begin{aligned}
& \int_{[0, T] \times \Omega \times \mathbb{R}^{d}}\left\langle f^{h} \nabla c^{*}\left(\nabla_{v} G^{\prime}\left(f^{h}\right)\right), \nabla_{v} G^{\prime}(f)\right\rangle u(t) \mathrm{d} t \mathrm{~d} x \mathrm{~d} v \\
& =\int_{[0, T] \times \Omega \times \mathbb{R}^{d}}\left(f^{h}-f\right)\left\langle\nabla c^{*}\left(\nabla G^{\prime}\left(f^{h}\right)\right), \nabla_{\nu} G^{\prime}(f)\right\rangle u(t) \mathrm{d} t \mathrm{~d} x \mathrm{~d} v \\
& \quad+\int_{[0, T] \times \Omega \times \mathbb{R}^{d}} f\left\langle\nabla c^{*}\left(\nabla_{\nu} G^{\prime}\left(f^{h}\right)\right), \nabla_{v} G^{\prime}(f)\right\rangle u(t) \mathrm{d} t \mathrm{~d} x \mathrm{~d} v .
\end{aligned}
$$

Since $\quad \nabla_{v}\left(G^{\prime}(f)\right) \in L^{p}\left([0, T] \times \Omega \times \mathbb{R}^{d}\right) \quad$ and $\left(\nabla c^{*}\left(\nabla_{v} G^{\prime}\left(f^{h}\right)\right)\right)_{h}$ is bounded in $L^{q}\left([0, T] \times \Omega \times \mathbb{R}^{d}\right)$, we use (i) and (iii) to have

$$
\begin{aligned}
& \lim _{h \rightarrow 0} \int_{[0, T] \times \Omega \times \mathbb{R}^{d}}\left\langle f^{h} \sigma^{h}, \nabla_{v} G^{\prime}(f)\right\rangle u(t) \mathrm{d} t \mathrm{~d} x \mathrm{~d} v \\
& =\int_{[0, T] \times \Omega \times \mathbb{R}^{d}}\left\langle f \sigma, \nabla_{v} G^{\prime}(f)\right\rangle u(t) \mathrm{d} t \mathrm{~d} x \mathrm{~d} v .
\end{aligned}
$$

Also, we have

$\int_{[0, T] \times \Omega \times \mathbb{R}^{d}}\left\langle f^{h} \nabla c^{*}\left(\nabla_{v} G^{\prime}(f)\right), \nabla_{v} G^{\prime}\left(f^{h}\right)-\nabla_{v} G^{\prime}(f)\right\rangle u(t) \mathrm{d} t \mathrm{~d} x \mathrm{~d} v$

$=\int_{[0, T] \times \Omega \times \mathbb{R}^{d}}\left\langle\left(f^{h}-f\right) \nabla c^{*}\left(\nabla_{v} G^{\prime}(f)\right), \nabla_{v} G^{\prime}\left(f^{h}\right)-\nabla_{v} G^{\prime}(f)\right\rangle u(t) \mathrm{d} t \mathrm{~d} x \mathrm{~d} v$

$+\int_{[0, T] \times \Omega \times \mathbb{R}^{d}}\left\langle f \nabla c^{*}\left(\nabla_{\nu} G^{\prime}(f)\right), \nabla_{v} G^{\prime}\left(f^{h}\right)-\nabla_{v} G^{\prime}(f)\right\rangle u(t) \mathrm{d} t \mathrm{~d} x \mathrm{~d} v$.

Using (i), (ii), and the fact that $\nabla c^{*}\left(\nabla_{v} G^{\prime}(f)\right) \in L^{q}\left([0, T] \times \Omega \times \mathbb{R}^{d}\right)$ and that $\left(\nabla_{v} G^{\prime}\left(f^{h}\right)\right)_{h}$ is bounded in $L^{p}\left([0, T] \times \Omega \times \mathbb{R}^{d}\right)$, we get

$$
\begin{aligned}
& \lim _{h \longrightarrow 0} \int_{[0, T] \times \Omega \times \mathbb{R}^{d}}\left\langle f^{h} \nabla c^{*}\left(\nabla_{v} G^{\prime}(f)\right), \nabla_{v} G^{\prime}\left(f^{h}\right)-\nabla_{v} G^{\prime}(f)\right\rangle \\
& u(t) \mathrm{d} t \mathrm{~d} x \mathrm{~d} v=0 .
\end{aligned}
$$

To conclude with Theorem 2, we need to establish the following limit, whose proof is derived from the three following lemmas: 
$\lim _{h \longrightarrow 0} \int_{\left[0, \infty\left[\times \Omega \times \mathbb{R}^{d}\right.\right.}\left\langle f^{h} \nabla c^{*}\left(\nabla_{v} G^{\prime}\left(f^{h}\right)\right), \nabla_{v} G^{\prime}\left(f^{h}\right)\right\rangle u(t) \mathrm{d} t \mathrm{~d} x \mathrm{~d} v$

$=\int_{\left[0, \infty\left[\times \Omega \times \mathbb{R}^{d}\right.\right.}\left\langle f \sigma, \nabla_{v} G^{\prime}(f)\right\rangle u(t) \mathrm{d} t \mathrm{~d} x \mathrm{~d} v$.

\section{Lemma 13}

$$
\begin{aligned}
& \liminf _{h \longrightarrow 0} \int_{[0, T] \times \Omega \times \mathbb{R}^{d}}\left\langle f^{h} \sigma^{h}, \nabla_{\nu} G^{\prime}\left(f^{h}\right)\right\rangle u(t) \mathrm{d} t \mathrm{~d} x \mathrm{~d} v \\
& \geq \int_{[0, T] \times \Omega \times \mathbb{R}^{d}}\left\langle f \sigma, \nabla_{v} G^{\prime}(f)\right\rangle u(t) \mathrm{d} t \mathrm{~d} x \mathrm{~d} v .
\end{aligned}
$$

Proof. Since $c^{*}$ is convex and $f^{h} \geq 0, u \geq 0$, then

$$
\begin{aligned}
f^{h} & \left\langle\nabla c^{*}\left(\nabla_{\nu} G^{\prime}\left(f^{h}\right)\right)-\nabla c^{*}\left(\nabla_{\nu} G^{\prime}(f)\right), \nabla_{\nu} G^{\prime}\left(f^{h}\right)\right. \\
& \left.-\nabla_{\nu} G^{\prime}(f)\right\rangle u(t) \geq 0 .
\end{aligned}
$$

So,

$$
\begin{aligned}
& \int_{[0, T] \times \Omega \times \mathbb{R}^{d}}\left\langle f^{h} \nabla c^{*}\left(\nabla_{v} G^{\prime}\left(f^{h}\right), \nabla_{v} G^{\prime}\left(f^{h}\right)\right\rangle u(t) \mathrm{d} t \mathrm{~d} x \mathrm{~d} v\right. \\
& \geq \int_{[0, T] \times \Omega \times \mathbb{R}^{d}}\left\langle f^{h} \nabla c^{*}\left(\nabla_{v} G^{\prime}\left(f^{h}\right), \nabla_{v} G^{\prime}(f)\right\rangle u(t) \mathrm{d} t \mathrm{~d} x \mathrm{~d} v\right. \\
& \quad+\int_{[0, T] \times \Omega \times \mathbb{R}^{d}}\left\langle f^{h} \nabla c^{*}\left(\nabla_{v} G^{\prime}(f)\right), \nabla_{v} G^{\prime}\left(f^{h}\right)-\nabla_{\nu} G^{\prime}(f)\right\rangle \\
& \quad u(t) \mathrm{d} t \mathrm{~d} x \mathrm{~d} v .
\end{aligned}
$$

Passing to the limit, we obtain

$$
\begin{aligned}
& \liminf _{h \longrightarrow 0} \int_{[0, T] \times \Omega \times \mathbb{R}^{d}}\left\langle f^{h} \nabla c^{*}\left(\nabla_{\nu} G^{\prime}\left(f^{h}\right), \nabla_{\nu} G^{\prime}\left(f^{h}\right)\right\rangle u(t) \mathrm{d} t \mathrm{~d} x \mathrm{~d} v\right. \\
& \geq \liminf _{h \longrightarrow 0} \int_{[0, T] \times \Omega \times \mathbb{R}^{d}}\left\langle f^{h} \nabla c^{*}\left(\nabla_{\nu} G^{\prime}\left(f^{h}\right), \nabla_{\nu} G^{\prime}(f)\right\rangle u(t) \mathrm{d} t \mathrm{~d} x \mathrm{~d} v\right. \\
& \quad+\liminf _{h \longrightarrow 0} \int_{[0, T] \times \Omega \times \mathbb{R}^{d}}\left\langle f^{h} \nabla c^{*}\left(\nabla_{v} G^{\prime}(f)\right), \nabla_{v} G^{\prime}\left(f^{h}\right)\right. \\
& \left.\quad-\nabla_{\nu} G^{\prime}(f)\right\rangle u(t) \mathrm{d} t \mathrm{~d} x \mathrm{~d} v .
\end{aligned}
$$

Taking into account (187) and (189) in (194) enables us to conclude the proof of Lemma 13.

\section{Lemma 14}

$$
\begin{aligned}
& \limsup _{h \longrightarrow 0} \int_{[0, T] \times \Omega \times \mathbb{R}^{d}}\left\langle f^{h} \sigma^{h}, \nabla_{v} G^{\prime}\left(f^{h}\right)\right\rangle u(t) \mathrm{d} t \mathrm{~d} x \mathrm{~d} v \\
& \leq \int_{\Omega \times \mathbb{R}^{d}}\left[f_{0} G^{\prime}\left(f_{0}\right)-G^{*}\left(G^{\prime}\left(f_{0}\right)\right)\right] u(0) \mathrm{d} x \mathrm{~d} v \\
& \quad+\int_{[0, T] \times \Omega \times \mathbb{R}^{d}}\left[f G^{\prime}(f)-G^{*}\left(G^{\prime}(f)\right)\right] u \prime(t) \mathrm{d} t \mathrm{~d} x \mathrm{~d} v .
\end{aligned}
$$

Proof. Using the proof of Lemma 6, we have

$$
\begin{aligned}
& \int_{\Omega \times \mathbb{R}^{d}} G\left(f_{k-1}^{h}\right) \mathrm{d} x \mathrm{~d} v-\int_{\Omega \times \mathbb{R}^{d}} G\left(f_{k}^{h}\right) \mathrm{d} x \mathrm{~d} v \\
& \geq \int_{\Omega \times \mathbb{R}^{d}}\left\langle T_{k}(v)-v, \nabla_{v} G^{\prime}\left(f_{k}^{h}\right)\right\rangle f_{k}^{h} \mathrm{~d} x \mathrm{~d} v,
\end{aligned}
$$

where

$$
T_{k}(v)=v+h \nabla c^{*}\left(\nabla_{v} G^{\prime}\left(f_{k}^{h}\right)\right),
$$

is a $c$-optimal map that pushes $F_{k}^{x h}$ forward $G_{k}^{x h}$. Thus,

$$
\begin{aligned}
& \int_{\Omega \times \mathbb{R}^{d}} \frac{G\left(f_{k}^{h}\right)-G\left(f_{k-1}^{h}\right)}{h} \mathrm{~d} x \mathrm{~d} v \\
& \leq-\int_{\Omega \times \mathbb{R}^{d}}\left\langle\nabla c^{*}\left(\nabla_{v} G^{\prime}\left(f_{k}^{h}\right)\right), \nabla_{v} G^{\prime}\left(f_{k}^{h}\right)\right\rangle f_{k}^{h} \mathrm{~d} x \mathrm{~d} v .
\end{aligned}
$$

Since $u \geq 0$, then

$\sum_{k=1}^{T / h} \int_{t_{k-1}}^{t_{k}} \int_{\Omega \times \mathbb{R}^{d}} \frac{G\left(f_{k}^{h}\right)-G\left(f_{k-1}^{h}\right)}{h} u(t) \mathrm{d} x \mathrm{~d} v$

$\leq-\int_{[0, T] \times \Omega \times \mathbb{R}^{d}}\left\langle\nabla c^{*}\left(\nabla_{\nu} G^{\prime}\left(f^{h}\right)\right), \nabla_{\nu} G^{\prime}\left(f^{h}\right)\right\rangle f^{h} u(t) \mathrm{d} t \mathrm{~d} x \mathrm{~d} v$.

Also,

$$
\begin{gathered}
\sum_{k=1}^{T / h} \int_{t_{k-1}}^{t_{k}} \int_{\Omega \times \mathbb{R}^{d}} \frac{G\left(f_{k}^{h}\right)-G\left(f_{k-1}^{h}\right)}{h} u(t) \mathrm{d} x \mathrm{~d} v \\
=-\int_{[0, T] \times \Omega \times \mathbb{R}^{d}} G\left(f^{h}\right) \partial_{t}^{h} u(t) \mathrm{d} t \mathrm{~d} x \mathrm{~d} v \\
-\frac{1}{h} \int_{0}^{h} \int_{\Omega \times \mathbb{R}^{d}} G\left(f_{0}\right) u(t) \mathrm{d} t \mathrm{~d} x \mathrm{~d} v,
\end{gathered}
$$

where $\partial_{t}^{h} u(t)=(u(t+h)-u(t) / h)$.

Let us prove that

$$
\begin{gathered}
\lim _{h \rightarrow 0} \int_{[0, T] \times \Omega \times \mathbb{R}^{d}} G\left(f^{h}\right) \partial_{t}^{h} u(t) \mathrm{d} t \mathrm{~d} x \mathrm{~d} v \\
\quad=\int_{[0, T] \times \Omega \times \mathbb{R}^{d}} G(f) u \prime(t) \mathrm{d} t \mathrm{~d} x \mathrm{~d} v .
\end{gathered}
$$

Indeed,

$$
\begin{aligned}
& \left|\int_{[0, T] \times \Omega \times \mathbb{R}^{d}} G\left(f^{h}\right) \partial_{t}^{h} u(t)-G(f) u \prime(t) \mathrm{d} t \mathrm{~d} x \mathrm{~d} v\right| \\
& \leq \int_{[0, T] \times \Omega \times \mathbb{R}^{d}}\left|G\left(f^{h}\right)-G(f)\right||u \prime(t)| \mathrm{d} t \mathrm{~d} x \mathrm{~d} v \\
& \quad+\int_{[0, T] \times \Omega \times \mathbb{R}^{d}}\left|\partial_{t}^{h} u(t)-u \prime(t)\right|\left|G\left(f^{h}\right)\right| \mathrm{d} t \mathrm{~d} x \mathrm{~d} v .
\end{aligned}
$$

Using (i), the continuity of $G$, the boundedness of $\left(f^{h}\right)_{h}$ in $L^{\infty}\left(\left[0, \infty\left[\times \Omega \times \mathbb{R}^{d}\right)\right.\right.$, and the fact that $\left(\partial_{t}^{h} u\right)_{h}$ converges uniformly to $u$ ' on the compact $[0, T]$, we obtain 


$$
\begin{gathered}
\lim _{h \rightarrow 0} \int_{[0, T] \times \Omega \times \mathbb{R}^{d}} G\left(f^{h}\right) \partial_{t}^{h} u(t) \mathrm{d} t \mathrm{~d} x \mathrm{~d} v \\
\quad=\int_{[0, T] \times \Omega \times \mathbb{R}^{d}} G(f) u \prime(t) \mathrm{d} t \mathrm{~d} x \mathrm{~d} v .
\end{gathered}
$$

Next, we combine (199), (200), and (203) to get

$\int_{[0, T] \times \Omega \times \mathbb{R}^{d}} G(f) u \prime(t) \mathrm{d} t \mathrm{~d} x \mathrm{~d} v+\int_{\Omega \times \mathbb{R}^{d}} G\left(f_{0}\right) u(0) \mathrm{d} x \mathrm{~d} v \geq$

$\limsup _{h \longrightarrow 0} \int_{[0, T] \times \Omega \times \mathbb{R}^{d}} f^{h}\left\langle\nabla c^{*}\left(\nabla_{v} G^{\prime}\left(f^{h}\right)\right), \nabla_{v} G^{\prime}\left(f^{h}\right)\right\rangle u(t) \mathrm{d} t \mathrm{~d} x \mathrm{~d} v$.

Since $G \in C^{1}$ is strictly convex, $G^{*}\left(G^{\prime}(s)\right)=s G^{\prime}(s)-$ $G(s), \forall s>0$. Consequently,

replacing

$G(f)=f G^{\prime}(f)-G^{*}\left(G^{\prime}(f)\right)$

and

$G\left(f_{0}\right)=f_{0} G^{\prime}\left(f_{0}\right)-G^{*}\left(G^{\prime}\left(f_{0}\right)\right)$ in (5), we obtain Lemma 14.

\section{Lemma 15}

$$
\begin{aligned}
& \int_{\Omega \times \mathbb{R}^{d}}\left[f_{0} G^{\prime}\left(f_{0}\right)-G^{*}\left(G^{\prime}\left(f_{0}\right)\right)\right] u(0) \mathrm{d} x \mathrm{~d} v \\
& +\int_{[0, T] \times \Omega \times \mathbb{R}^{d}}\left[f G^{\prime}(f)-G^{*}\left(G^{\prime}(f)\right)\right] u \prime(t) \mathrm{d} t \mathrm{~d} x \mathrm{~d} v \\
& \leq \int_{[0, T] \times \Omega \times \mathbb{R}^{d}}\left\langle f \sigma, \nabla_{v} G^{\prime}(f)\right\rangle u(t) \mathrm{d} t \mathrm{~d} x \mathrm{~d} v .
\end{aligned}
$$

Proof. Let us write $\psi(t, x, v)=G^{\prime}(f) u(t)$ for $(t,, x, v) \in[0, T] \times \Omega \times \mathbb{R}^{d}$. we have $\psi(t, .,.) \in W^{1, p}\left(\Omega \times B_{R}\right)$.

Approximating $\psi(t, .,$.$) by C_{c}^{\infty}\left(\Omega \times \mathbb{R}^{d}\right)$ functions and using (17), we have

$$
\begin{aligned}
& \frac{A_{k}^{h}[\psi]}{h}=\int_{\Omega \times B_{R}} \frac{f_{k}^{h}-g_{k}^{h}}{h} \psi\left(t, x+\left(t-t_{k}\right) v, v\right) \mathrm{d} x \mathrm{~d} v \\
& \quad+\int_{\Omega \times B_{R}} f_{k}^{h}\left\langle\nabla c^{*}\left(\nabla G^{\prime}\left(f_{k}^{h}\right)\right), \nabla_{v} \psi\left(t, x+\left(t-t_{k}\right) v, v\right)\right\rangle \mathrm{d} x \mathrm{~d} v,
\end{aligned}
$$

where $A_{k}^{h}[\psi]$ is defined in (17) and tends to 0 as $h \longrightarrow 0$. By integrating (206) over $[0, T]$, one obtains

$$
\begin{aligned}
& \sum_{i=1}^{T / h} \int_{t_{i}}^{t_{i+1}} \frac{A_{i}^{h}[\psi]}{h}=\sum_{i=1}^{T / h} \int_{t_{i}}^{t_{i+1}} \int_{\Omega \times B_{R}} \frac{f_{i}^{h}-g_{i}^{h}}{h} \psi\left(t, x+\left(t-t_{i}\right) v, v\right) \mathrm{d} t \mathrm{~d} x \mathrm{~d} v \\
& \quad+\sum_{i=1}^{T / h} \int_{t_{i}}^{t_{i+1}} \int_{\Omega \times B_{R}} f_{i}^{h}\left\langle\nabla c^{*}\left(\nabla_{v} G^{\prime}\left(f_{i}^{h}\right), \nabla_{v} \psi\left(t, x+\left(t-t_{i}\right) v, v\right)\right\rangle \mathrm{d} t \mathrm{~d} x \mathrm{~d} v\right.
\end{aligned}
$$

Next, from the definition of $f^{h}$, we derive that

$$
\begin{aligned}
& \sum_{k=1}^{T / h} \int_{t_{k}}^{t_{k+1}} \int_{\Omega \times B_{R}} \frac{f_{k}^{h}-g_{k}^{h}}{h} \psi\left(t, x+\left(t-t_{k}\right) v, v\right) \mathrm{d} t \mathrm{~d} x \mathrm{~d} v \\
& =-\int_{[0, T] \times \Omega \times B_{R}} f^{h}\left[\frac{\psi(t+h, x, v)-\psi(t, x, v)}{h}\right] \mathrm{d} t \mathrm{~d} x \mathrm{~d} v \\
& \quad-\frac{1}{h} \int_{0}^{h} \int_{\Omega \times B_{R}} f^{h} \psi(t, x, v) \mathrm{d} t \mathrm{~d} x \mathrm{~d} v,
\end{aligned}
$$

$\sum_{i=1}^{T / h} \int_{t_{i}}^{t_{i+1}} \int_{\Omega \times B_{R}} f_{i}^{h}\left\langle\nabla c^{*}\left(\nabla_{v} G^{\prime}\left(f_{i}^{h}\right), \nabla_{v} \psi\left(t, x+\left(t-t_{i}\right) v, v\right)\right\rangle \mathrm{d} t \mathrm{~d} x \mathrm{~d} v\right.$

$=\int_{[0, T] \times \Omega \times B_{R}} f^{h}\left\langle\nabla c^{*}\left(\nabla_{v} G^{\prime}\left(f^{h}\right), \nabla_{v} \psi(t, x, v)\right\rangle \mathrm{d} t \mathrm{~d} x \mathrm{~d} v\right.$

$-\int_{0}^{h} \int_{\Omega \times B_{R}} f_{0}\left\langle\nabla c^{*}\left(\nabla_{v} G^{\prime}\left(f_{0}\right), \nabla_{v} \psi(t, x, v)\right\rangle \mathrm{d} t \mathrm{~d} x \mathrm{~d} v\right.$.

We combine (208) and (209) to obtain

$$
\begin{aligned}
& B^{h}[\psi]=-\int_{[0, T] \times \Omega \times B_{R}} f^{h}\left[\frac{G^{\prime}(f(t+h)) u(t+h)-G^{\prime}(f(t)) u(t)}{h}\right] \mathrm{d} t \mathrm{~d} x \mathrm{~d} v \\
& -\frac{1}{h} \int_{0}^{h} \int_{\Omega \times B_{R}} f_{0} G^{\prime}(f) u(t) \mathrm{d} t \mathrm{~d} x \mathrm{~d} v \\
& +\int_{[0, T] \times \Omega \times B_{R}} f^{h}\left\langle<\nabla c^{*}\left(\nabla_{v} G^{\prime}\left(f^{h}\right), \nabla_{v} \psi\right\rangle \mathrm{d} t \mathrm{~d} x \mathrm{~d} v\right. \\
& -\int_{0}^{h} \int_{\Omega \times B_{R}} f_{0}\left\langle\nabla c ^ { * } \left(\nabla_{v} G^{\prime}\left(f_{0}, \nabla_{v} \psi\right\rangle \mathrm{d} t \mathrm{~d} x \mathrm{~d} v,\right.\right.
\end{aligned}
$$

where

$$
B^{h}[\psi]=\sum_{i=1}^{T / h} \int_{t_{i}}^{t_{i+1}} \frac{A_{i}^{h}[\psi]}{h} \mathrm{~d} t .
$$

Let us show that $B^{h}[\psi]$ tends to 0 as $h \longrightarrow 0$. Assume that $\psi \in C_{c}^{2}\left(\mathbb{R} \times \Omega \times \mathbb{R}^{d}\right)$.

(i) If $q \geq 2$, we use (116) and we have

$$
\begin{aligned}
& \left|A_{k}^{h}[\psi]\right| \leq \frac{h}{2}\left\|\nabla_{v}^{2} \psi\right\|_{L^{\infty}}\left[h \int_{\Omega \times \mathbb{R}^{d}}\left\|\nabla_{v}\left(G^{\prime}\left(f_{k}^{h}\right)\right)\right\|^{p} f_{k}^{h} \mathrm{~d} x \mathrm{~d} v+h\right] \\
& +\frac{h^{2}}{2}\left\|\nabla_{x}^{2} \psi\right\|_{L^{\infty}\left(\Omega \times \mathbb{R}^{d}\right)}\left\|f_{0}\right\|_{L^{\infty}\left(\Omega \times \mathbb{R}^{d}\right)} \int_{\Omega \times K_{\psi}}|v|^{2} \mathrm{~d} x \mathrm{~d} v .
\end{aligned}
$$

Recalling the fact that $c(x) \geq A_{1}|x|^{q}$, we have

$h \int_{\Omega \times \mathbb{R}^{d}}\left|\nabla_{v}\left(G^{\prime}\left(f_{k}^{h}\right)\right)\right|^{p} f_{k}^{h} \mathrm{~d} x \mathrm{~d} v \geq \frac{h}{A_{1}} \int_{\Omega} \rho_{k}^{h}(x) W_{c}^{h}\left(F_{k}^{x h}, G_{k}^{x h}\right) \mathrm{d} x$. 
From the statements in Section 2.1, we get

$\int_{\Omega} \rho_{k}^{h}(x) W_{c}^{h}\left(F_{k}^{x h}, G_{k}^{x h}\right) \mathrm{d} x \leq \frac{1}{A_{1}}\left[\int_{\Omega \times \mathbb{R}^{d}} G\left(f_{k-1}^{h}\right)-\int_{\Omega \times \mathbb{R}^{d}} G\left(f_{k}^{h}\right)\right] \mathrm{d} x \mathrm{~d} v$,

and accordingly,

$\left|A_{k}^{h}[\psi]\right| \leq \frac{h}{2 A_{1}}\left\|\nabla_{v}^{2} \psi\right\|_{L^{\infty}}\left[\int_{\Omega \times \mathbb{R}^{d}} G\left(f_{k-1}^{h}\right) \mathrm{d} x \mathrm{~d} v-\int_{\Omega \times \mathbb{R}^{d}} G\left(f_{k}^{h}\right) \mathrm{d} x \mathrm{~d} v+h\right]$ $+\frac{h^{2}}{2}\left\|\nabla_{x}^{2} \psi\right\|_{L^{\infty}\left(\Omega \times \mathbb{R}^{d}\right)}\left\|f_{0}\right\|_{L^{\infty}\left(\Omega \times \mathbb{R}^{d}\right)} \int_{\Omega \times K_{\psi}}|v|^{2} \mathrm{~d} x \mathrm{~d} v$.

So

$\left|B^{h}[\psi]\right| \leq \frac{1}{2 A_{1}}\left\|\nabla_{v}^{2} \psi\right\|_{L^{\infty}}\left[\sum_{k=1}^{T / h} \int_{t_{k}}^{t_{k+1}}\left(\int_{\Omega \times \mathbb{R}^{d}} G\left(f_{k-1}^{h}\right) \mathrm{d} x \mathrm{~d} v\right.\right.$

$$
\left.\left.-\int_{\Omega \times \mathbb{R}^{d}} G\left(f_{k}^{h}\right) \mathrm{d} x \mathrm{~d} v\right)+h T\right]
$$

$+\frac{h}{2} T\left\|\nabla_{x}^{2} \psi\right\|_{L^{\infty}\left(\Omega \times \mathbb{R}^{d}\right)}\left\|f_{0}\right\|_{L^{\infty}\left(\Omega \times \mathbb{R}^{d}\right)} \int_{\Omega \times K_{\psi}}|v|^{2} \mathrm{~d} x \mathrm{~d} v$,

and then,

$$
\begin{aligned}
& \left|B^{h}[\psi]\right| \leq \frac{1}{2}\left|\nabla_{v}^{2} \psi\right|_{L^{\infty}}\left[h \left(\int_{\Omega \times \mathbb{R}^{d}} G\left(f_{0}\right) \mathrm{d} x \mathrm{~d} v\right.\right. \\
& \left.\left.\quad-\int_{\Omega \times \mathbb{R}^{d}} G\left(f_{T / h}^{h}\right) \mathrm{d} x \mathrm{~d} v\right)+h T\right] \\
& +\frac{h}{2} T\left\|\nabla_{x}^{2} \psi\right\|_{L^{\infty}\left(\Omega \times \mathbb{R}^{d}\right)}\left\|f_{0}\right\|_{L^{\infty}\left(\Omega \times \mathbb{R}^{d}\right)} \int_{\Omega \times K_{\psi}}|v|^{2} \mathrm{~d} x \mathrm{~d} v .
\end{aligned}
$$

From the proof of Lemma 6, we have

$\int_{\Omega \times \mathbb{R}^{d}} G\left(f_{0}\right) \mathrm{d} x \mathrm{~d} v-\int_{\Omega \times \mathbb{R}^{d}} G\left(f_{T / h}^{h}\right) \mathrm{d} x \mathrm{~d} v \leq G^{\prime}(M)-G^{\prime}(N)$.

Then, we obtain

$$
\begin{aligned}
& \left|B^{h}[\psi]\right| \leq \frac{1}{2}\left\|\nabla_{\nu}^{2} \psi\right\|_{L^{\infty}}\left[h\left(G^{\prime}(M)-G^{\prime}(N)\right)+h T\right] \\
& +\frac{h}{2} T\left\|\nabla_{x}^{2} \psi\right\|_{L^{\infty}\left(\Omega \times \mathbb{R}^{d}\right)}\left\|f_{0}\right\|_{L^{\infty}\left(\Omega \times \mathbb{R}^{d}\right)} \int_{\Omega \times K_{\psi}}|v|^{2} \mathrm{~d} x \mathrm{~d} v .
\end{aligned}
$$

So, $B^{h}[\psi]$ tends to 0 as $h \longrightarrow 0$.

(ii) If $q<2$, we have

$\int_{\Omega \times \mathbb{R}^{d}}\left(h\left|\nabla_{\nu} G^{\prime}\left(f_{k}^{h}\right)\right|^{p}\right)^{2 / q} f_{k}^{h} \mathrm{~d} x \mathrm{~d} v \leq \int_{\Omega \times \mathbb{R}^{d}} h\left|\nabla_{v} G^{\prime}\left(f_{k}^{h}\right)\right|^{p} f_{k}^{h} \mathrm{~d} x \mathrm{~d} v$,

and then,

$$
\begin{aligned}
& \left|A_{k}^{h}[\psi]\right| \leq 2 \frac{h^{2-(2 / q)}}{2}\left\|\nabla_{v}^{2} \psi\right\|_{L^{\infty}} \int_{\Omega \times \mathbb{R}^{d}} h\left|\nabla_{v} G^{\prime}\left(f_{k}^{h}\right)\right|^{p} f_{k}^{h} \mathrm{~d} x \mathrm{~d} v \\
& +\frac{h^{2}}{2}\left\|\nabla_{x}^{2} \psi\right\|_{L^{\infty}}\left\|f_{0}\right\|_{L^{\infty}} \int_{\Omega \times K_{\psi}}|v|^{2} \mathrm{~d} x \mathrm{~d} v .
\end{aligned}
$$

From the statements in Section 2.1, we obtain

$$
\begin{aligned}
& \left|A_{k}^{h}[\psi]\right| \leq \frac{h^{2-2 / q}}{2}\left\|\nabla_{v}^{2} \psi\right\|_{L^{\infty}}\left[\int_{\Omega \times \mathbb{R}^{d}} G\left(f_{k-1}^{h}\right) \mathrm{d} x \mathrm{~d} v\right. \\
& \left.-\int_{\Omega \times \mathbb{R}^{d}} G\left(f_{k}^{h}\right) \mathrm{d} x \mathrm{~d} v\right] \\
& +\frac{h^{2}}{2}\left\|\nabla_{x}^{2} \psi\right\|_{L^{\infty}}\left\|f_{0}\right\|_{L^{\infty}} \int_{\Omega \times K_{\psi}}|v|^{2} \mathrm{~d} x \mathrm{~d} v,
\end{aligned}
$$

and then,

$$
\begin{aligned}
& \left|B^{h}[\psi]\right| \leq \frac{h^{2-(2 / q)}}{2}\left\|\nabla_{v}^{2} \psi\right\|_{L^{\infty}}\left[G^{\prime}(M)-G^{\prime}(N)\right] \\
& +\frac{h}{2} T\left\|\nabla_{x}^{2} \psi\right\|_{L^{\infty}}\left\|f_{0}\right\|_{L^{\infty}} \int_{\Omega \times K_{\psi}}|v|^{2} \mathrm{~d} x \mathrm{~d} v .
\end{aligned}
$$

Thus, $B^{h}[\psi]$ tends to 0 as $h \longrightarrow 0$.

By the change of variable $t=t+h$, we have

$$
\begin{aligned}
& \int_{[0, T] \times \Omega \times B_{R}} f^{h}\left[\frac{G^{\prime}(f(t+h)) u(t+h)-G^{\prime}(f(t)) u(t)}{h}\right] \mathrm{d} t \mathrm{~d} x \mathrm{~d} v \\
& =X_{1}^{h}+X_{2}^{h}+X_{3}^{h},
\end{aligned}
$$

where 


$$
\begin{aligned}
& X_{1}^{h}=\int_{[0, T] \times \Omega \times B_{R}} f^{h}(t-h)\left[\frac{G^{\prime}(f(t)) u(t)-G^{\prime}(f(t-h)) u(t-h)}{h}\right] \mathrm{d} t \mathrm{~d} x \mathrm{~d} v, \\
& X_{2}^{h}=\int_{]-h, 0\left[\times \Omega \times B_{R}\right.} f^{h}(t-h)\left[\frac{G^{\prime}(f(t)) u(t)-G^{\prime}(f(t-h)) u(t-h)}{h}\right] \mathrm{d} t \mathrm{~d} x \mathrm{~d} v, \\
& X_{3}^{h}=-\int_{] T-h, T\left[\times \Omega \times B_{R}\right.} f^{h}(t-h)\left[\frac{G^{\prime}(f(t)) u(t)-G^{\prime}(f(t-h)) u(t-h)}{h}\right] \mathrm{d} t \mathrm{~d} x \mathrm{~d} v .
\end{aligned}
$$

Recalling the definition of $f^{h}$, we get

$X_{2}^{h}=\int_{]-h, 0\left[\times \Omega \times B_{R}\right.} f_{0} G^{\prime}\left(f_{0}\right)\left[\frac{u(t)-u(t-h)}{h}\right] \mathrm{d} t \mathrm{~d} x \mathrm{~d} v$.

$$
\begin{aligned}
\left|X_{3}^{h}\right| & \leq M\left|G^{\prime}(M)\right|\left[\frac{1}{h} \int_{[0, h] \times \Omega \times B_{R}} u(t+T-h)\right. \\
& \left.+\frac{1}{h} \int_{[0, h] \times \Omega \times B_{R}} u(t+T-2 h)\right],
\end{aligned}
$$

Thus,

$$
\lim _{h \longrightarrow 0} X_{2}^{h}=0 .
$$

Besides,

and since $\sup p(u) \subset[-T, T]$,

$$
\lim _{h \longrightarrow 0}\left|X_{3}^{h}\right| \leq M\left|G^{\prime}(M)\right|[u(T)+u(T)]=0 .
$$

We also have

$$
\begin{aligned}
& X_{1}^{h}=\int_{[0, T] \times \Omega \times B_{R}}\left(f^{h}(t-h)-f\right)\left[\frac{G^{\prime}(f(t)) u(t)-G^{\prime}(f(t-h)) u(t-h)}{h}\right] \mathrm{d} t \mathrm{~d} x \mathrm{~d} v \\
& +\int_{[0, T] \times \Omega \times B_{R}} f\left[\frac{G^{\prime}(f(t)) u(t)-G u \prime(f(t-h))(t-h)}{h}\right] \mathrm{d} t \mathrm{~d} x \mathrm{~d} v .
\end{aligned}
$$

But

$$
\begin{gathered}
\left|\int_{[0, T] \times \Omega \times B_{R}}\left(f^{h}(t-h)-f\right)\left[\frac{G^{\prime}(f(t)) u(t)-G^{\prime}(f(t-h)) u(t-h)}{h}\right] \mathrm{d} t \mathrm{~d} x \mathrm{~d} v\right| \leq \\
\left\|f^{h}(t-h)-f\right\|_{L^{\infty}} \int_{[0, T] \times \Omega \times B_{R}}\left|\frac{G^{\prime}(f(t)) u(t)-G^{\prime}(f(t-h)) u(t-h)}{h}\right|^{p} \mathrm{~d} t \mathrm{~d} x \mathrm{~d} v, \\
\lim _{h \rightarrow 0}\left\|f^{h}(t-h)-f\right\|_{L^{\infty}}=0,
\end{gathered}
$$

and then by the dominated convergence theorem, we have and using (232) and (233), we obtain that

$$
\begin{aligned}
& \lim _{h \longrightarrow 0} \int_{[0, T] \times \Omega \times B_{R}}\left|\frac{G^{\prime}(f(t)) u(t)-G^{\prime}(f(t-h)) u(t-h)}{h}\right|^{p} \mathrm{~d} t \mathrm{~d} x \mathrm{~d} v \\
& =\int_{[0, T] \times \Omega \times B_{R}}\left|\partial_{t}\left(G^{\prime}(f(t)) u(t)\right)\right|^{p}<\infty,
\end{aligned}
$$

$$
\lim _{h \longrightarrow 0} \int_{[0, T] \times \Omega \times B_{R}}\left(f^{h}(t-h)-f\right)\left[\frac{G^{\prime}(f(t)) u(t)-G^{\prime}(f(t-h)) u(t-h)}{h}\right] \mathrm{d} t \mathrm{~d} x \mathrm{~d} v=0 .
$$

Next, the equality 


$$
\begin{aligned}
& \lim _{h \longrightarrow 0} \int_{[0, T] \times \Omega \times B_{R}} f^{h}\left[\frac{G^{\prime}(f(t+h)) u(t+h)-G^{\prime}(f(t)) u(t)}{h}\right] \mathrm{d} t \mathrm{~d} x \mathrm{~d} v \\
& =\lim _{h \longrightarrow 0} \int_{[0, T] \times \Omega \times B_{R}} f\left[\frac{G^{\prime}(f(t)) u(t)-G^{\prime}(f(t-h)) u(t-h)}{h}\right] \mathrm{d} t \mathrm{~d} x \mathrm{~d} v,
\end{aligned}
$$

follows by recalling (229), (227), and (234), and accordingly,

$$
\begin{aligned}
& \lim _{h \longrightarrow 0} \int_{0}^{T} \int_{\Omega \times B_{R}} f\left[\frac{G^{\prime}(f) u(t)-G^{\prime}(f(t-h)) u(t-h)}{h}\right] \mathrm{d} t \mathrm{~d} x \mathrm{~d} v \\
& +\int_{\Omega \times B_{R}} f_{0} G^{\prime}\left(f_{0}\right) u(0) \mathrm{d} x \mathrm{~d} v \\
& =\int_{0}^{T} \int_{\Omega \times B_{R}}\left\langle f \sigma, \nabla_{v} G^{\prime}(f)\right\rangle u(t) \mathrm{d} t \mathrm{~d} x \mathrm{~d} v .
\end{aligned}
$$

We notice that

$$
\begin{aligned}
& f\left[\frac{G^{\prime}(f) u(t)-G^{\prime}(f(t-h)) u(t-h)}{h}\right] \\
& =f G^{\prime}(f) \partial_{t}^{-h} u(t)+\frac{1}{h} f u(t-h)\left[G^{\prime}(f)-G^{\prime}(f(t-h))\right],
\end{aligned}
$$

where $\partial_{t}^{-h} u(t)=u(t)-u(t-h) / h$, and since $G \in C^{1}$ is convex, then

$$
G^{*}\left(G^{\prime}(f)\right)-G^{*}\left(G^{\prime}(f(t-h))\right) \leq f\left(G^{\prime}(f)-G^{\prime}(f(t-h))\right) .
$$

Accordingly,

$$
\begin{aligned}
& f\left[\frac{G^{\prime}(f) u(t)-G^{\prime}(f(t-h)) u(t-h)}{h}\right] \\
& \geq f G^{\prime}(f) \partial_{t}^{-h} u(t)+\frac{1}{h} u(t-h) \\
& {\left[G^{*}\left(G^{\prime}(f)\right)-G^{*}\left(G^{\prime}(f(t-h))\right)\right],}
\end{aligned}
$$

and after integration, we get

$$
\begin{aligned}
& \int_{0}^{T} \int_{\Omega \times B_{R}} f\left[\frac{G^{\prime}(f) u(t)-G^{\prime}(f(t-h)) u(t-h)}{h}\right] \mathrm{d} t \mathrm{~d} x \mathrm{~d} v \\
& \geq \int_{0}^{T} \int_{\Omega \times B_{R}} f G^{\prime}(f) \partial_{t}^{-h} u(t) \mathrm{d} t \mathrm{~d} x \mathrm{~d} v \\
& +\frac{1}{h} \int_{0}^{T} \int_{\Omega \times B_{R}} u(t-h)\left[G^{*}\left(G^{\prime}(f)\right)\right. \\
& \left.\quad-G^{*}\left(G^{\prime}(f(t-h))\right)\right] \mathrm{d} t \mathrm{~d} x \mathrm{~d} v
\end{aligned}
$$

Since $\sup p(u) \subset[-T, T], u(t)=0$ for $t-h \in[T-h, T]$, and then, we obtain
$\int_{0}^{T} \int_{\Omega \times B_{R}} f\left[\frac{G^{\prime}(f) u(t)-G^{\prime}(f(t-h)) u(t-h)}{h}\right] \mathrm{d} t \mathrm{~d} x \mathrm{~d} v \geq$

$\int_{0}^{T} \int_{\Omega \times B_{R}}\left[f G^{\prime}(f)-G^{*}\left(G^{\prime}(f)\right)\right] \partial_{t}^{-h} u(t) \mathrm{d} t \mathrm{~d} x \mathrm{~d} v+$

$-\frac{1}{h} \int_{0}^{T} \int_{\Omega \times B_{R}} G^{*}\left(G^{\prime}(f(t-h))\right) u(t-h) \mathrm{d} t \mathrm{~d} x \mathrm{~d} v$.

Tending $h$ to 0 in the relation above enables us to have

$\lim _{h \longrightarrow 0} \int_{0}^{T} \int_{\Omega \times B_{R}} f\left[\frac{G^{\prime}(f) u(t)-G^{\prime}(f(t-h)) u(t-h)}{h}\right] \mathrm{d} t \mathrm{~d} x \mathrm{~d} v$

$\geq \int_{0}^{T} \int_{\Omega \times B_{R}}\left[f G^{\prime}(f)-G^{*}\left(G^{\prime}(f)\right)\right] u^{\prime}(t) \mathrm{d} t \mathrm{~d} x \mathrm{~d} v$

$-\int_{\Omega \times B_{R}} G^{*}\left(G^{\prime}\left(f_{0}\right)\right) u(0) \mathrm{d} x \mathrm{~d} v$.

Moreover, expressions (236) and (242) give

$\int_{\Omega \times B_{R}}\left[f_{0} G^{\prime}\left(f_{0}\right)-G^{*}\left(G^{\prime}\left(f_{0}\right)\right)\right] u(0) \mathrm{d} x \mathrm{~d} v$

$+\int_{[0, T] \times \Omega \times B_{R}}\left[f G^{\prime}(f)-G^{*}\left(G^{\prime}(f)\right)\right] u^{\prime}(t) \mathrm{d} t \mathrm{~d} x \mathrm{~d} v$

$\leq \int_{[0, T] \times \Omega \times B_{R}}\left\langle f \sigma, \nabla_{\nu} G^{\prime}(f)\right\rangle u(t) \mathrm{d} t \mathrm{~d} x \mathrm{~d} v$.

So, tending $R$ to $\infty$ in (243), we obtain Lemma 15 .

Now, we derive (185) by combining Lemmas 13-15.

So, let us show that the sequence $\left(\operatorname{div}_{v}\left(f^{h} \sigma^{h}\right)\right)_{h}$ converges weakly to $\operatorname{div}_{v}(f \sigma)$ in $\left[C_{c}^{\infty}\left(\mathbb{R} \times \Omega \times \mathbb{R}^{d}\right)\right]^{\prime}$ and $\operatorname{div}_{v}(f \sigma)=\operatorname{div}_{v}\left(f \nabla c^{*}\left(\nabla_{v} G^{\prime}(f)\right)\right)$ in the weak sense.

So, fix $T>0$ and let $\psi \in C_{c}^{\infty}\left(\mathbb{R} \times \Omega \times \mathbb{R}^{d}\right)$ such that $\sup p \psi(., x, v) \subset[-T, T]$.

We have

$\lim _{h \rightarrow 0} \int_{0}^{T} \int_{\Omega \times \mathbb{R}^{d}}\left\langle f^{h} \nabla c^{*}\left(\nabla G^{\prime}\left(f^{h}\right)\right)-f \sigma, \nabla_{v} \psi\right\rangle \mathrm{d} t \mathrm{~d} x \mathrm{~d} v=0$.

Indeed,

$$
\begin{aligned}
& \int_{0}^{T} \int_{\Omega \times \mathbb{R}^{d}}\left\langle f^{h} \nabla c^{*}\left(\nabla_{v} G^{\prime}\left(f^{h}\right)\right)-f \sigma, \nabla_{v} \psi\right\rangle \mathrm{d} t \mathrm{~d} x \mathrm{~d} v \\
& =\int_{0}^{T} \int_{\Omega \times \mathbb{R}^{d}} f\left\langle\nabla c^{*}\left(\nabla G^{\prime}\left(f^{h}\right)\right)-\sigma, \nabla_{v} \psi\right\rangle \mathrm{d} t \mathrm{~d} x \mathrm{~d} v \\
& +\int_{0}^{T} \int_{\Omega \times \mathbb{R}^{d}}\left(f^{h}-f\right)\left\langle\nabla c^{*}\left(\nabla_{v} G^{\prime} f^{h}\right), \nabla_{v} \psi\right\rangle \mathrm{d} t \mathrm{~d} x \mathrm{~d} v .
\end{aligned}
$$

We use (i), (iii), and the fact that $\nabla_{v} \psi \in L^{p}([0, T] \times \Omega \times$ $\left.\mathbb{R}^{d}\right) \quad$ and $\left(\nabla c^{*}\left(\nabla_{v} G^{\prime}\left(f^{h}\right)\right)\right)_{h} \quad$ is bounded in $L^{q}\left([0, T] \times \Omega \times \mathbb{R}^{d}\right)$, and we tend $h$ to 0 in (245) to obtain (244). 
Let us show that $\operatorname{div}_{v}(f \sigma)=\operatorname{div}_{v}\left(f \nabla c^{*}\left(\nabla_{v} G^{\prime}\right)(f)\right)$ in the weak sense. So, let $\varepsilon>0$ and $\psi \in C_{c}^{\infty}\left(\Omega \times \mathbb{R}^{d}\right)$ be a test function and $u \in C_{c}^{\infty}(\mathbb{R}), u \geq 0$, with $\operatorname{supp}(u) \subset[-T, T]$. Define $\omega_{\varepsilon}(t, x, v)=G^{\prime}(f)-\varepsilon \psi$.

Since $c^{*}$ is convex and $f^{h} \geq 0$, we have $\int_{[0, T] \times \Omega \times \mathbb{R}^{\mathbb{d}^{d}}} f^{h}\left\langle\nabla c^{*}\left(\left(\nabla_{\nu} G^{\prime}\left(f^{h}\right)\right)-\nabla c^{*}\left(\nabla \omega_{\varepsilon}\right), \nabla_{\nu} G^{\prime}\left(f^{h}\right)-\nabla \omega_{\varepsilon}\right\rangle u(t) \geq 0\right.$.

Splitting (246) as follows,

$$
\begin{aligned}
& \int_{[0, T] \times \Omega \times \mathbb{R}^{d}} f^{h}\left\langle\nabla c^{*}\left(\nabla_{\nu} G^{\prime}\left(f^{h}\right)\right)-\nabla c^{*}\left(\nabla \omega_{\varepsilon}\right), \nabla G^{\prime}\left(f^{h}\right)-\nabla \omega_{\varepsilon}\right\rangle u(t) \\
& =\int_{[0, T] \times \Omega \times \mathbb{R}^{d}} f^{h}\left\langle\nabla c^{*}\left(\nabla_{\nu} G^{\prime}\left(f^{h}\right)\right)-\nabla c^{*}\left(\nabla \omega_{\varepsilon}\right), \nabla_{\nu} G^{\prime}\left(f^{h}\right)-\nabla_{\nu} G^{\prime}(f)\right\rangle u(t) \\
& +\int_{[0, T] \times \Omega \times \mathbb{R}^{d}} f^{h}\left\langle\nabla c^{*}\left(\nabla_{\nu} G^{\prime}\left(f^{h}\right)\right)-\nabla c^{*}\left(\nabla \omega_{\varepsilon}\right), \nabla_{\nu} G^{\prime}(f)-\nabla \omega_{\varepsilon}\right\rangle u(t),
\end{aligned}
$$

we derive from (185) that

Consequently,

$$
\begin{aligned}
& \lim _{h \longrightarrow 0} \int_{[0, T] \times \Omega \times \mathbb{R}^{d}} f^{h}\left\langle\nabla c^{*}\left(\nabla_{\nu} G^{\prime}\left(f^{h}\right)\right)\right. \\
& \left.\quad-\nabla c^{*}\left(\nabla \omega_{\varepsilon}\right), \nabla_{\nu} G^{\prime}\left(f^{h}\right)-\nabla G^{\prime}(f)\right\rangle u(t)=0 .
\end{aligned}
$$

$$
\begin{aligned}
& \lim _{h \longrightarrow 0} \int_{[0, T] \times \Omega \times \mathbb{R}^{d}} f^{h}\left\langle\nabla c^{*}\left(\nabla_{v} G^{\prime}\left(f^{h}\right)\right)-\nabla c^{*}\left(\nabla \omega_{\varepsilon}\right), \nabla_{\nu} G^{\prime}\left(f^{h}\right)-\nabla \omega_{\varepsilon}\right\rangle u(t) \\
& =\int_{[0, T] \times \Omega \times \mathbb{R}^{d}}\left\langle f \sigma-f \nabla c^{*}\left(\nabla \omega_{\varepsilon}\right), \nabla_{\nu} G^{\prime}(f)-\nabla \omega_{\varepsilon}\right\rangle u(t) \geq 0 .
\end{aligned}
$$

We divide (249) by $\varepsilon$, and we tend $\varepsilon$ to 0 and have

$$
\int_{[0, T] \times \Omega \times \mathbb{R}^{d}}\left\langle f \sigma-f \nabla c^{*}\left(\nabla_{v} G^{\prime}(f)\right), \nabla_{v} \psi u(t) \leq 0\right.
$$
obtain

Replacing $\psi$ by $-\psi$ in the previous relationship, we

$$
\int_{[0, T] \times \Omega \times \mathbb{R}^{d}}\left\langle f \sigma-f \nabla c^{*}\left(\nabla_{\nu} G^{\prime}(f)\right), \nabla_{\nu} \psi\right\rangle u(t)=0 .
$$

We conclude that $\left(\operatorname{div}_{v}\left(f^{h} \nabla c^{*}\left(\nabla_{v} G^{\prime}\left(f^{h}\right)\right)\right)\right)$ converges weakly to $\operatorname{div}_{v}\left(f \nabla c^{*}\left(\nabla_{v} G^{\prime}(f)\right)\right)$ in $\left[C_{c}^{\infty}\left(\mathbb{R} \times \Omega \times \mathbb{R}^{d}\right)\right]^{\prime}$, and then, the proof of Theorem 2 is complete.

4.5. Existence of Solutions. Now, we combine the weak convergence of the sequence $\left(f^{h}\right)_{h}$ and that of the nonlinear term $\operatorname{div}_{v}\left(f^{h} \nabla c^{*}\left(\nabla_{v} G^{\prime}\left(f^{h}\right)\right)\right)$ to prove that the kinetic equation (1) admits a weak solution $f$ in the sense of Definition 1.

Theorem 3. Let $f_{0}$ be a density of probability on $\Omega \times \mathbb{R}^{d}$ such that $f_{0}+\left(1 / f_{0}\right) \in L^{\infty}\left(\Omega \times \mathbb{R}^{d}\right)$, $\int_{\Omega \times \mathbb{R}^{d}}|v|^{q} f_{0} \mathrm{~d} x \mathrm{~d} v<\infty$, and $\int_{\Omega \times \mathbb{R}^{d}} G\left(f_{0}\right) \mathrm{d} x \mathrm{~d} v<\infty$ with $G$ and $c$ satisfying, respectively, $H_{G}$ and $H_{c}$.

Then, for any test function $\psi \in C_{c}^{\infty}\left(\mathbb{R} \times \Omega \times \mathbb{R}^{d}\right)$ such that $\operatorname{supp} \psi(., x, v) \subset[-T, T]$, we have

$$
\begin{aligned}
& \int_{[0, T] \times \Omega \times \mathbb{R}^{d}} f\left(\partial_{t} \psi+v \cdot \nabla_{x} \psi-\nabla_{\nu} \psi \cdot \nabla c^{*}\left(\nabla_{\nu} G^{\prime}(f)\right)\right) \mathrm{d} t \mathrm{~d} x \mathrm{~d} v \\
& =-\int_{\Omega \times \mathbb{R}^{d}} f_{0} \psi(0) \mathrm{d} x \mathrm{~d} v,
\end{aligned}
$$

where $f$ is the limit function obtained in Proposition 3 .

Proof. Fix $T>0$ and let $\psi \in C_{c}^{\infty}\left(\mathbb{R} \times \Omega \times \mathbb{R}^{d}\right)$ be a test function such that $\operatorname{supp} \psi(., x, v) \subset[-T, T]$. Integrating (17) over $[0, T] \times \Omega \times \mathbb{R}^{d}$, we obtain

$$
\begin{aligned}
& \sum_{k=1}^{T / h} \int_{t_{k-1}}^{t_{k}} \int_{\Omega \times \mathbb{R}^{d}} \frac{f_{k}^{h}-f_{k-1}^{h}}{h} \psi\left(t, x+\left(t-t_{k-1}\right) v, v\right) \mathrm{d} \mathrm{d} \mathrm{d} x \mathrm{~d} v \\
& +\sum_{k=1}^{T / h} \int_{t_{k-1}}^{t_{k}} \int_{\Omega \times \mathbb{R}^{d}} f_{k-1}^{h} v \cdot \nabla_{x} \psi\left(t, x+\left(t-t_{k-1}\right) v, v\right) \mathrm{d} t \mathrm{~d} x \mathrm{~d} v \\
& +\sum_{k=1}^{T / h} \int_{t_{k-1}}^{t_{k}} \int_{\Omega \times \mathbb{R}^{d}} f_{k}^{h} \nabla_{v} \psi\left(t, x+\left(t-t_{k-1}\right) v, v\right) \cdot \nabla c^{*}\left(\nabla_{v} G^{\prime}\left(f_{k}^{h}\right)\right)=B^{h}[\psi],
\end{aligned}
$$

where $B^{h}[\psi]$ tends to 0 as $h \longrightarrow 0$.

We observe that 
$\sum_{k=1}^{T / h} \int_{t_{k-1}}^{t_{k}} \int_{\Omega \times \mathbb{R}^{d}} \frac{f_{k}^{h}-f_{k-1}^{h}}{h} \psi\left(t, x+\left(t-t_{k-1}\right) v, v\right) \mathrm{d} t \mathrm{~d} x \mathrm{~d} v$

$=\int_{[0, T] \times \Omega \times \mathbb{R}^{d}} f^{h}\left[\frac{\psi(t-h, x, v)-\psi(t, x, v)}{h}\right] \mathrm{d} t \mathrm{~d} x \mathrm{~d} v$

$-\frac{1}{h} \int_{0}^{h} \int_{\Omega \times \mathbb{R}^{d}} f_{0}(x-t v, v) \psi(t-h, x, v) \mathrm{d} t \mathrm{~d} x \mathrm{~d} v$,

$\sum_{k=1}^{T / h} \int_{t_{k-1}}^{t_{k}} \int_{\Omega \times \mathbb{R}^{d}} f_{k-1}^{h} v \cdot \nabla_{x} \psi\left(t, x+\left(t-t_{k-1}\right) v, v\right) \mathrm{d} t \mathrm{~d} x \mathrm{~d} v$

$=\int_{[0, T] \times \Omega \times \mathbb{R}^{d}} f^{h} v \cdot \nabla_{x} \psi \mathrm{d} t \mathrm{~d} x \mathrm{~d} v$.

Hence,

$$
\begin{aligned}
& \sum_{k=1}^{T / h} \int_{t_{k-1}}^{t_{k}} \int_{\Omega \times \mathbb{R}^{d}} f_{k}^{h} \nabla_{v} \psi\left(t, x+\left(t-t_{k-1}\right) v, v\right) \\
& \cdot \nabla c^{*}\left(\nabla_{v} G^{\prime}\left(f_{k}^{h}\right)\right) \mathrm{d} t \mathrm{~d} x \mathrm{~d} v \\
& =\int_{[0, T] \times \Omega \times \mathbb{R}^{d}} f^{h} \nabla_{v} \cdot \psi \cdot \nabla c^{*}\left(\nabla_{v} G^{\prime}\left(f^{h}\right)\right) \mathrm{d} t \mathrm{~d} x \mathrm{~d} v \\
& -\int_{[0, h] \times \Omega \times \mathbb{R}^{d}} f_{0}(x-t v, v) \nabla_{v} \psi(t, x, v) \\
& \quad \cdot \nabla c^{*}\left(\nabla G^{\prime}\left(f_{0}(x-t v, v)\right)\right) \mathrm{d} t \mathrm{~d} x \mathrm{~d} v .
\end{aligned}
$$

We combine (254), (255), and (256) to obtain

$$
\begin{aligned}
& B^{h}[\psi]=-\int_{[0, T] \times \Omega \times \mathbb{R}^{d}} f^{h} \partial_{t}^{-h} \psi(t, x, v) \mathrm{d} t \mathrm{~d} x \mathrm{~d} v \\
& -\frac{1}{h} \int_{0}^{h} \int_{\Omega \times \mathbb{R}^{d}} f_{0}(x-t v, v) \psi(t-h, x, v) \mathrm{d} t \mathrm{~d} x \mathrm{~d} v \\
& +\int_{[0, T] \times \Omega \times \mathbb{R}^{d}} f^{h} \nabla_{v} \psi \cdot \nabla c^{*}\left(\nabla_{v} G^{\prime}\left(f^{h}\right)\right) \mathrm{d} t \mathrm{~d} x \mathrm{~d} v \\
& -\int_{[0, T] \times \Omega \times \mathbb{R}^{d}} f^{h} v \cdot \nabla_{x} \psi \mathrm{d} t \mathrm{~d} x \mathrm{~d} v \\
& -\int_{[0, h] \times \Omega \times \mathbb{R}^{d}} f_{0}(x-t v, v) \nabla_{v} \psi(t, x, v) \\
& \cdot \nabla c^{*}\left(\nabla_{v} G^{\prime}\left(f_{0}(x-t v, v)\right)\right) \mathrm{d} t \mathrm{~d} x \mathrm{~d} v .
\end{aligned}
$$

Here,

$$
\partial_{t}^{-h} \psi(t, x, v)=\frac{\psi(t, x, v)-\psi(t-h, x, v)}{h} .
$$

Finally, we use the weak convergence of $\left(f^{h}\right)_{h}$ to $f$, the weak convergence of $f^{h} \nabla c^{*}\left(\nabla_{v} G^{\prime}\left(f^{h}\right)\right)$ to $f \nabla c^{*}\left(\nabla_{v} G^{\prime}(f)\right)$, and the uniform convergence of $\partial_{t}^{h} \psi(., x, v)$ to $\partial_{t} \psi(., x, v)$ on the compact set $[0, T]$ when $h$ tend to 0 , to derive the following equality:

$$
\begin{aligned}
& -\int_{[0, T] \times \Omega \times \mathbb{R}^{d}} f \partial_{t} \psi \mathrm{d} t \mathrm{~d} x \mathrm{~d} v-\int_{\Omega \times \mathbb{R}^{d}} f_{0} \psi(0) \mathrm{d} x \mathrm{~d} v \\
& -\int_{[0, T] \times \Omega \times \mathbb{R}^{d}} f v \cdot \nabla_{x} \psi \mathrm{d} t \mathrm{~d} x \mathrm{~d} v+\int_{[0, T] \times \Omega \times \mathbb{R}^{d}} \nabla_{v} \psi \\
& \quad \cdot \nabla c^{*}\left(\nabla_{v} G^{\prime}(f)\right) f \mathrm{~d} t \mathrm{~d} x \mathrm{~d} v=0,
\end{aligned}
$$

i.e.,

$$
\begin{aligned}
& \int_{[0, T] \times \Omega \times \mathbb{R}^{d}}\left[\partial_{t} \psi+v \cdot \nabla_{x} \psi-\nabla_{v} \psi \cdot \nabla c^{*}\left(\nabla_{v} G^{\prime}(f)\right)\right] f \mathrm{~d} t \mathrm{~d} x \mathrm{~d} v \\
& =-\int_{\Omega \times \mathbb{R}^{d}} f_{0} \psi(0) \mathrm{d} x \mathrm{~d} v .
\end{aligned}
$$

In conclusion, we prove that $f$ solves (1) in the weak sense.

\section{Numerical Implementation}

In this section, we use the iterative operator-splitting methods as in [10] to construct an approximate weak solution of the kinetic equation (1) as stated in Section 2.1, and we establish the rate of convergence of our algorithm to the exact solution of the kinetic equation. The scheme consisting after fixing a time step $h>0$ and denoting $t_{k}=h k$, with $k \in \mathbb{N}^{*}$, in defining the approximate solution $u^{i}=u^{i}(t, x, v)$, $(t, x, v) \in\left[t_{k}, t_{k+1}\right) \times \Omega \times \mathbb{R}^{d}$ of $(1)$ is as follows:

$$
\begin{cases}\frac{\partial u^{i}}{\partial t}+v \cdot \nabla_{x} u^{i}=\operatorname{div}_{v}\left\{u^{i-1} \nabla c^{*}\left[\nabla_{v}\left(G^{\prime}\left(u^{i-1}\right)\right)\right]\right\}, & \text { in }\left[t_{k}, t_{k+1}\right) \times \Omega \times \mathbb{R}^{d}, \\ \frac{\partial u^{i+1}}{\partial t}+v \cdot \nabla_{x} u^{i}=\operatorname{div}_{v}\left\{u^{i+1} \nabla c^{*}\left[\nabla_{v}\left(G^{\prime}\left(u^{i+1}\right)\right)\right]\right\}, & \text { in }\left[t_{k}, t_{k+1}\right) \times \Omega \times \mathbb{R}^{d},\end{cases}
$$


where $i=1 ; 3 ; 5 ; \ldots ; 2 j+1$, with $j \in \mathbb{N}^{*}$ and $u^{i}\left(t_{k}, x, v\right)=f_{k}^{h}(x, v), u^{i+1}\left(t_{k}, x, v\right)=f_{k}^{h}(x, v) ; f_{k}^{h}$ denotes the approximate solution of (1) at $t=t_{k}$ defined in Section 2.1 .

Remark 2. By fixing $u^{i-1}$, we obtain

$$
\begin{aligned}
& u^{i}(t, x, v)=f_{k}^{h}\left(x-\left(t-t_{k}\right) v, v\right) \\
& +\int_{t_{k}}^{t} \operatorname{div}_{v}\left\{u^{i-1}\left(s, x-\left(s-t_{k}\right) v, v\right) \nabla c^{*}\right. \\
& \left.\quad\left(\nabla_{v} G^{\prime}\left(u^{i-1}\left(s, x-\left(s-t_{k}\right) v, v\right)\right)\right)\right\} \mathrm{d} s .
\end{aligned}
$$

By fixing $u^{i}$, we obtain $u^{i+1}$ as follows:

$$
u^{i+1}(t, x, v)=\chi^{i+1}(t, x) U^{x, i+1}(t, v)
$$

where

$$
\begin{aligned}
& \chi^{i+1}(t, x)=\int_{\mathbb{R}^{d}} u^{i}(t-h, x-h v, v) \mathrm{d} v, \\
& U^{x, i+1} \in \operatorname{Argmin}\left\{\frac{1}{\chi^{i+1}(t, x)} \int_{\mathbb{R}^{d}}\right. \\
& \left.G\left(\chi^{i+1}(t, x) U\right) \mathrm{d} v+h W_{c}^{h}\left(U, V^{x, i+1}\right)\right\},
\end{aligned}
$$

with

$$
V^{x, i+1}(t, v)=\frac{u^{i}(t-h, x-h v, v)}{\chi^{i+1}(t, x)} .
$$

Note that, for $t=t_{k}$ and for $i=1 ; 3 ; \ldots ; 2 j+1, j \in \mathbb{N}^{*}$, we obtain $u^{i}\left(t_{k}, x, v\right)=f_{k}^{h}(x, v), u^{i+1}\left(t_{k}, x, v\right)=f_{k}^{h}(x, v)$, $\chi^{i+1}\left(t_{k}, x\right)=\rho_{k}^{h}(x), \quad V^{x, i+1}\left(t_{k}, v\right)=G_{k}^{x h}(v), \quad$ and $U^{x, i+1}\left(t_{k}, v\right)=F_{k}^{x h}(v)$, where $\rho_{k}^{h}, G_{k}^{x h}, F_{k}^{x h}$, and $f_{k}^{h}$ are defined as in the descent algorithm (see Section 2.1).

5.1. Error Analysis. An estimate of the rate of convergence of $u^{i}$ to the exact solution of (1) is given as follows.

Theorem 4. Assume that $f_{0}, c$, and $G$ satisfy, respectively, $\left(H_{f_{0}}\right),\left(H_{c}\right)$, and $\left(H_{G}\right)$, and $\operatorname{supp} f_{0}(x,.) \subset B_{R}$, for all $x \in \Omega$; $\nabla c^{*}(0)=0$. Then, we obtain the following error estimation:

$$
\left\|e^{j}(t)\right\|_{\infty, L^{q}\left(\Omega \times B_{R}\right)} \leq(K h)^{j-1}\left\|e^{0}\right\|_{\infty, L^{q}\left(\Omega \times B_{R}\right)},
$$

where $e^{j}(t, x, v)=f(t, x, v)-u^{j}(t, x, v), j \in \mathbb{N}^{*}$ is the error between the exact solution $f$ and the approximate solution $u^{j}$ and $q=p /(p-1) \leq p$ and $e^{0}=f-f_{k}^{h}$, and $K$ is a constant depending on $N, M, R$, and $G^{\prime}$.
Proof. We use the error function $e^{i}=f-u^{i}$ and the linearized equation of (1) via iterative splitting methods. Then, we have for $i=1 ; 3 ; \ldots ; 2 j+1$ :

$$
\begin{aligned}
& \frac{\partial e^{i}}{\partial t}+\left(v \cdot \nabla_{x} e^{i}\right)=\operatorname{div}_{v}\left(u^{i-1} \nabla c^{*}\left(\nabla_{v} G^{\prime}\left(u^{i-1}\right)\right)\right) e^{i-1} \\
& \quad+\operatorname{div}_{v}\left(u^{i-1} D^{2} c^{*}\left(\nabla_{v} G^{\prime}\left(u^{i-1}\right)\right) \circ D_{v}^{2} G^{\prime}\left(u^{i-1}\right)\right) e^{i-1}+o\left(\left\|e^{i-1}\right\|\right) .
\end{aligned}
$$

Since supp $f_{0}(x,.) \subset B_{R}$ and $N \leq f_{0} \leq M$, then Lemma 16 gives that $\operatorname{supp} u^{i}(t, x,.) \subset B_{R}$ and $D^{2} c^{*}\left(\nabla_{v} G^{\prime}\left(u^{i-1}\right)\right) \circ \mathrm{D}_{\mathrm{v}}^{2} \mathrm{G}^{\prime}\left(\mathrm{u}^{\mathrm{i}-1}\right) \in \mathrm{L}^{\infty}\left(\left[\mathrm{t}_{\mathrm{k}}, \mathrm{t}_{\mathrm{k}+1}\right] \times \Omega \times \mathrm{B}_{\mathrm{R}}\right)$ and

$$
\left\|D^{2} c^{*}\left(\nabla_{v} G^{\prime}\left(u^{i-1}\right)\right) \circ D_{v}^{2} G^{\prime}\left(u^{i-1}\right)\right\|_{L^{\infty}\left(\left[t_{k}, t_{k+1}\right] \times \Omega \times B_{R}\right)} \leq 1+\frac{M}{N} .
$$

We conclude that $\operatorname{div}_{v}\left(u^{i-1} \nabla c^{*}\left(\nabla_{v} G^{\prime}\left(\left(u^{i-1}\right)\right)\right) \in L^{q}(\Omega \times\right.$ $\left.B_{R}\right)$ and $\operatorname{div}_{v}\left(u^{i-1} D^{2} c^{*}\left(\nabla_{v} G^{\prime}\left(u^{i-1}\right)\right) \circ D_{v}^{2} G^{\prime}\left(u^{i-1}\right)\right) \in L^{q}\left(\Omega \times B_{R}\right)$.

Thus,

$$
\begin{gathered}
\left\|\operatorname{div}_{x}\left(v u^{i-1}\right)\right\|_{\infty, L^{q}\left(\Omega \times B_{R}\right)} \leq K_{1}, \\
\left\|\operatorname{div}_{v}\left(u^{i-1} \nabla c^{*}\left(\nabla_{v} G^{\prime}\left(u^{i-1}\right)\right)\right)\right\|_{\infty, L^{q}\left(\Omega \times B_{R}\right)} \leq K_{2}, \\
\left\|\operatorname{div}_{v}\left(u^{i-1} D^{2} c^{*}\left(\nabla_{v} G^{\prime}\left(u^{i-1}\right)\right) \circ D_{v}^{2} G^{\prime}\left(u^{i-1}\right)\right)\right\|_{\infty, L^{q}\left(\Omega \times B_{R}\right)} \leq K_{3},
\end{gathered}
$$

where $K_{1}, K_{2}$, and $K_{3}$ are constant depending of $N, M, R$, and $G^{\prime}$. By using (267), we have

$$
\begin{aligned}
& e^{i}(t, x+t v, v)=\int_{t_{k}}^{t} \operatorname{div}_{v}\left(u^{i-1} \nabla c^{*}\left(\nabla_{v} G^{\prime}\left(u^{i-1}\right)\right)\right) e^{i-1} \mathrm{~d} s \\
& +\int_{t_{k}}^{t} \operatorname{div}_{v}\left(u^{i-1} D^{2} c^{*}\left(\nabla_{v} G^{\prime}\left(u^{i-1}\right)\right) \circ D_{v}^{2} G^{\prime}\left(u^{i-1}\right)\right) e^{i-1} \mathrm{~d} s \\
& \quad+\int_{t_{k}}^{t} o\left(\left\|e^{i-1}\right\|\right) \mathrm{d} s .
\end{aligned}
$$

We now use (270) and (271) to obtain

$$
\left\|e^{i}\right\|_{\infty, L^{q}\left(\Omega \times B_{R}\right)} \leq K_{4} h\left\|e^{i-1}\right\|_{\infty, L^{q}\left(\Omega \times B_{R}\right)},
$$

with $K_{4}=K_{2}+K_{3}+1$. As in (272), we have

$$
\begin{gathered}
e^{i+1}(t, x, v)=-\int_{t_{k}}^{t}\left(v \cdot \nabla_{x} u^{i}\right) e_{i} \mathrm{~d} s+\int_{t_{k}}^{t} \operatorname{div}_{v}\left(u^{i+1} \nabla c^{*}\left(\nabla_{v} G^{\prime}\left(u^{i+1}\right)\right)\right) e^{i+1} \mathrm{~d} s \\
+\int_{t_{k}}^{t} \operatorname{div}_{v}\left(u^{i+1} D^{2} c^{*}\left(\nabla_{v} G^{\prime}\left(u^{i+1}\right)\right) \circ D_{v}^{2} G^{\prime}\left(u^{i+1}\right)\right) e^{i+1} \mathrm{~d} s+\int_{t_{k}}^{t} o\left(\left\|e^{i+1}\right\|\right) \mathrm{d} s .
\end{gathered}
$$

Next, from (269), (270), and (271), we derive 
$\left\|e^{i+1}\right\|_{\infty, L^{q}\left(\Omega \times B_{R}\right)} \leq h^{2} K_{1} K_{4}\left\|e^{i-1}\right\|_{\infty, L^{q}\left(\Omega \times B_{R}\right)}+K_{4} h\left\|e^{i+1}\right\|_{\infty, L^{q}\left(\Omega \times B_{R}\right)}$.

Accordingly,

$$
\left\|e^{i+1}\right\|_{\infty, L^{q}\left(\Omega \times B_{R}\right)} \leq K h\left\|e^{i-1}\right\|_{\infty, L^{q}\left(\Omega \times B_{R}\right)},
$$

where $K$ depends on $K_{4}$ and $K_{1}$. Finally, we use (273) and (276) to conclude that

$$
\left\|e^{j}\right\|_{\infty, L^{q}\left(\Omega \times B_{R}\right)} \leq(K h)^{j-1}\left\|e^{0}\right\|_{\infty, L^{q}\left(\Omega \times B_{R}\right)},
$$

for $j \in \mathbb{N}^{*}$
5.2. Numerical Example. In this section, we solves (1), when $d=1, G(t)=t \ln t$, and $c^{*}(t)=t^{2} / 2$, i.e., the following Boltzmann equation:

$$
\frac{\partial f}{\partial t}+v \cdot \partial_{x} f=\partial_{v}^{2} f, \quad f=f(t, x, v),(t, x, v) \in[0, \infty) \times \Omega \times(0, \infty) .
$$

We $\quad$ suppose $\quad \Omega:=(0,1) / \mathbb{Z} .=\{x=\bar{x}+n, \bar{x} \in$ $(0,1), n$ browsing $\mathbb{Z}\}$.

The initial datum $f(0, x, v)=f_{0}(x, v)$ is a probability density on $\Omega \times(0, \infty)$.

Let us show that the function $f$ defined by

$$
\left\{\begin{array}{l}
f(t, x, v)=\bar{x}+\frac{v^{3}}{6}+\frac{11}{12 \sqrt{\pi(4 t+1)}} e^{-\left(v^{2} / 4 t+1\right)}, \quad \text { if }(t, \bar{x}, v) \in[0,+\infty) \times(0,1) \times(0,1), \\
f(t, x, v)=\frac{11}{12 \sqrt{\pi(4 t+1)}} e^{-\left(v^{2} / 4 t+1\right)}, \quad \text { if }(t, \bar{x}, v) \in[0,+\infty) \times(0,1) \times[1,+\infty),
\end{array}\right.
$$

is one solution of the kinetic equation (278). Indeed,

Moreover,

$\frac{\partial f(t, x, v)}{\partial t}+v \cdot \partial_{x} f=\partial_{v}^{2} f, \quad$ in $[0,+\infty) \times \Omega \times(0,+\infty)$,

with the initial datum

$$
\left\{\begin{array}{l}
f_{0}(x, v)=\bar{x}+\frac{v^{3}}{6}+\frac{11}{12 \sqrt{\pi}} e^{-v^{2}}, \quad \text { if }(\bar{x}, v) \in(0,1) \times(0,1), \\
f_{0}(x, v)=\frac{11}{12 \sqrt{\pi}} e^{-v^{2}}, \quad \text { if }(x, v) \in(0,1) \times[1,+\infty) .
\end{array}\right.
$$

$$
\begin{aligned}
& \frac{\partial f}{\partial t}= {\left[\frac{11 v^{2}}{3(4 t+1)^{2} \sqrt{\pi(4 t+1)}}-\frac{11}{6(4 t+1) \sqrt{\pi(4 t+1)}}\right] e^{-\left(v^{2} / 4 t+1\right),} } \\
& v . \partial_{x} f= \begin{cases}v, \quad \text { if }(t, \bar{x}, v) \in[0,+\infty[\times(0,1) \times(0,1), \\
0, \quad \text { if }(t, \bar{x}, v) \in[0,+\infty[\times(0,1) \times[1,+\infty),\end{cases} \\
& \partial_{v}^{2} f=\left\{\begin{array}{l}
v+\left[\frac{11 v^{2}}{3(4 t+1)^{2} \sqrt{\pi(4 t+1)}}-\frac{11}{6(4 t+1) \sqrt{\pi(4 t+1)}}\right] e^{-\left(v^{2} / 4 t+1\right),} \quad \text { if }(t, \bar{x}, v) \in[0,+\infty[\times(0,1) \times(0,1), \\
{\left[\frac{11 v^{2}}{3(4 t+1)^{2} \sqrt{\pi(4 t+1)}}-\frac{11}{6(4 t+1) \sqrt{\pi(4 t+1)}}\right] e^{-\left(v^{2} / 4 t+1\right),} \quad \text { if }(t, \bar{x}, v) \in[0,+\infty[\times(0,1) \times[1,+\infty) .}
\end{array}\right.
\end{aligned}
$$




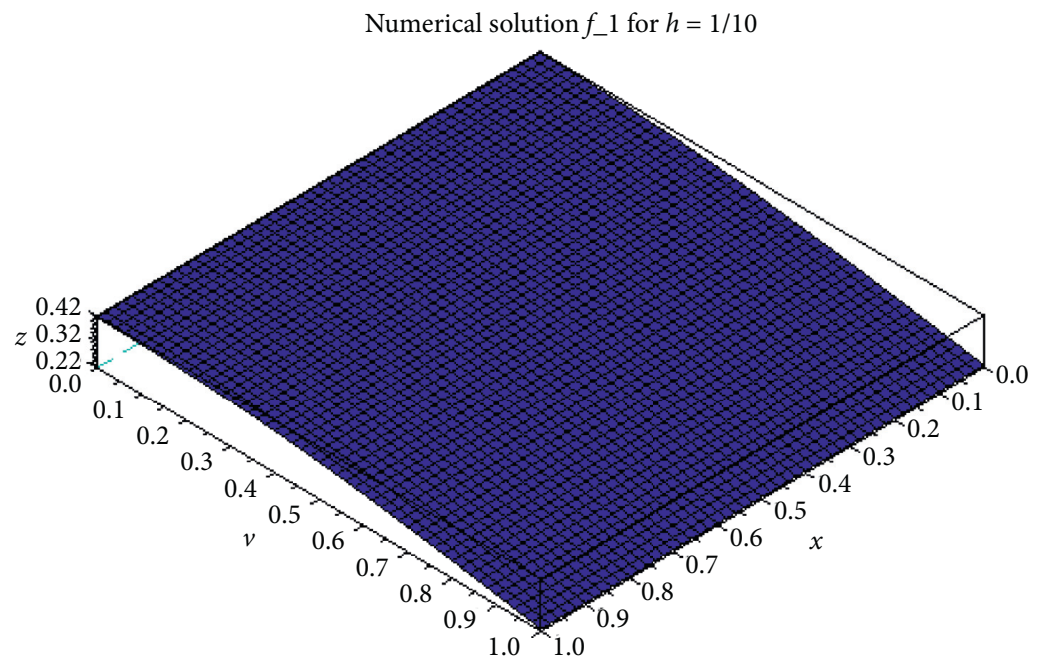

FIgURE 1: Numerical solution $f_{1}^{1 / 10}(x, v)$ for $h=1 / 10$.

Hence, $f$ satisfies the kinetic equation (280).

Suppose $t \in[0,+\infty$ [ fixed, one has

$$
\begin{aligned}
& \int_{\Omega \times(0,+\infty)} f(t, x, v) \mathrm{d} x \mathrm{~d} v=\int_{(0,1) \times(0,1)} \\
& {\left[\bar{x}+\frac{v^{3}}{6}+\frac{11}{12 \sqrt{\pi(4 t+1)}} e^{-\left(v^{2} / 4 t+1\right)}\right] \mathrm{d} \bar{x} \mathrm{~d} v} \\
& +\int_{(0,1) \times[1,+\infty)}\left[\frac{11}{12 \sqrt{\pi(4 t+1)}} e^{-\left(v^{2} / 4 t+1\right)}\right] \mathrm{d} \bar{x} \mathrm{~d} v \\
& =\int_{(0,1)} \bar{x} \mathrm{~d} \bar{x}+\int_{(0,1)} \frac{v^{3}}{6} \mathrm{~d} v+\int_{(0,+\infty)}\left[\frac{11}{12 \sqrt{\pi(4 t+1)}} e^{-\left(v^{2} / 4 t+1\right)}\right] \mathrm{d} v,
\end{aligned}
$$

with

$$
\begin{array}{r}
\int_{(0,1)} \bar{x} \mathrm{~d} \bar{x}=\frac{1}{2}, \\
\int_{(0,1)} \frac{v^{3}}{6} \mathrm{~d} v=\frac{1}{24}, \\
\int_{(0,+\infty)}\left[\frac{11}{12 \sqrt{\pi(4 t+1)}} e^{-\left(v^{2} / 4 t+1\right)}\right] \mathrm{d} v=\frac{11}{24} .
\end{array}
$$

Consequently, $\int_{\Omega \times(0,+\infty)} f(t, x, v) \mathrm{d} x \mathrm{~d} v=1$ for all $t \geq 0$.

5.3. The Figures. Here, we perform the first-order approximation $f_{1}$ of the solution of (278) using the algorithm in (261). We limit our self to the first-order approximation just for the sake of simplicity and to avoid very complex and cumbersome calculations. Numerical computation carried out for $h \in\{1 / 10,1 / 50,1 / 100\}$ and the graphical representations of both the analytical solution $f$ and the approximate solution $f_{1}$ with Scilab software shows a coherence between the approximate solution and the analytical solution.

Moreover, an error tables obtained for some values of the time $t$ in the interval $[h, 2 h]$ for different values of the step $h$ are presented below.

By a simple computation, the expression of $f_{1}^{h}$ appears as follows:

$$
\left\{\begin{array}{l}
f_{1}^{h}(x, v)=\bar{x}+\frac{v^{3}}{6}+\frac{11 h v^{2}}{3 \sqrt{\pi}} e^{-v^{2}}+\frac{11(1-2 h)}{12 \sqrt{\pi}} e^{-v^{2}}, \quad \text { is }(\bar{x}, v) \in(0,1) \times(0,1), \\
f_{1}^{h}(x, v)=\frac{11 h v^{2}}{3 \sqrt{\pi}} e^{-v^{2}}+\frac{11(1-2 h)}{12 \sqrt{\pi}} e^{-v^{2}}, \quad \text { is }(\bar{x}, v) \in(0,1) \times[1,+\infty) .
\end{array}\right.
$$

For some values of $h$, we draw the figures of both the analytical solution and the numerical solution (approximate solution). We notice that Figure 1 represents an approximation of Figure 2 when $h=(1 / 10)$, Figure 3 represents an approximation of Figure 4 when $h=(1 / 50)$, and Figure 5 is an approximation of Figure 6 when $h=(1 / 100)$.

Errors progression is in terms of the $L^{\infty}$ and the $L^{2}$ norms between the numerical solution $f_{1}^{h}$ and the analytical 


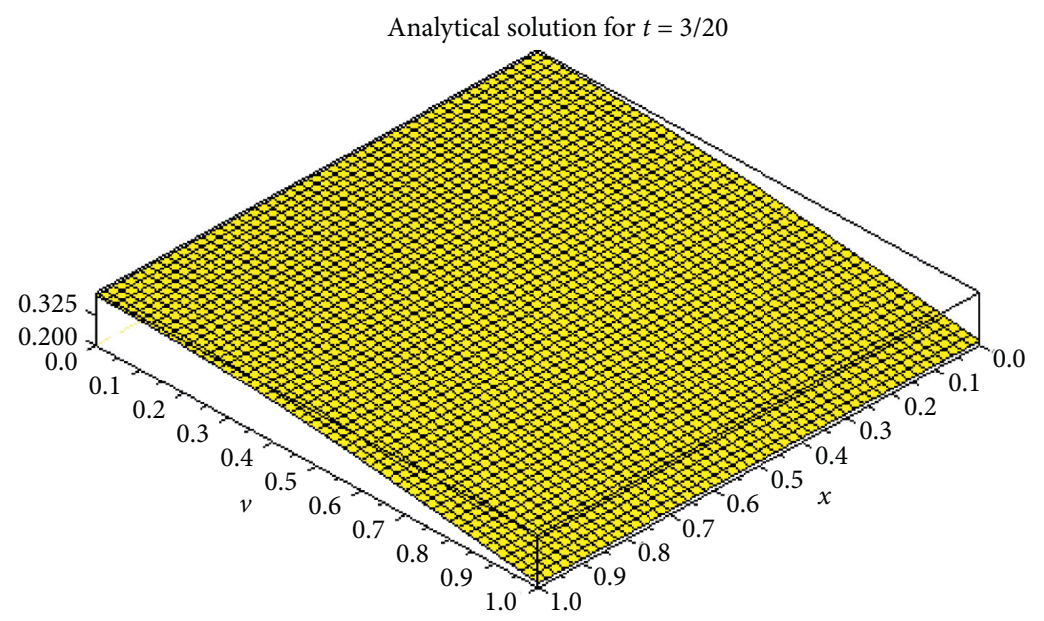

FIgURE 2: Analytical solution $f(3 / 20, x, v)$ for $h=(1 / 10)$ and $t=3 / 20 \in[1 / 10,2 / 10)$.

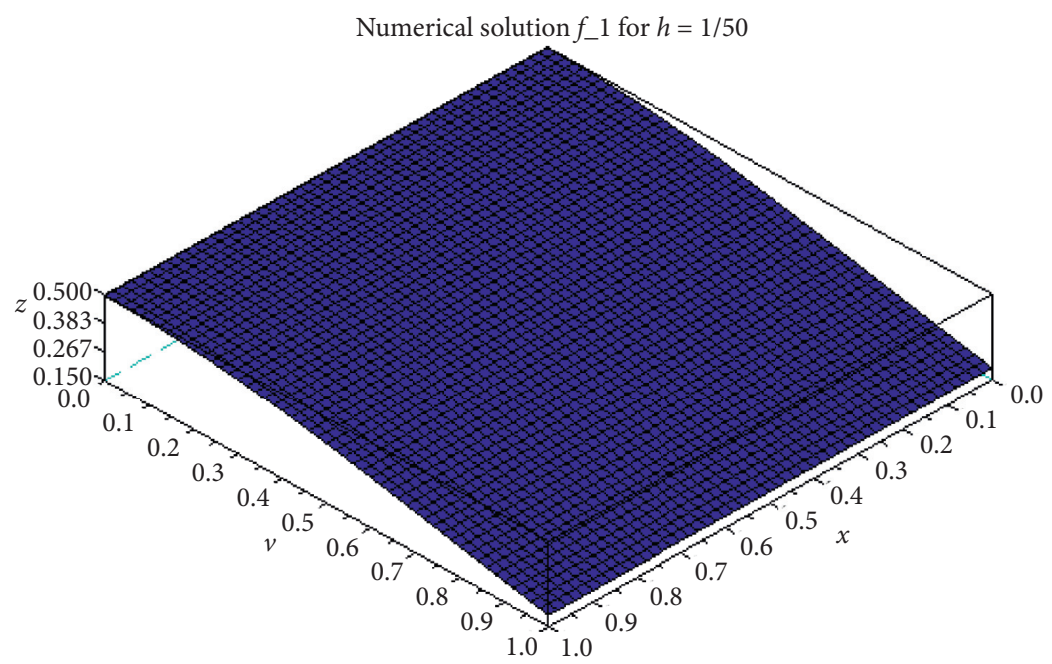

FIgURE 3: Numerical solution $f_{1}^{1 / 50}(x, v)$ for $h=1 / 50$.

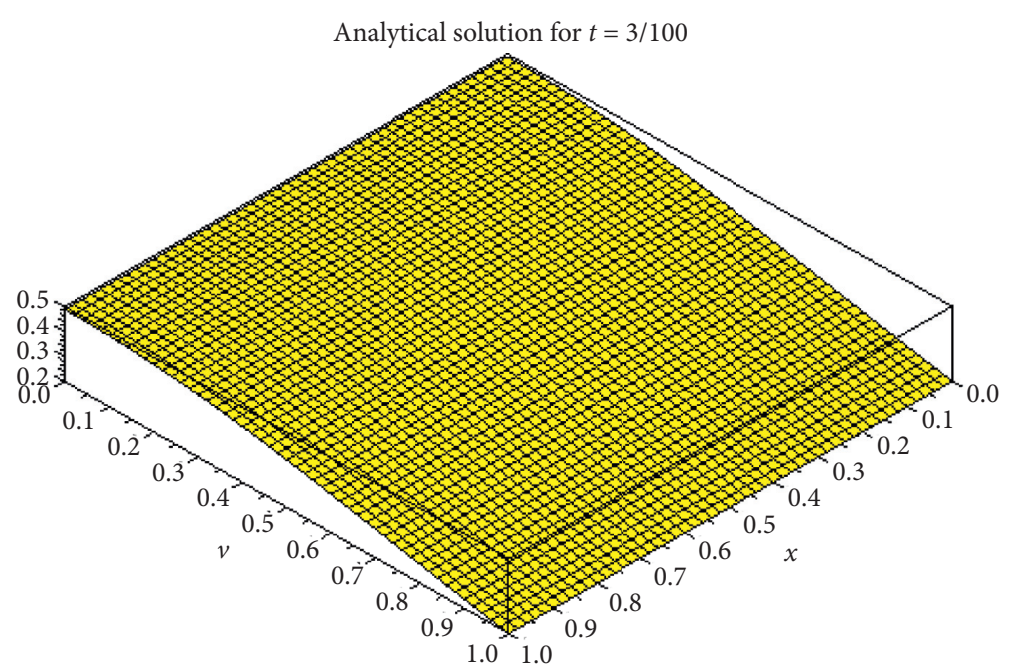

Figure 4: Analytical solution $f(3 / 100, x, v)$ for $h=1 / 50$ and $t=3 / 100 \in[1 / 50,1 / 25)$. 


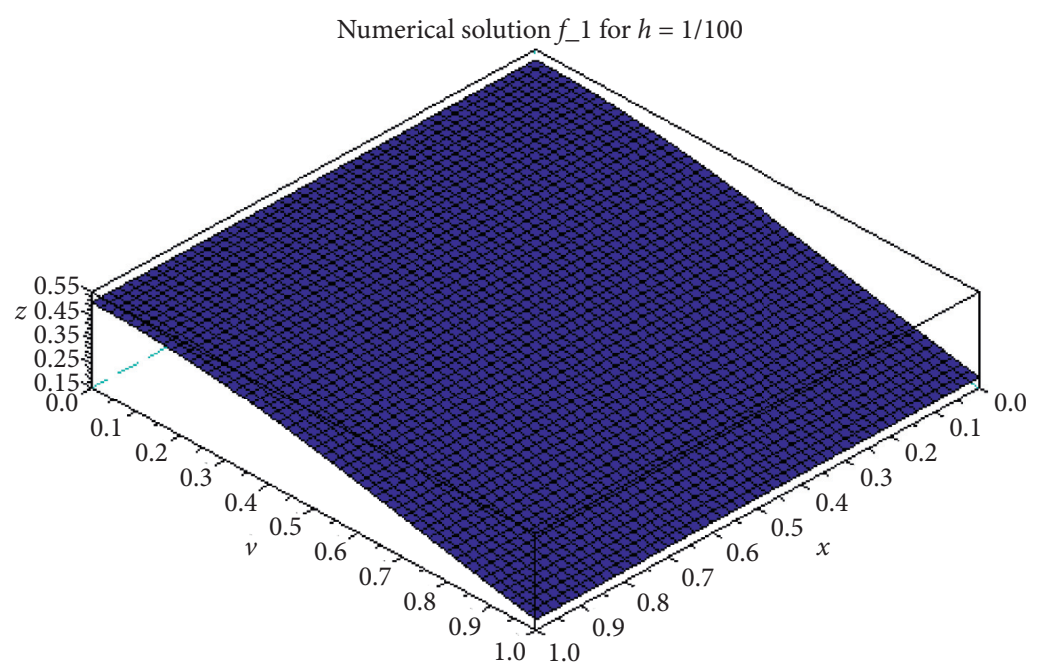

FIgURE 5: Numerical solution $f_{1}^{1 / 100}(x, v)$ for $h=1 / 100$.

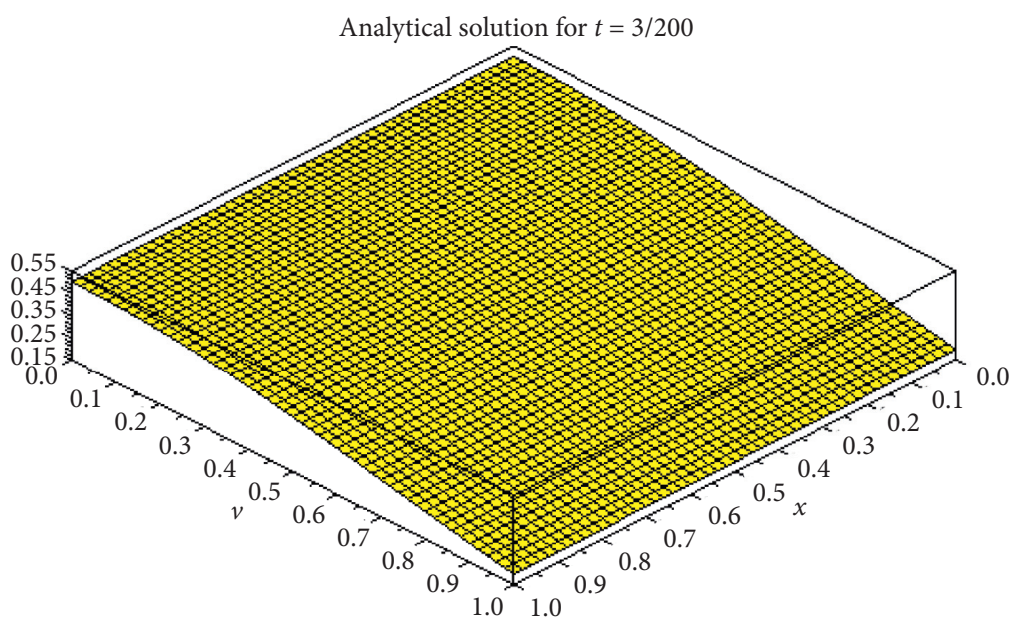

FIgURE 6: Analytical solution $f(3 / 200, x, v)$ for $h=1 / 100$ and $t=3 / 200 \in[1 / 100,1 / 50)$.

TABLE 1: Errors' progression.

\begin{tabular}{lccccc}
\hline$h$ & $1 / 10$ & $1 / 50$ & $1 / 100$ & $1 / 500$ & $1 / 1000$ \\
$\delta t$ & $3 / 20$ & $3 / 100$ & $3 / 200$ & $3 / 1000$ & $3 / 2000$ \\
$\left\|f-f_{1}^{h}\right\|_{L^{2}}$ & 0.03422791 & 0.03422791 & 0.002478 & 0.001420191 & 0.00027920 \\
$\left\|f-f_{1}^{h}\right\|_{\max }$ & 0.0145619 & 0.078035 & 0.0045067 & 0.0010067 & 0.0008798 \\
\hline
\end{tabular}

solution $f$. One can actually notice the convergence of our method in the $L^{2}$ and in $L^{\infty}$ norms when $h$ is decreasing to 0 , which is explained as follows (Table 1).

\section{Appendix}

Here, we give the regularity result necessary in the proof of Theorem 4.

Lemma 16. Assume that $f_{0}, c$, and $G$ satisfy, respectively, $\left(H_{f_{0}}\right),\left(H_{c}\right)$, and $\left(H_{G}\right)$, supp $f_{0}(x,.) \subset B_{R}$, for all $x \in \Omega$ and $\nabla c^{*}(0)=0$. Then, $\operatorname{supp} f_{k}^{h}(x,.) \subset B_{R}$, for all $x \in \Omega$ and for all $k \in \mathbb{N}^{*}$.
Moreover, $D^{2} c^{*}\left(\nabla_{v} G^{\prime}\left(f_{k}^{h}\right)\right) \circ D_{v}^{2} G^{\prime}\left(f_{k}^{h}\right) \in L^{\infty}\left(\Omega \times B_{R}\right)$ and

$$
\left\|h D^{2} c^{*}\left(\nabla_{v} G^{\prime}\left(f_{k}^{h}\right)\right) \circ D_{v}^{2} G^{\prime}\left(f_{k}^{h}\right)\right\|_{L^{\infty}\left(\Omega \times B_{R}\right)} \leq 1+\frac{M}{N} .
$$

Proof. Since supp $f_{0}(x,.) \subset B_{R}$, then $f_{0}\left(x, B_{R}^{c}\right)=0$, where $B_{R}^{c}$ is the complement of $B_{R}$. In Section 2.1, we have $f_{1}(x, v)=\rho_{1}^{h}(x) F_{1}^{x}(v)$, where

$$
\rho_{1}^{h}(x)=\int_{\mathbb{R}^{d}} f_{0}(x-h v, v) \mathrm{d} v,
$$


and $F_{1}^{x}$ is the unique solution of the variational problem.

$$
\left(P_{1}^{x}\right): \inf _{F \in P_{q}\left(\mathbb{R}^{d}\right)}\left\{\frac{1}{\rho_{1}^{h}(x)} \int_{\mathbb{R}^{d}} G\left(F \rho_{1}^{h}(x)\right) \mathrm{d} v+h W_{c}^{h}\left(F, G_{1}^{x}\right)\right\},
$$

with

$$
\begin{aligned}
& G_{1}^{x}(v)=\frac{f_{0}(x-h v, v)}{\rho_{1}^{h}(x)}, \\
& W_{c}^{h}\left(F, G_{1}^{x}\right)=\inf _{T_{\#} F=G_{1}^{x}} \int_{\mathbb{R}^{d}} c\left(\frac{v-T(v)}{h}\right) F \mathrm{~d} v .
\end{aligned}
$$

Since $f_{0}\left(x, B_{R}^{c}\right)=0$, then $G_{1}^{x}\left(B_{R}^{c}\right)=0$.

The $c$-optimal map $T_{1}$ that pushes $F_{1}^{x}$ forward to $G_{1}^{x}$ satisfies then

$$
F_{1}^{x}\left(T_{1}^{-1}\left(B_{R}^{c}\right)\right)=G_{1}^{x}\left(B_{R}^{c}\right)=0 .
$$

We now use the explicit expression of $T_{1}$ (see Lemma 3):

$$
T_{1}(v)=v+h \nabla c^{*}\left(\nabla_{v} G^{\prime}\left(f_{1}\right)\right),
$$

along with (A.5) and $\nabla c^{*}(0)=0$ to get

$$
T_{1}\left(T_{1}^{-1}\left(B_{R}^{c}\right)\right)=T_{1}^{-1}\left(B_{R}^{c}\right) .
$$

This implies that $T_{1}^{-1}\left(B_{R}^{c}\right)=B_{R}^{c}$, and then, $F_{1}^{x}\left(B_{R}^{c}\right)=0$. Thus, we deduce that supp $F_{1}^{x} \subset B_{R}$.

Since $f_{1}(x, v)=\rho_{1}^{h}(x) F_{1}^{x}(v)$, we obtain supp $f_{1}(x,.) \subset B_{R}$, for all $x \in \Omega$. Finally, we obtain by induction supp $f_{k}^{h}(x,.) \subset B_{R}$, for all $x \in \Omega$.

Since $F_{k}^{x h}$ and $G_{k}^{x h}$ have compact support and that $c$ is strictly convex, then the $c$-optimal maps $T_{k}$ that pushes $F_{k}^{x h}$ forward to $G_{k}^{x h}$ is differentiable, and we have

$$
\nabla_{v} T_{k}(v)=i d+h D^{2} c^{*}\left(\nabla_{v} G^{\prime}\left(f_{k}^{h}\right)\right) \circ D_{v}^{2} G^{\prime}\left(f_{k}^{h}\right) .
$$

Hence, $\nabla_{v} T_{k}(v)$ satisfies the Jacobian equation:

$$
\rho_{k}^{h} F_{k}^{x h}=\operatorname{det}\left(\nabla_{v} T_{k}(v)\right) \rho_{k}^{h} G_{k}^{x h} .
$$

Since $N \leq f_{0} \leq M$, the maximum principle gives $N \leq f_{k}^{h} \leq M$. Then, we deduce that

$$
\frac{N}{M} \leq \operatorname{det}\left(\nabla_{v} T_{k}(v)\right) \leq \frac{M}{N}
$$

Note that $\nabla_{v} T_{k}$ is diagonalizable and has positive eigenvalues (see [1]). Then, we deduce that $\nabla_{v} T_{k}(v) \in L^{\infty}(\Omega \times$ $\left.B_{R}\right)$ and $\left\|\nabla_{v} T_{k}(v)\right\|_{L^{\infty}\left(\Omega \times B_{R}\right)} \leq(M / N)$. And by using (A.8), we obtain

$$
\left\|h D^{2} c^{*}\left(\nabla_{v} G^{\prime}\left(f_{k}^{h}\right)\right) \circ D_{v}^{2} G^{\prime}\left(f_{k}^{h}\right)\right\|_{L^{\infty}\left(\Omega \times B_{R}\right)} \leq 1+\frac{M}{N} .
$$

\section{Data Availability}

There are no data underlying the findings in this paper to be shared.

\section{Conflicts of Interest}

The authors declare that they have no conflicts of interest.

\section{Acknowledgments}

This subject was suggested to us by the late Professor and friend Martial Agueh, University of Victoria, Canada. The authors pay their heartfelt respect to his memory. This work was supported by IMSP under the CEA-SMA project grant.

\section{References}

[1] K. El Miloudi and E. Aziz, "A Multiview formal Model of use Case Diagrams using $\mathrm{Z}$ notation: towards improving functional Requirements quality," Journal of Engineering, vol. 2018, Article ID 6854920, 9 pages, 2018.

[2] M. Agueh, "Local Existence of weak solutions to Kinetic models of granular media," Archive for Rational Mechanics and Analysis, vol. 221, no. 2, pp. 917-959, 2016.

[3] M. Agueh and G. Carlier, "Generalized solutions of Kinetic granular media equation by a gradient flow approach," Calculus of Variations and Partial Differential Equations, vol. 55, no. 2, p. 37, 2016.

[4] A. A. Arsen'ev, "Existence in the large of weak solution of Vlasov's system of equations," Zhurnal Vychistel'noi Matematiki i Matematicheskoi Fiziki, vol. 15, no. 1, pp. 136-147, 1975.

[5] E. Carlen and W. Gangbo, "Solution of a model Boltzmann equation via steepest descent in the 2-Wasserstein metric," Archive for Rational Mechanics and Analysis, vol. 172, no. 1, pp. 21-64, 2004.

[6] R. Jordan, D. Kinderlehrer, and F. Otto, "The variational formulation of the Fokker--Planck equation," SIAM Journal on Mathematical Analysis, vol. 29, no. 1, pp. 1-17, 1998.

[7] H. Zhan, "The boundary value condition for an evolutionary $p(x)$-Laplacian equation," Boundary Value Problems, vol. 2015112 pages, 2015.

[8] L. Ambrosio, N. Gigli, and G. Savaré, "Gradient flow in metric spaces and in the space of probability measures," in Lectures in Mathematics, Birkhauser, Basel, Switzerland, 2005.

[9] H. Brezis, Functional Analysis, Springer, New York, NY, USA, 1987.

[10] J. Geiser, "Iterative operator-splitting methods for nonlinear differential equations and applications of deposition processes," Numerical Methods for Partial Differential Equations, vol. 27 , no. 5, pp. 1026-1054, 2011. 